\section{Keywords: Tank Farm \\ Characterization \\ Sludge \\ ESP \\ DWPF}

Retention Time: Permanent

\title{
Results of the Comparison Analyses of the Two Tank 40H Sludge Batch 2 Qualification Samples
}

\author{
R. F. Swingle \\ N. E. Bibler \\ C. J. Coleman \\ T. L. Fellinger \\ T. B. Edwards
}

Publication Date: November 26, 2001 
This document was prepared in conjunction with work accomplished under Contract No. DE-AC09-96SR18500 with the U.S. Department of Energy.

\section{DISCLAIMER}

This report was prepared as an account of work sponsored by an agency of the United States Government. Neither the United States Government nor any agency thereof, nor any of their employees, makes any warranty, express or implied, or assumes any legal liability or responsibility for the accuracy, completeness, or usefulness of any information, apparatus, product or process disclosed, or represents that its use would not infringe privately owned rights. Reference herein to any specific commercial product, process or service by trade name, trademark, manufacturer, or otherwise does not necessarily constitute or imply its endorsement, recommendation, or favoring by the United States Government or any agency thereof. The views and opinions of authors expressed herein do not necessarily state or reflect those of the United States Government or any agency thereof.

This report has been reproduced directly from the best available copy.

Available for sale to the public, in paper, from: U.S. Department of Commerce, National Technical Information Service, 5285 Port Royal Road, Springfield, VA 22161

phone: (800) 553-6847

fax: (703) 605-6900

email: orders@ntis.fedworld.gov

online ordering: http://www.ntis.gov/support/index.html

Available electronically at http://www.doe.gov/bridge

Available for a processing fee to U.S. Department of Energy and its contractors, in paper, from: U.S. Department of Energy, Office of Scientific and Technical Information, P.O. Box 62, Oak Ridge, TN 37831-0062

phone: (865)576-8401

fax: $(\mathbf{8 6 5}) 576-5728$

email: reportseadonis.osti.gov 


\section{SUMMARY}

Two sets of six samples each of Sludge Batch 2 material were pulled from Tank $40 \mathrm{H}$ after completion of the transfer of the contents of Tank 8F to Tank $40 \mathrm{H}$. At the request of Defense Waste Processing Facility (DWPF) personnel, these two sets of samples were analyzed to verify that there were no significant differences between the two sets due to differences in slurry pump operation time prior to pulling the samples.

The results of those analyses indicate that the two samples are within $1.2 \%$ of each other for weight percent total solids in the slurry and weight percent dissolved solids in the supernate. Elemental analyses of the total slurry by Inductively Coupled Plasma-Mass Spectrometry (ICP-ES) gave differences of less than 2\%. Analyses of selected fission product isotopes and actinides produced differences of generally less than 4\%, though a couple of isotopes showed differences of up to $6 \%$. With the exception of a few of the elemental analyses, whose differences were less than $1.5 \%$, and Cs-137, whose concentration difference between the two samples was found to be about $3 \%$, none of these differences was found to be statistically significant. This differences were compared to a criteria of $<5 \%$ difference.

The differences in the $\mathrm{Na}$ and $\mathrm{Al}$ concentrations in the supernate were found to be $6-8 \mathrm{wt} \%$. However, because of the analytical variability, these differences were not considered to be statistically significant.

Based on these results, the samples are considered to be essentially the same.

\section{INTRODUCTION}

A task technical and quality assurance plan was previously prepared for the characterization and washing of the Sludge Batch 2 Qualification Sample. ${ }^{1}$ The twelve Tank 40H samples needed for this work were pulled in two groups of six samples each. The first group of six samples was pulled on June 29, 2001 after running the slurry pumps continuously for 32 hours. The second group of six samples was pulled on July 5, 2001, after running the slurry pumps continuously for 60 hours. All previous sludge batch qualification samples were pulled after running the slurry pumps for at least 48 hours, though not always continuously. Concerns were raised about whether the first set six samples subjected to only 32 hours of slurry pump operation was representative of the Tank $40 \mathrm{H}$ contents as a whole. DWPF personnel requested separate analysis and comparison of the two sets of samples to verify that both sets are representative of the tank contents. ${ }^{2}$ The intent of this comparison was to ensure that Tank 8 sludge was thoroughly mixed with Tank 40 sludge by comparison of the major species and that the sludge was properly suspended in the tank by comparison of the sludge and supernate ratios between the two sample sets. Once aliquots were pulled from the two composite samples, the two sets of sludge were combined and the washing of the sludge for qualification proceeded in parallel with the verification of the samples. ${ }^{1}$ A final decision to proceed to completion of the Sludge Batch 2 qualification using the current samples or to pull new samples was made after completion of the work documented herein.

\section{DISCUSSION}

\section{Task Description}

Twelve 200-mL samples of sludge slurry from Tank $40 \mathrm{H}$ (containing blended sludge from Tanks $40 \mathrm{H}$ and $8 \mathrm{~F}$ ) were transported to the SRTC Shielded Cells Facility in two sets of six. Each set of six samples was composited. The following operations were conducted on these samples. 
1. An aliquot of each composite slurry was filtered. The filtrate from this aliquot was analyzed for density and weight percent dissolved solids. The dried solids from the weight percent solids measurements were then redissolved in aqua regia and submitted to Analytical Development Section (ADS) for analysis by Inductively Coupled Plasma - Emission Spectrometry (ICP-ES) for Ag, Fe, Al, $\mathrm{Na}, \mathrm{Mg}$ and $\mathrm{Mn}$, by Inductively Coupled Plasma - Mass Spectrometry (ICP-MS) for Rh, Ru, Pd, and U and by gamma scan for detectable gamma emitting radionuclides such as Cs-137 and Am-241. Seven replicates of each analysis were required for each sample to verify whether the samples differed statistically by $5 \%$ or more. Ten replicates of each analysis, except density were run for each composite sample to allow for lost samples or bad results.

2. The density was measured on a sample of each composite slurry. Since density measurements are not required by the TTR, only 3 replicate measurements were made for each slurry. Ten aliquots of each slurry were dried to constant weight to determine the weight percent total solids. The remaining dried solids from each weight percent solids determination was dissolved in aqua regia. In addition, four aliquots of analytical reference glass (ARG) were dissolved in aqua regia. The 26 dissolved solids samples (twenty samples, four ARG standards and two blanks) were submitted to ADS for ICP-ES, ICP-MS and gamma scan for detectable gamma emitting radionuclides such as $\mathrm{Cs}-137$ and Am-241. These samples were run in a specified order to ensure systematic errors did not cause a false difference to appear.

3. The results of these analyses for each of the two composite samples were compared to check for any statistical differences in composition. Based on those results, a decision was made to proceed with the current samples instead of pulling a new sample for the glass qualification run.

\section{Results}

The measurements generated by the Analytical Development Section (ADS) and Shielded Cells Operations (SCO) are presented in this section. Table A1 in the Appendix provides the weight percent (wt $\%$ ) total solids for each of 20 samples. Table A2 in the Appendix provides the analyses of elemental concentrations (in wt $\%$ wet solids) for 20 samples by ICP-ES. These analyses were completed over 2 calibrations of the ICP. The calibration is provided as a column in this table. Also, samples of ARG-1 and a blank were included in each analytical block along with the composite samples for analysis by ICP-ES. The results from these standards also are provided in Table A2.

The last column of Table A2 contains values for the ratio of the iron to sodium measurements for each sample and standard. This ratio provides a quantity for comparison across the study samples that is insensitive to some of variations that might affect individual elemental concentration measurements such as variations in recorded weights.

Table A3 in the Appendix provides ICP-MS elemental concentrations in weight percent of slurry for selected fission products and actinides by mass number for the Tank 40 samples along with ARG-1 and blank samples. Table A4 in the Appendix provides measurements (via Gamma scan) of the radioactivity of cobalt-60 and cesium137 in dpm per gram of slurry for 10 samples of each of the Tank 40 composites. Measurements from samples of ARG-1 and blanks are also presented in this table, and as to be expected, the results from these samples were all below the detection limits of the analytical process.

Analyses were also conducted on supernate samples of each of the Tank 40 composite samples. Table A5 provides weight percent $(\mathrm{wt} \%$ ) solids for the supernate samples, and Table A6 provides elemental concentrations for $\mathrm{Al}$ and $\mathrm{Na}$ in grams of analyte per gram of filtered supernate. 


\section{STATISTICAL ANALYSES}

The information presented in the Tables A1 through A6 provides the basis for the statistical comparisons of the two composite samples of Tank 40. The data in each of these tables is reviewed in turn. The statistical comparisons for these measurements were conducted using JMP® Version 4.0, a commercially available statistical software package from SAS Institute, $\operatorname{Inc}^{3}$.

\section{Weight Percent Solids of the Slurry}

Plots of the slurry weight percent total solids data in Table A1 by type of composite are provided in Exhibit A1 of the Appendix. There is no indication of outliers in these two datasets; nor is there an indication of different variances for the two datasets. The JMP results show a statistically significant difference (at the $5 \%$ significance level) of $0.3272 \mathrm{wt} \%$ between the means of the two types of composites. The 32-hr composite average wt $\%$ solids value $(26.35 \mathrm{wt} \%)$ is $1.2 \%$ below the 60 -hr average $(26.68 \mathrm{wt} \%)$. Although statistically significant, this $1.2 \%$ difference is seen as being of little practical concern.

\section{Elemental Analyses of the Slurry from ICP-ES}

Key elemental analyses were conducted on slurry samples from each of the composites using an aqua regia dissolution method and ICP-ES. As discussed above, these results are provided in Table A2 in the Appendix. Two calibrations of the ICP-ES were required to complete these analyses. Plots of these elemental concentrations (as wet wt\%'s of slurry) by type of composite and calibration are provided in Exhibit A2 in the Appendix. These plots indicate a significant effect due to ICP-ES calibration for many of the results. Also, there appear to be potential outliers in these data. The initial statistical analyses conducted on these results use all of the data. To make sure that the potential outliers are not driving the conclusions, an analysis with potential outliers removed is also conducted.

Measurements of ARG-1 and blanks are also presented in Table A2. Table 1 provides a summary of the information for ARG-1. The calibration of the ICP-ES again had an impact on the resulting elemental concentration measurements. Also in this table, are the reference values for the elemental concentrations and percent differences between the measured and reference values.

Table 1. ARG-1 Elemental Concentrations (as wt\%'s) by ICP-ES Calibration Blocks

\begin{tabular}{|c|c|c|c|c|c|c|c|c|c|c|}
\hline LIMS \# & Composite Type & Calibration & Al & $\mathbf{C a}$ & $\mathbf{F e}$ & $\mathrm{Mg}$ & Mn & $\mathbf{N a}$ & $\mathbf{U}$ & $\mathrm{Fe} / \mathrm{Na}$ \\
\hline 300166375 & ARG-1/1 & 1 & 2.51 & & 10.73 & 0.57 & 1.61 & 8.65 & 0.29 & 1.24 \\
\hline 300166384 & ARG-1/1 & 1 & 2.50 & & 10.63 & 0.56 & 1.55 & 8.54 & 0.29 & 1.25 \\
\hline 300166392 & ARG-1/2 & 2 & 2.36 & & 10.12 & 0.54 & 1.54 & 8.50 & 0.00 & 1.19 \\
\hline \multirow[t]{4}{*}{300166396} & ARG-1/2 & 2 & 2.37 & & 10.01 & 0.54 & 1.51 & 8.51 & 0.00 & 1.18 \\
\hline & & Average & 2.43 & & 10.37 & 0.55 & 1.55 & 8.55 & 0.14 & 1.21 \\
\hline & ARG -1 & Reference & 2.5 & & 9.79 & 0.52 & 1.46 & 8.52 & 0 & 1.15 \\
\hline & & $\%$ Difference & $-2.70 \%$ & & $6.00 \%$ & $6.00 \%$ & $6.40 \%$ & $0.30 \%$ & & $5.60 \%$ \\
\hline
\end{tabular}


Exhibit A3 in the Appendix provides the details of a statistical analysis of variance using the full set of measurements for each element of interest. This analysis investigates for both composite and calibration effects. Table 2 summarizes the results from these analyses. As seen in Table 2, there are no statistically significant (at the $5 \%$ level) differences between the means of the two composites for the elements measured using ICP-ES. The differences expressed as percentages of the 60-hr averages are all less than $1 \%$. The sensitivity of each of these statistical comparisons is explored in Exhibit A4 in the Appendix. The information in this exhibit is complemented by the last column of Table 2, which provides the percent difference between the averages from the two composites that would have been detected with a power of $90 \%$ (i.e., with a $90 \%$ probability) based upon the variation seen in the measurements and the number of measurements conducted."

Table 2. Average Elemental Concentrations(as wet wt\%'s of slurry) by Type of Composite

\begin{tabular}{|c|c|c|c|c|c|c|}
\hline Sample Type & 32 & 60 & & Percent (\%) & $\begin{array}{c}\text { Statistically } \\
\text { Significant } \\
\text { At the }\end{array}$ & $\begin{array}{c}\text { \% Difference } \\
\text { Detectable } \\
\text { With }\end{array}$ \\
\hline N Rows & 10 & 10 & Difference & Difference & $5 \%$ Level & 90\% Power \\
\hline Ag & $7.84 \mathrm{E}-03$ & $7.85 \mathrm{E}-03$ & $-1.00 \mathrm{E}-05$ & $-0.13 \%$ & No & $7.37 \%$ \\
\hline Al & 1.39 & 1.39 & $1.20 \mathrm{E}-04$ & $0.01 \%$ & No & $6.90 \%$ \\
\hline Ca & 0.498 & 0.501 & $-2.36 \mathrm{E}-03$ & $-0.47 \%$ & No & $4.92 \%$ \\
\hline $\mathbf{F e}$ & 5.14 & 5.13 & 9.37E-03 & $0.18 \%$ & No & $7.37 \%$ \\
\hline Mg & 0.414 & 0.413 & $1.14 \mathrm{E}-03$ & $0.28 \%$ & No & $7.46 \%$ \\
\hline Mn & 0.702 & 0.699 & $2.80 \mathrm{E}-03$ & $0.40 \%$ & No & $7.52 \%$ \\
\hline $\mathbf{N a}$ & 4.20 & 4.18 & 2.43E-02 & $0.58 \%$ & No & $6.38 \%$ \\
\hline $\mathbf{U}$ & 1.58 & 1.59 & $-1.12 \mathrm{E}-02$ & $-0.70 \%$ & No & $3.69 \%$ \\
\hline $\mathrm{Fe} / \mathrm{Na}$ & 1.22 & 1.23 & $-4.69 \mathrm{E}-03$ & $-0.38 \%$ & No & $2.26 \%$ \\
\hline
\end{tabular}

Exhibit A5 in the Appendix provides the details of a statistical analysis of variance using the measurements for each element of interest with potential outliers removed. The LIMS numbers of the excluded measurements are given as part of the information of the exhibit. The outliers were identified by a simple review of the plots presented in Exhibit A1. Although this is a subjective process and other ways of removing potential problem data might be used, this approach does provide some protection from drawing conclusions that are overly sensitive to only a few of the measurements from these samples. The analysis in Exhibit A5 investigates for both composite and calibration effects for the screened data. Table 3 summarizes the results from these analyses. As seen in Table 3 , a few of the analytes ( $\mathrm{Al}, \mathrm{Ca}$, and $\mathrm{Na}$ ) show a statistically significant (at the 5\% level) difference between the means of the two composites. However, each of the differences expressed as percentages of the 60-hr averages is less than $2 \%$.

The sensitivity of each of these statistical comparisons in the form of power details is explored as part of the information provided in Exhibit A5. The power of each of these comparisons to detect a 5\% difference in the two composite means is provided. The smallest power is that for $\mathrm{Ag}(89.7 \%)$, while all of the other elements show a power greater than $90 \%$. This information is complemented by the last column of Table 3, which provides the percent difference between the averages from the two composites that would have been detected with a power of $90 \%$ (i.e., with a $90 \%$ probability) based upon the variation seen and the number observations remaining in the screened data.

- These power calculations were conducted using the "Design of Experiments" platform of JMP® Version 4.0 [3]. 
WSRC-TR-2001-00565

Page 6 of 83

November 26, 2001

Table 3. Average Elemental Concentrations (as wet wt\%'s of slurry) of the Screened Measurements by Type of Composite

\begin{tabular}{|c|c|c|c|c|c|c|}
\hline Sample Type & 32 & 60 & & Percent (\%) & $\begin{array}{c}\text { Statistically } \\
\text { Significant } \\
\text { At the }\end{array}$ & $\begin{array}{c}\text { \% Difference } \\
\text { Detectable } \\
\text { With }\end{array}$ \\
\hline N Rows & 10 & 10 & Difference & Difference & $5 \%$ Level & 90\% Power \\
\hline Ag & 7.72E-03 & 7.79E-03 & $-7.00 \mathrm{E}-05$ & $-0.86 \%$ & No & $5.02 \%$ \\
\hline Al & 1.35 & 1.37 & $-1.90 \mathrm{E}-02$ & $-1.38 \%$ & Yes & $1.55 \%$ \\
\hline $\mathbf{C a}$ & 0.490 & 0.498 & $-7.26 \mathrm{E}-03$ & $-1.46 \%$ & Yes & $1.26 \%$ \\
\hline $\mathbf{F e}$ & 5.05 & 5.10 & $-4.52 \mathrm{E}-02$ & $-0.89 \%$ & No & $3.83 \%$ \\
\hline Mg & 0.403 & 0.410 & $-6.80 \mathrm{E}-03$ & $-1.66 \%$ & No & $3.25 \%$ \\
\hline Mn & 0.684 & 0.695 & $-1.15 \mathrm{E}-02$ & $-1.66 \%$ & No & $3.28 \%$ \\
\hline $\mathbf{N a}$ & 4.11 & 4.15 & $-4.12 \mathrm{E}-02$ & $-0.99 \%$ & Yes & $1.23 \%$ \\
\hline $\mathbf{U}$ & 1.57 & 1.58 & $-7.00 \mathrm{E}-03$ & $-0.44 \%$ & No & $3.31 \%$ \\
\hline $\mathrm{Fe} / \mathrm{Na}$ & 1.22 & 1.23 & $-8.59 \mathrm{E}-03$ & $-0.70 \%$ & No & $2.10 \%$ \\
\hline
\end{tabular}

Even though Al, Ca, and Na show a statistically significant difference between the two Tanks 40 composites, these differences for the ICP-ES measurements are all less than $2 \%$ and deemed to be of no practical significance. Thus, the overall conclusions for the ICP-ES data are that there are no differences of practical concern between the two composites.

\section{Elemental Analyses from ICP-MS}

Analyses of selected fission products and actinides were conducted on samples from each of the composite using an aqua regia dissolution method and ICP-MS. As discussed above, these results are provided by mass number in Table A3 of the Appendix. Two calibrations of the ICP-MS were required to complete these analyses. Samples with LIMS numbers 300166387 and 300177388 were measured at the end of the first calibration block and again at the beginning of the second calibration block. The values for these samples over the two calibrations in Table A3 suggest the potential for calibration effects, as was seen in the ICP-ES results. Only the measurements for these two samples generated during the second ICP-MS calibration are included in the analyses that follow.

Plots of these concentrations (as micrograms per gram of slurry) for each mass number of interest by type of composite and ICP-MS calibration are provided in Exhibit A6 in the Appendix. These plots indicate a significant effect due to ICP-MS calibration for many of the results. Also, there appear to be potential outliers in these data. The initial statistical analyses conducted on these results use all of the data. To make sure that the potential outliers are not driving the conclusions, an analysis with potential outliers removed is also conducted.

Exhibit A7 in the Appendix provides the details of a statistical analysis of variance using the full set of measurements for each mass number of interest. This analysis investigates for both composite and calibration effects. Table 4 summarizes the results from these analyses. As seen in Table 4, there are no statistically significant differences (at the 5\% level) between the means of the two composites for the selected fission products and actinides measured using ICP-MS. The differences expressed as percentages of the 60-hr averages are all less than 7\%. The sensitivity of each of these statistical comparisons is explored in Exhibit A8 in the Appendix. The information in this exhibit is complemented by the last column of Table 4, which provides the percent difference between the averages from the two composites that would have been detected with a power of $90 \%$ (i.e., with a $90 \%$ probability) based upon the variation seen in the data and the number of measurements. 
WSRC-TR-2001-00565

Revision 0

Page 7 of 83

November 26, 2001

Table 4. Average Concentrations (as micrograms/gram of slurry)for Each Mass Number of Interest by Type Composite

\begin{tabular}{|lcccccc|}
\hline & & & & & Statistically & \% Difference \\
Significant & Detectable \\
Sample Type & $\mathbf{3 2}$ & $\mathbf{6 0}$ & & Percent (\%) & $\begin{array}{c}\text { At the } \\
\text { With }\end{array}$ \\
N Rows & $\mathbf{1 0}$ & $\mathbf{1 0}$ & Difference & $\begin{array}{c}\text { Difference } \\
\mathbf{5 \%} \text { Level }\end{array}$ & $\mathbf{9 0 \% \text { Power }}$ \\
Tc-99 & 1.75 & 1.69 & 0.051 & $3.01 \%$ & No & $14.13 \%$ \\
Ru-101 & 26.6 & 26.0 & 0.614 & $2.36 \%$ & No & $4.66 \%$ \\
Ru-102 & 25.2 & 24.8 & 0.330 & $1.33 \%$ & No & $5.31 \%$ \\
Rh-103 & 16.8 & 16.5 & 0.270 & $1.63 \%$ & No & $4.84 \%$ \\
Ru-104 & 16.9 & 16.4 & 0.480 & $2.93 \%$ & No & $4.98 \%$ \\
Pd-105 & 1.22 & 1.30 & -0.081 & $-6.23 \%$ & No & $13.00 \%$ \\
Ag-107 & 10.2 & 9.836 & 0.319 & $3.25 \%$ & No & $9.16 \%$ \\
Ag-109 & 9.83 & 9.48 & 0.356 & $3.76 \%$ & No & $10.73 \%$ \\
U-235 & 55.3 & 54.6 & 0.711 & $1.30 \%$ & No & $4.77 \%$ \\
U-238 & $1.35 \mathrm{E}+04$ & $1.34 \mathrm{E}+04$ & 40.1 & $0.30 \%$ & No & $6.71 \%$ \\
Pu-239 & 21.8 & 20.9 & 0.919 & $4.40 \%$ & No & $7.49 \%$ \\
\hline
\end{tabular}

Exhibit A9 in the Appendix provides the details of a statistical analysis of variance using the measurements for each element of interest with potential outliers removed. The LIMS numbers of the excluded measurements are given as part of the information of the exhibit. The outliers were identified by a simple review of the plots presented in Exhibit A6. Although this is a subjective process and other ways of removing potential problem data might be used, this approach does provide some protection from drawing conclusions that are overly sensitive to only a few of the measurements from these samples. The analysis in Exhibit A9 investigates for both composite and calibration effects for the screened data. Table 5 summarizes the results from these analyses. As seen in Table 5 , none of these analytes show a statistically significant difference (at the $5 \%$ level) between the means of the two composites. In addition, all of the differences expressed as percentages of the 60 -hr averages are less than $6 \%$.

The sensitivity of each of these statistical comparisons in the form of a power calculation is explored by the last column of Table 5, which provides the percent difference between the averages from the two composites that would have been detected with a power of $90 \%$ (i.e., with a $90 \%$ probability) based upon the variation seen and the number of observations remaining in the screened ICP-MS data. For about half of these analytes, the sensitivity attained is comparable to the detection of a 5\% difference with a $90 \%$ probability that was targeted for most of the comparisons outlined in the TT\&QA plan. The worst sensitivity is that attained for Pd-105 (14.14\%). As suggested in the TT\&QA plan, the sensitivity of the ICP-MS analyses was considered to be more uncertain than the sensitivity of the ICP-ES analyses because the species analyzed by ICP-MS were closer to the instrument detection limit. 
WSRC-TR-2001-00565

Revision 0

Page 8 of 83

November 26, 2001

Table 5. Average Mass Concentrations (in micrograms per gram of slurry) of the Screened Measurements by Type Composite

\begin{tabular}{|c|c|c|c|c|c|c|}
\hline Sample Type & 32 & 60 & & Percent $(\%)$ & $\begin{array}{c}\text { Statistically } \\
\text { Significant } \\
\text { At the }\end{array}$ & $\begin{array}{c}\text { \% Difference } \\
\text { Detectable } \\
\text { With }\end{array}$ \\
\hline N Rows & 10 & 10 & Difference & Difference & $5 \%$ Level & 90\% Power \\
\hline Tc-99 & 1.79 & 1.69 & 0.101 & $5.94 \%$ & No & $10.5 \%$ \\
\hline Ru-101 & 26.6 & 26.3 & 0.321 & $1.22 \%$ & No & $3.64 \%$ \\
\hline Ru-102 & 25.2 & 25.1 & 0.046 & $0.18 \%$ & No & $3.74 \%$ \\
\hline Rh-103 & 16.8 & 16.7 & 0.089 & $0.53 \%$ & No & $3.19 \%$ \\
\hline Ru-104 & 16.9 & 16.5 & 0.324 & $1.96 \%$ & No & $3.94 \%$ \\
\hline Pd-105 & 1.22 & 1.28 & -0.055 & $-4.35 \%$ & No & $14.1 \%$ \\
\hline Ag-107 & 10.0 & 9.84 & 0.209 & $2.12 \%$ & No & $8.82 \%$ \\
\hline Ag-109 & 9.71 & 9.48 & 0.230 & $2.43 \%$ & No & $10.3 \%$ \\
\hline U-235 & 55.3 & 55.2 & 0.077 & $0.14 \%$ & No & $2.72 \%$ \\
\hline U-238 & $1.32 \mathrm{E}+04$ & $1.34 \mathrm{E}+04$ & $-2.21 E+02$ & $-1.65 \%$ & No & $5.52 \%$ \\
\hline Pu-239 & 21.8 & 21.1 & 0.719 & $3.41 \%$ & No & $7.08 \%$ \\
\hline
\end{tabular}

\section{Radioactivity Measured by Gamma Scan}

Cobalt and cesium radioactivity (in dpm per gram of slurry) was measured on samples from each of the composite by gamma scan. As discussed above, these results are provided in Table A4 in the Appendix. Two calibrations of the instrumentation were required to complete these analyses. Plots of these activities (in dpm per gram of slurry) by type composite and calibration are provided in Exhibit A10 in the Appendix. These plots indicate little to no effect due to instrument calibration for these results. Once again, statistical analyses will be conducted on both the full set and the screened data to make sure that any potential outliers are not driving the conclusions.

Exhibit A11 in the Appendix provides the details of a statistical analysis of variance using the full set of cobalt and cesium radioactivity measurements. This analysis investigates for both composite and calibration effects. Table 6 summarizes the results from these analyses. As seen in Table 6, there are no statistically significant (at the 5\% level) differences between the means of the two composites for these measurements. The differences expressed as percentages of the 60-hr averages are all less than $4 \%$. The sensitivity of each of these statistical comparisons is explored in Exhibit A12 in the Appendix. The information in this exhibit is complemented by the last column of Table 6, which provides the percent difference between the averages from the two composites that would have been detected with a power of $90 \%$ (i.e., with a $90 \%$ probability) based upon the variation seen in the data and the number of measurements.

Table 6. Average Cobalt and Cesium Radioactivity (in dpm per gram of slurry) by Type Composite

\begin{tabular}{|lcccccc|}
\hline & & & & & $\begin{array}{c}\text { Statistically } \\
\text { Significant }\end{array}$ & $\begin{array}{c}\text { \% Difference } \\
\text { Detectable }\end{array}$ \\
Sample Type & $\mathbf{3 2}$ & $\mathbf{6 0}$ & & Percent (\%) & $\begin{array}{c}\text { At the } \\
\text { With }\end{array}$ \\
$\mathbf{N}$ Rows & $\mathbf{1 0}$ & $\mathbf{1 0}$ & Difference & Difference & $\mathbf{5 \%}$ Level & $\mathbf{9 0 \%}$ Power \\
${ }^{\mathbf{6}} \mathbf{C o}$ & $2.70 \mathrm{E}+06$ & $2.62 \mathrm{E}+06$ & $8.10 \mathrm{E}+04$ & $3.09 \%$ & No & $9.26 \%$ \\
${ }^{\mathbf{1 3 7}} \mathbf{C s}$ & $1.87 \mathrm{E}+08$ & $1.83 \mathrm{E}+08$ & $4.50 \mathrm{E}+06$ & $2.46 \%$ & No & $7.23 \%$ \\
\hline
\end{tabular}


Exhibit A13 in the Appendix provides the details of a statistical analysis of variance using the radioactivity measurements for each element of interest with potential outliers removed. The LIMS numbers of the excluded measurements are given as part of the information of the exhibit. The outliers were identified by a simple review the plots presented in Exhibit A10. Although this is a subjective process and other ways of removing potential problem data might be used, this approach does provide some protection from drawing conclusions that are overly sensitive to only a few of the measurements from these samples. The analysis in Exhibit A13 investigates for both composite and calibration effects for the screened data. Table 7 summarizes the results from these analyses. As seen in Table 7, only Cs-137 shows a statistically significant difference (at the 5\% level) between the means of the two composites for these radioactivity measurements. The Cs-137 difference is only $3.28 \%$, and the Co-60 difference is only $1.76 \%$.

The sensitivity of each of these statistical comparisons in the form of a power calculation is provided by the last column of Table 5, which gives the percent differences between the averages from the two composites that would have been detected with a power of $90 \%$ (i.e., with a $90 \%$ probability) based upon the variation seen and the number of measurements remaining in the screened data.

Table 7. Average Screened Cobalt and Cesium Activity (in dpm per gram of slurry) by Type Composite

\begin{tabular}{|c|c|c|c|c|c|c|}
\hline Sample Type & 32 & 60 & & Percent (\%) & $\begin{array}{c}\text { Statistically } \\
\text { Significant } \\
\text { At the }\end{array}$ & $\begin{array}{c}\text { \% Difference } \\
\text { Detectable } \\
\text { With }\end{array}$ \\
\hline N Rows & 10 & 10 & Difference & Difference & $5 \%$ Level & $90 \%$ Power \\
\hline${ }^{60} \mathrm{Co}$ & $2.76 \mathrm{E}+06$ & $2.71 \mathrm{E}+06$ & $4.76 \mathrm{E}+04$ & $1.76 \%$ & No & $5.39 \%$ \\
\hline${ }^{137} \mathrm{Cs}$ & $1.93 \mathrm{E}+08$ & $1.87 \mathrm{E}+08$ & $6.14 \mathrm{E}+06$ & $3.28 \%$ & Yes & $3.92 \%$ \\
\hline
\end{tabular}

Although statistically significant, the difference between the two Tank 40 composites for the Cs-137 radioactivity is deemed to be of no practical concern.

\section{Weight Percent Soluble Solids of the Supernate}

Plots of the weight percent soluble solids data in Table A5 by type of composite are provided in Exhibit A14 of the Appendix. There is an indication of two outliers for the 30-hr values. The JMP results provided as part of Exhibit A14 show a statistically significant difference (at the 5\% significance level) of $1.2 \%$ between the means of the two types of composites. The 32 -hr composite average wt $\%$ soluble solids value (11.58 wt $\%$ ) is $1.2 \%$ above the $60-\mathrm{hr}$ average (11.44 wt\%).

Exhibit A15 in the Appendix provides comparisons between the two Tank 40 composites with the 2 questionable values for the 32-hr results removed. The results presented in this exhibit also indicate a statistically significant difference (at the 5\% significance level) between the means of the two types of composites. For these data, the difference is only $0.7 \%$. Although statistically significant, these differences $(1.2 \%$ for all of the data and $0.7 \%$ for the screened data) are seen as being of little practical concern.

\section{Elemental Analyses of Supernate from ICP-ES}

$\mathrm{Al}$ and $\mathrm{Na}$ elemental analyses were conducted on supernate samples from each of the composite using an aqua regia dissolution method and ICP-ES. As discussed above, these results are provided in Table A6 in the Appendix. 
Plots of these elemental concentrations (as grams/gram of slurry) by type of composite and ICP calibration are provided in Exhibit A16 in the Appendix. These plots indicate the potential for a pair of outliers for Al and $\mathrm{Na}$ in the 32-hour composite samples for the first ICP block. Initial statistical analyses were conducted on these results using all of the data. To make sure that the potential outliers are not driving the conclusions, an analysis with these values removed was also conducted.

Exhibit A17 in the Appendix provides the details of a statistical analysis of variance using the full set of Al and $\mathrm{Na}$ measurements. This analysis investigates for both composite and calibration effects. Table 8 summarizes the results from these analyses. As seen in Table 8, there are no statistically significant (at the 5\% level) differences between the means of the two composites for these elements measured using ICP-ES. The differences expressed as percentages of the $60-\mathrm{hr}$ averages are $-6.32 \%$ for $\mathrm{Al}$ and $-7.71 \%$ for $\mathrm{Na}$. The sensitivity of each of these statistical comparisons is explored in Exhibit A18 in the Appendix. The information in this exhibit is complemented by the last column of Table 8, which provides the percent difference between the averages from the two composites that would have been detected with a power of $90 \%$ (i.e., with a $90 \%$ probability) based upon the variation seen in the data and the number measurements.

Table 8. Average Al and Na Concentrations (as grams/gram of supernate) in the Supernate by Type of Composite

\begin{tabular}{|c|c|c|c|c|c|c|}
\hline Sample Type & 32 & 60 & & Percent (\%) & $\begin{array}{c}\text { Statistically } \\
\text { Significant } \\
\text { At the }\end{array}$ & $\begin{array}{c}\text { \% Difference } \\
\text { Detectable } \\
\text { With }\end{array}$ \\
\hline N Rows & 10 & 10 & Difference & Difference & $5 \%$ Level & 90\% Power \\
\hline Al & $2.05 \mathrm{E}-03$ & $2.18 \mathrm{E}-03$ & $-1.40 \mathrm{E}-04$ & $-6.32 \%$ & No & $16.4 \%$ \\
\hline $\mathbf{N a}$ & $3.91 \mathrm{E}-02$ & 4.24E-02 & $-3.27 \mathrm{E}-03$ & $-7.71 \%$ & No & $17.0 \%$ \\
\hline
\end{tabular}

Exhibit A19 in the Appendix provides the details of a statistical analysis of variance for the set of $\mathrm{Al}$ and $\mathrm{Na}$ measurements with the potential outliers removed. This analysis investigates for both composite and calibration effects. Table 9 summarizes the results from these analyses. As seen in Table 9, there are no statistically significant (at the 5\% level) differences between the means of the two composites for the elements measured using ICP-ES. The differences expressed as percentages of the 60-hr averages are both less than $1 \%$. A measure of the sensitivity of each of these statistical comparisons is provided by the last column of Table 9, which gives the percent difference between the averages from the two composites that would have been detected with a power of 90\% (i.e., with a $90 \%$ probability) based upon the variation seen and the number of measurements remaining in the screened data.

Table 9. Average Screened Al and Na Concentrations (as wt\% dried solids) in the Supernate by Type Composite

\begin{tabular}{|c|c|c|c|c|c|c|}
\hline Sample Type & 32 & 60 & & Percent $(\%)$ & $\begin{array}{c}\text { Statistically } \\
\text { Significant } \\
\text { At the }\end{array}$ & $\begin{array}{c}\text { \% Difference } \\
\text { Detectable } \\
\text { With }\end{array}$ \\
\hline N Rows & 10 & 10 & Difference & Difference & $5 \%$ Level & 90\% Power \\
\hline Al & $2.18 \mathrm{E}-03$ & $2.18 \mathrm{E}-03$ & $-4.00 \mathrm{E}-06$ & $-0.17 \%$ & No & $2.92 \%$ \\
\hline $\mathbf{N a}$ & 4.21E-02 & 4.24E-02 & $-2.51 \mathrm{E}-04$ & $-0.59 \%$ & No & $2.18 \%$ \\
\hline
\end{tabular}


WSRC-TR-2001-00565

Revision 0

Page 11 of 83

November 26, 2001

\section{Summary of Results and Conclusions}

A summary of the results from the statistical comparisons discussed in this technical report are presented in Table 10. The results from Table 10 may be summarized as follows:

- Using all of the measurement data,

- The difference between the weight percent (wt $\%$ ) slurry solids for the two Tank 40 samples was statistically significant at the 5\% level (i.e., with $95 \%$ confidence). However, the difference was only $1.23 \%$, which is judged to be small enough to be of no practical concern.

- The difference between the weight percent (wt $\%$ ) soluble solids in the supernate for the two Tank 40 samples was statistically significant at the 5\% level (i.e., with 95\% confidence). However, the difference was only $1.21 \%$, which is judged to be small enough to be of no practical concern. 


\section{Page 12 of 83}

\section{November 26, 2001}

Table 10. Summary of Comparisons Between of the Two Composites

\begin{tabular}{|c|c|c|c|c|c|c|c|}
\hline & $\begin{array}{c}\text { Unit of } \\
\text { Measure }\end{array}$ & \multicolumn{2}{|c|}{ Type of Composite } & Difference & Percent (\%) & $\begin{array}{c}\text { Statistically } \\
\text { Significant } \\
\text { At the } \\
\text { 5\% Level }\end{array}$ & $\begin{array}{l}\text { \% Difference } \\
\text { Detectable } \\
\text { With } \\
\text { 90\% Power }\end{array}$ \\
\hline Wt\% Slurry Solids & $\mathrm{wt} \%$ & $2.64 \mathrm{E}+01$ & $2.67 \mathrm{E}+01$ & $-3.27 \mathrm{E}-01$ & $-1.23 \%$ & Yes & $0.38 \%$ \\
\hline \multicolumn{8}{|l|}{ Elemental } \\
\hline Ag & wet wt $\%$ in slurry & $7.84 \mathrm{E}-03$ & $7.85 \mathrm{E}-03$ & $-1.00 \mathrm{E}-05$ & $-0.13 \%$ & No & $7.37 \%$ \\
\hline Al & wet wt $\%$ in slurry & $1.39 \mathrm{E}+00$ & $1.39 \mathrm{E}+00$ & $1.20 \mathrm{E}-04$ & $0.01 \%$ & No & $6.90 \%$ \\
\hline $\mathbf{C a}$ & wet wt $\%$ in slurry & $4.98 \mathrm{E}-01$ & $5.01 \mathrm{E}-01$ & $-2.36 \mathrm{E}-03$ & $-0.47 \%$ & No & $4.92 \%$ \\
\hline $\mathbf{F e}$ & wet wt $\%$ in slurry & $5.14 \mathrm{E}+00$ & $5.13 \mathrm{E}+00$ & $9.37 \mathrm{E}-03$ & $0.18 \%$ & No & $7.37 \%$ \\
\hline Mg & wet wt $\%$ in slurry & 4.14E-01 & 4.13E-01 & $1.14 \mathrm{E}-03$ & $0.28 \%$ & No & $7.46 \%$ \\
\hline Mn & wet wt $\%$ in slurry & 7.02E-01 & $6.99 \mathrm{E}-01$ & $2.80 \mathrm{E}-03$ & $0.40 \%$ & No & $7.52 \%$ \\
\hline $\mathbf{N a}$ & wet wt $\%$ in slurry & $4.20 \mathrm{E}+00$ & $4.18 \mathrm{E}+00$ & $2.43 \mathrm{E}-02$ & $0.58 \%$ & No & $6.38 \%$ \\
\hline $\mathbf{U}$ & wet wt $\%$ in slurry & $1.58 \mathrm{E}+00$ & $1.59 \mathrm{E}+00$ & $-1.12 \mathrm{E}-02$ & $-0.70 \%$ & No & $3.69 \%$ \\
\hline $\mathrm{Fe} / \mathrm{Na}$ & wet wt $\%$ in slurry & $1.22 \mathrm{E}+00$ & $1.23 \mathrm{E}+00$ & $-4.69 \mathrm{E}-03$ & $-0.38 \%$ & No & $2.26 \%$ \\
\hline \multicolumn{8}{|l|}{ Elemental (scrnd) } \\
\hline $\mathbf{A g}$ & wet wt $\%$ in slurry & $7.72 \mathrm{E}-03$ & 7.79E-03 & $-7.00 \mathrm{E}-05$ & $-0.86 \%$ & No & $5.02 \%$ \\
\hline Al & wet wt $\%$ in slurry & $1.35 \mathrm{E}+00$ & $1.37 \mathrm{E}+00$ & $-1.90 \mathrm{E}-02$ & $-1.38 \%$ & Yes & $1.55 \%$ \\
\hline $\mathbf{C a}$ & wet wt $\%$ in slurry & 4.90E-01 & $4.98 \mathrm{E}-01$ & $-7.26 \mathrm{E}-03$ & $-1.46 \%$ & Yes & $1.26 \%$ \\
\hline $\mathbf{F e}$ & wet wt $\%$ in slurry & $5.05 \mathrm{E}+00$ & $5.10 \mathrm{E}+00$ & $-4.52 \mathrm{E}-02$ & $-0.89 \%$ & No & $3.83 \%$ \\
\hline Mg & wet wt $\%$ in slurry & 4.03E-01 & $4.10 \mathrm{E}-01$ & $-6.80 \mathrm{E}-03$ & $-1.66 \%$ & No & $3.25 \%$ \\
\hline Mn & wet wt $\%$ in slurry & $6.84 \mathrm{E}-01$ & $6.95 \mathrm{E}-01$ & $-1.15 \mathrm{E}-02$ & $-1.66 \%$ & No & $3.28 \%$ \\
\hline $\mathrm{Na}$ & wet wt $\%$ in slurry & $4.11 \mathrm{E}+00$ & $4.15 \mathrm{E}+00$ & $-4.12 \mathrm{E}-02$ & $-0.99 \%$ & Yes & $1.23 \%$ \\
\hline $\mathbf{U}$ & wet wt $\%$ in slurry & $1.57 \mathrm{E}+00$ & $1.58 \mathrm{E}+00$ & $-7.00 \mathrm{E}-03$ & $-0.44 \%$ & No & $3.31 \%$ \\
\hline $\mathrm{Fe} / \mathrm{Na}$ & wet wt $\%$ in slurry & $1.22 \mathrm{E}+00$ & $1.23 \mathrm{E}+00$ & $-8.59 \mathrm{E}-03$ & $-0.70 \%$ & No & $2.10 \%$ \\
\hline \multicolumn{8}{|c|}{ Selected Fission Products \& Actinides } \\
\hline Tc-99 & 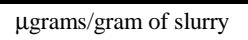 & $1.75 \mathrm{E}+00$ & $1.69 \mathrm{E}+00$ & $5.10 \mathrm{E}-02$ & $3.01 \%$ & No & $14.13 \%$ \\
\hline Ru-101 & $\mu$ grams/gram of slurry & $2.66 \mathrm{E}+01$ & $2.60 \mathrm{E}+01$ & $6.14 \mathrm{E}-01$ & $2.36 \%$ & No & $4.66 \%$ \\
\hline Ru-102 & $\mu$ grams/gram of slurry & $2.52 \mathrm{E}+01$ & $2.48 \mathrm{E}+01$ & $3.30 \mathrm{E}-01$ & $1.33 \%$ & No & $5.31 \%$ \\
\hline Rh-103 & $\mu$ grams/gram of slurry & $1.68 \mathrm{E}+01$ & $1.65 \mathrm{E}+01$ & $2.70 \mathrm{E}-01$ & $1.63 \%$ & No & $4.84 \%$ \\
\hline Ru-104 & $\mu$ grams/gram of slurry & $1.69 \mathrm{E}+01$ & $1.64 \mathrm{E}+01$ & $4.80 \mathrm{E}-01$ & $2.93 \%$ & No & $4.98 \%$ \\
\hline Pd-105 & $\mu$ grams/gram of slurry & $1.22 \mathrm{E}+00$ & $1.30 \mathrm{E}+00$ & $-8.10 \mathrm{E}-02$ & $-6.23 \%$ & No & $13.00 \%$ \\
\hline Ag-107 & $\mu$ grams/gram of slurry & $1.02 \mathrm{E}+01$ & $9.84 \mathrm{E}+00$ & $3.19 \mathrm{E}-01$ & $3.25 \%$ & No & $9.16 \%$ \\
\hline Ag-109 & $\mu$ grams/gram of slurry & $9.83 \mathrm{E}+00$ & $9.48 \mathrm{E}+00$ & $3.56 \mathrm{E}-01$ & $3.76 \%$ & No & $10.73 \%$ \\
\hline U-235 & $\mu$ grams/gram of slurry & $5.53 \mathrm{E}+01$ & $5.46 \mathrm{E}+01$ & 7.11E-01 & $1.30 \%$ & No & $4.77 \%$ \\
\hline U-238 & $\mu$ grams/gram of slurry & $1.35 \mathrm{E}+04$ & $1.34 \mathrm{E}+04$ & $4.01 \mathrm{E}+01$ & $0.30 \%$ & No & $6.71 \%$ \\
\hline Pu-239 & $\mu$ grams/gram of slurry & $2.18 \mathrm{E}+01$ & $2.09 \mathrm{E}+01$ & $9.19 \mathrm{E}-01$ & $4.40 \%$ & No & $7.49 \%$ \\
\hline \multicolumn{8}{|c|}{ Selected Fission Products \& Actinides (scrnd) } \\
\hline Tc-99 & $\mu$ grams/gram of slurry & $1.79 \mathrm{E}+00$ & $1.69 \mathrm{E}+00$ & $1.01 \mathrm{E}-01$ & $5.94 \%$ & No & $10.52 \%$ \\
\hline Ru-101 & $\mu$ grams/gram of slurry & $2.66 \mathrm{E}+01$ & $2.63 \mathrm{E}+01$ & $3.21 \mathrm{E}-01$ & $1.22 \%$ & No & $3.64 \%$ \\
\hline Ru-102 & $\mu$ grams/gram of slurry & $2.52 \mathrm{E}+01$ & $2.51 \mathrm{E}+01$ & $4.60 \mathrm{E}-02$ & $0.18 \%$ & No & $3.74 \%$ \\
\hline Rh-103 & $\mu$ grams/gram of slurry & $1.68 \mathrm{E}+01$ & $1.67 \mathrm{E}+01$ & 8.90E-02 & $0.53 \%$ & No & $3.19 \%$ \\
\hline Ru-104 & $\mu$ grams/gram of slurry & $1.69 \mathrm{E}+01$ & $1.65 \mathrm{E}+01$ & $3.24 \mathrm{E}-01$ & $1.96 \%$ & No & $3.94 \%$ \\
\hline Pd-105 & $\mu$ grams/gram of slurry & $1.22 \mathrm{E}+00$ & $1.28 \mathrm{E}+00$ & $-5.50 \mathrm{E}-02$ & $-4.35 \%$ & No & $14.14 \%$ \\
\hline Ag-107 & $\mu$ grams/gram of slurry & $1.00 \mathrm{E}+01$ & $9.84 \mathrm{E}+00$ & $2.09 \mathrm{E}-01$ & $2.12 \%$ & No & $8.82 \%$ \\
\hline Ag-109 & $\mu$ grams/gram of slurry & $9.71 \mathrm{E}+00$ & $9.48 \mathrm{E}+00$ & $2.30 \mathrm{E}-01$ & $2.43 \%$ & No & $10.30 \%$ \\
\hline U-235 & $\mu$ grams/gram of slurry & $5.53 \mathrm{E}+01$ & $5.52 \mathrm{E}+01$ & $7.70 \mathrm{E}-02$ & $0.14 \%$ & No & $2.72 \%$ \\
\hline U-238 & $\mu$ grams/gram of slurry & $1.32 \mathrm{E}+04$ & $1.34 \mathrm{E}+04$ & $-2.21 \mathrm{E}+02$ & $-1.65 \%$ & No & $5.52 \%$ \\
\hline Pu-239 & $\mu$ grams/gram of slurry & $2.18 \mathrm{E}+01$ & $2.11 \mathrm{E}+01$ & 7.19E-01 & $3.41 \%$ & No & $7.08 \%$ \\
\hline Cobalt-60 & dpm/gram of slurry & $2.70 \mathrm{E}+06$ & $2.62 \mathrm{E}+06$ & $8.10 \mathrm{E}+04$ & $3.09 \%$ & No & $9.26 \%$ \\
\hline Cesium-137 & dpm/gram of slurry & $1.87 \mathrm{E}+08$ & $1.83 \mathrm{E}+08$ & $4.50 \mathrm{E}+06$ & $2.46 \%$ & No & $7.23 \%$ \\
\hline Cobalt-60 (scrnd) & dpm/gram of slurry & $2.76 \mathrm{E}+06$ & $2.71 \mathrm{E}+06$ & $4.76 \mathrm{E}+04$ & $1.76 \%$ & No & $5.39 \%$ \\
\hline Cesium-137 (scrnd) & dpm/gram of slurry & $1.93 \mathrm{E}+08$ & $1.87 \mathrm{E}+08$ & $6.14 \mathrm{E}+06$ & $3.28 \%$ & Yes & $3.92 \%$ \\
\hline \multicolumn{8}{|l|}{ Supernate } \\
\hline Soluble Solids & $\mathrm{wt} \%$ & $1.16 \mathrm{E}+01$ & $1.14 \mathrm{E}+01$ & $1.38 \mathrm{E}-01$ & $1.21 \%$ & Yes & $1.48 \%$ \\
\hline Sol. Solids (scrnd) & $\mathrm{wt} \%$ & $1.15 \mathrm{E}+01$ & $1.14 \mathrm{E}+01$ & $8.60 \mathrm{E}-02$ & $0.75 \%$ & Yes & $0.97 \%$ \\
\hline Al & gram/gram of supernate & $2.05 \mathrm{E}-03$ & $2.18 \mathrm{E}-03$ & $-1.40 \mathrm{E}-04$ & $-6.32 \%$ & No & $16.41 \%$ \\
\hline $\mathbf{N a}$ & gram/gram of supernate & $3.91 \mathrm{E}-02$ & 4.24E-02 & $-3.27 \mathrm{E}-03$ & $-7.71 \%$ & No & $16.96 \%$ \\
\hline Al (scrnd) & gram/gram of supernate & $2.18 \mathrm{E}-03$ & $2.18 \mathrm{E}-03$ & $-4.00 \mathrm{E}-06$ & $-0.17 \%$ & No & $2.92 \%$ \\
\hline $\mathrm{Na}$ (scrnd) & gram/gram of supernate & $4.21 \mathrm{E}-02$ & $4.24 \mathrm{E}-02$ & $-2.51 \mathrm{E}-04$ & $-0.59 \%$ & No & $2.18 \%$ \\
\hline
\end{tabular}


- No other differences between the Tank 40 samples for any analyte considered as part of this study were statistically significant at the $5 \%$ level (i.e. with $95 \%$ confidence).

- The sensitivity of the process used to make the comparisons between the two Tank 40 samples (as revealed by Column 8 of Table 1) indicates that:

- For the analyses of each of the elemental concentrations in the slurry, any difference between the two samples of $7.52 \%$ or greater for one of these analytes would have been detected with at least a $90 \%$ probability.

- For the analyses of each of the other analytes (i.e., the selected fission products and actinides, Co-60, Cs-137, and the supernate measurements), any difference between the two samples of $16.96 \%$ or greater for one of these analytes would have been detected with at least a $90 \%$ probability. Thus, the sensitivity is not as good for some of these analytes as compared to the sensitivity for comparisons involving the elemental concentrations. This possibility was indicated in the TT\&QA plan [3].

- Using the data after the potential outlying measurements were removed,

- No potential outliers were seen in weight percent (wt\%) slurry solids for the two Tank 40 samples, so the screened results are the same as those presented above.

- The difference between the screened weight percent (wt\%) soluble solids in the supernate for the two Tank 40 samples was statistically significant at the 5\% level (i.e., with 95\% confidence). However, the difference was only $0.75 \%$, which is judged to be small enough to be of no practical concern.

- The difference between the screened elemental concentrations in the slurry for the two Tank 40 samples was statistically significant at the 5\% level (i.e., with 95\% confidence) for $\mathrm{Al}, \mathrm{Ca}$, and $\mathrm{Na}$. However, the differences were only $1.55 \%$ (for $\mathrm{Al}$ ), $1.26 \%$ (for $\mathrm{Ca}$ ), and $1.23 \%$ (for $\mathrm{Na}$ ), which are judged to be small enough to be of no practical concern.

- The sensitivity of the process used to make the comparisons between the two Tank 40 samples (as revealed by Column 8 of Table 1) indicates that:

- For the screened analyses of each of the elemental concentrations in the slurry, any difference between the two samples of $5.02 \%$ or greater for one of these analytes would have been detected with at least a $90 \%$ probability.

- For the screened analyses of each of the other analytes (i.e., the selected fission products and actinides, Co-60, Cs-137, and the supernate measurements), any difference between the two samples of $14.14 \%$ or greater for one of these analytes would have been detected with at least a $90 \%$ probability. The sensitivity of the comparisons between the two Tank 40 samples for these analytes improved for most of the analytes as a result of the screening process.

Based upon the results from the comparisons of the 32-hour and 60-hour Tank 40 samples summarized in this memorandum, these two samples of Tank 40 are judged to be essentially the same for the analytes considered as part of this task. 
WSRC-TR-2001-00565

Revision 0

Page 14 of 83

November 26, 2001

\section{ACKNOWLEDGEMENTS}

The authors wish to express their appreciation to those folks who worked long hours to see that this program was completed successfully and in a timely manner. These include S. J. Johnson and M. C. Lee of SCO, B. H. Burch, J. C. Hart, W. T. Boyce and C. C. DiPrete of ADS and A. L. Williams of WPT.

\section{REFERENCES}

\footnotetext{
${ }^{1}$ R. F. Swingle, "Task Technical and Quality Assurance Plan for Characterization and Washing of the Sludge Batch 2 Acceptance Sample,”WSRC-RP-2001-00176, Revision 0, May 30, 2001.

${ }^{2}$ J. E. Occhipinti, "Determine If A Statistical Difference in Composition Exists Between Samples Taken from Tank 40," HLW/DWPF/TTR-01-0025, Revision 0, July 11, 2001.
}

${ }^{3}$ SAS Institute, Inc. JMP® Statistics and Graphics Guide: JMP Version 4, SAS Institute, Inc., Cary, NC, 2000. 
WSRC-TR-2001-00565

Page 15 of 83

November 26, 2001

\section{APPROVALS}

\section{Authors}

R. F. Swingle, Waste Processing Technology

Date

N. E. Bibler, Immobilization Technology

Date

C. J. Coleman, Analytical Development

Date

T. L. Fellinger, Immobilization Technology

Date

T. B. Edwards, Statistical Consulting

Date

S. P. Harris, Statistical Consulting

Design Review per Manual E7, Procedure 2.40

\section{Management}

S. L. Marra, Level 4 Manager

Date

Immobilization Technology Section

R. C. Tuckfield, Level 3 Manager

Date

Statistical Consulting

W. B. Van Pelt, Level 4 Manager

Date

Waste Processing Technology Section 
WSRC-TR-2001-00565

Revision 0

Page 16 of 83

November 26, 2001

\section{Appendix}

Table A1. Weight Percent (wt\%) Solids of Slurry by Sample

\begin{tabular}{|cc|}
\hline Composite & Wt\% Solids \\
$30 \mathrm{hr}$ & 26.304 \\
$30 \mathrm{hr}$ & 26.461 \\
$30 \mathrm{hr}$ & 26.275 \\
$30 \mathrm{hr}$ & 26.368 \\
$30 \mathrm{hr}$ & 26.377 \\
$30 \mathrm{hr}$ & 26.370 \\
$30 \mathrm{hr}$ & 26.321 \\
$30 \mathrm{hr}$ & 26.222 \\
$30 \mathrm{hr}$ & 26.400 \\
$30 \mathrm{hr}$ & 26.412 \\
$62 \mathrm{hr}$ & 26.701 \\
$62 \mathrm{hr}$ & 26.674 \\
$62 \mathrm{hr}$ & 26.763 \\
$62 \mathrm{hr}$ & 26.792 \\
$62 \mathrm{hr}$ & 26.603 \\
$62 \mathrm{hr}$ & 26.622 \\
$62 \mathrm{hr}$ & 26.616 \\
$62 \mathrm{hr}$ & 26.675 \\
$62 \mathrm{hr}$ & 26.651 \\
$62 \mathrm{hr}$ & 26.685 \\
\hline
\end{tabular}




\section{Page 17 of 83}

November 26, 2001

Table A2. Elemental Concentrations as

Weight Percents (wt\%'s) of Slurry from ICP-ES Analyses

\begin{tabular}{|c|c|c|c|c|c|c|c|c|c|c|c|}
\hline $\begin{array}{c}\text { ADS } \\
\text { LIMS \# }\end{array}$ & $\begin{array}{l}\text { Type of } \\
\text { Sample }\end{array}$ & $\begin{array}{l}\text { Instrument } \\
\text { Calibration }\end{array}$ & Ag & Al & $\mathbf{C a}$ & $\mathbf{F e}$ & Mg & Mn & $\mathrm{Na}$ & $\mathbf{U}$ & $\mathrm{Fe} / \mathrm{Na}$ \\
\hline 300166374 & 60 & 1 & 0.0091 & 1.4220 & 0.5024 & 5.2896 & 0.4278 & 0.7198 & 4.1878 & 1.5280 & 1.2631 \\
\hline 300166375 & ARG-1/1 & 1 & 0.0148 & 2.5075 & 1.0695 & 10.7326 & 0.5720 & 1.6051 & 8.6477 & 0.2851 & 1.2411 \\
\hline 300166376 & 32 & 1 & 0.0090 & 1.4816 & 0.5084 & 5.5040 & 0.4425 & 0.7470 & 4.4110 & 1.5902 & 1.2478 \\
\hline 300166377 & 32 & 1 & 0.0084 & 1.3768 & 0.4909 & 5.0967 & 0.4103 & 0.6917 & 4.1197 & 1.4730 & 1.2372 \\
\hline 300166378 & 60 & 1 & 0.0087 & 1.3877 & 0.4976 & 5.1800 & 0.4163 & 0.6998 & 4.1183 & 1.4941 & 1.2578 \\
\hline 300166379 & 32 & 1 & 0.0082 & 1.3649 & 0.4872 & 5.0709 & 0.4060 & 0.6830 & 4.0923 & 1.4631 & 1.2391 \\
\hline 300166380 & 32 & 1 & 0.0086 & 1.3940 & 0.4946 & 5.1988 & 0.4153 & 0.6981 & 4.1629 & 1.4952 & 1.2488 \\
\hline 300166381 & 60 & 1 & 0.0084 & 1.3912 & 0.4983 & 5.1872 & 0.4124 & 0.6916 & 4.1460 & 1.4987 & 1.2511 \\
\hline 300166382 & Blk 1 & 1 & 0.0023 & 0.0116 & 0.0000 & 0.0104 & 0.0009 & 0.0007 & 0.0148 & 0.0398 & 0.7027 \\
\hline 300166383 & 60 & 1 & 0.0087 & 1.4172 & 0.5057 & 5.3161 & 0.4231 & 0.7079 & 4.2042 & 1.5262 & 1.2645 \\
\hline 300166384 & ARG-1/1 & 1 & 0.0138 & 2.4961 & 1.0631 & 10.6257 & 0.5562 & 1.5533 & 8.5400 & 0.2899 & 1.2446 \\
\hline 300166385 & 32 & 1 & 0.0090 & 1.3927 & 0.4956 & 5.3080 & 0.4214 & 0.7057 & 4.1260 & 1.5622 & 1.2865 \\
\hline 300166386 & 60 & 1 & 0.0088 & 1.5020 & 0.5150 & 5.6133 & 0.4437 & 0.7438 & 4.4536 & 1.6084 & 1.2604 \\
\hline 300166387 & 60 & 2 & 0.0075 & 1.3889 & 0.5123 & 5.1157 & 0.4186 & 0.7164 & 4.1900 & 1.6600 & 1.2209 \\
\hline 300166388 & 32 & 2 & 0.0069 & 1.3219 & 0.4866 & 4.7887 & 0.3911 & 0.6693 & 4.0967 & 1.6300 & 1.1689 \\
\hline 300166389 & 60 & 2 & 0.0067 & 1.3447 & 0.4949 & 4.8601 & 0.3947 & 0.6764 & 4.1281 & 1.6300 & 1.1773 \\
\hline 300166390 & 32 & 2 & 0.0080 & 1.5622 & 0.5534 & 5.7798 & 0.4689 & 0.8016 & 4.7422 & 1.6800 & 1.2188 \\
\hline 300166391 & Blk 2 & 2 & 0.0000 & 0.0076 & 0.0000 & 0.0218 & 0.0017 & 0.0025 & 0.0166 & 0.0000 & 1.3133 \\
\hline 300166392 & ARG-1/2 & 2 & 0.0000 & 2.3618 & 1.0266 & 10.1205 & 0.5398 & 1.5410 & 8.4957 & 0.0000 & 1.1913 \\
\hline 300166393 & 32 & 2 & 0.0068 & 1.3253 & 0.4931 & 4.9200 & 0.3981 & 0.6819 & 4.0776 & 1.6400 & 1.2066 \\
\hline 300166394 & 60 & 2 & 0.0069 & 1.3439 & 0.4971 & 5.0122 & 0.4048 & 0.6927 & 4.1002 & 1.6400 & 1.2224 \\
\hline 300166395 & 60 & 2 & 0.0069 & 1.3399 & 0.4905 & 4.8663 & 0.3924 & 0.6706 & 4.1007 & 1.6400 & 1.1867 \\
\hline 300166396 & ARG-1/2 & 2 & 0.0000 & 2.3668 & 0.9822 & 10.0126 & 0.5358 & 1.5135 & 8.5133 & 0.0000 & 1.1761 \\
\hline 300166397 & 32 & 2 & 0.0066 & 1.3175 & 0.4842 & 4.8117 & 0.3871 & 0.6606 & 4.0867 & 1.6100 & 1.1774 \\
\hline 300166398 & 60 & 2 & 0.0068 & 1.3399 & 0.4936 & 4.8820 & 0.3920 & 0.6696 & 4.1236 & 1.6500 & 1.1839 \\
\hline 300166399 & 32 & 2 & 0.0069 & 1.3417 & 0.4898 & 4.9376 & 0.3965 & 0.6777 & 4.0800 & 1.6200 & 1.2102 \\
\hline
\end{tabular}




\section{Page 18 of 83}

\section{November 26, 2001}

Appendix (continued)

Table A3. Noble Metal Concentrations (as micrograms per grams of slurry) from ICP-MS Analyses

\begin{tabular}{|c|c|c|c|c|c|c|c|c|c|c|c|c|c|}
\hline $\begin{array}{c}\text { ADS } \\
\text { LIMS \# }\end{array}$ & $\begin{array}{c}\text { Composite } \\
\text { Type }\end{array}$ & Calibration & Tc-99 & Ru-101 & Ru-102 & Rh-103 & Ru-104 & Pd-105 & Ag-107 & Ag-109 & U-235 & U-238 & Pu-239 \\
\hline 300166374 & 60 & 1 & 1.8031 & 27.088 & 25.512 & 17.324 & 16.531 & 1.4089 & 8.782 & 8.587 & 53.197 & 13571.04 & 19.565 \\
\hline 300166375 & ARG1-1 & 1 & 0.4099 & & & & 0.099 & 0.0893 & 0.511 & 0.165 & & 653.24 & 25.756 \\
\hline 300166376 & 32 & 1 & 2.0030 & 27.134 & 26.132 & 17.576 & 17.890 & 1.4292 & 10.149 & 9.676 & 55.619 & 14172.20 & 21.744 \\
\hline 300166377 & 32 & 1 & 1.2962 & 26.339 & 24.749 & 16.835 & 16.764 & 1.3040 & 9.476 & 9.938 & 51.540 & 12154.89 & 20.647 \\
\hline 300166378 & 60 & 1 & 1.5618 & 26.393 & 25.635 & 16.708 & 17.183 & 1.2813 & 10.349 & 10.482 & 53.356 & 13238.82 & 22.085 \\
\hline 300166379 & 32 & 1 & 1.7086 & 26.764 & 25.282 & 17.081 & 17.020 & 1.3689 & 10.018 & 10.156 & 53.260 & 12395.48 & 21.014 \\
\hline 300166380 & 32 & 1 & 1.6121 & 27.648 & 24.798 & 16.959 & 16.607 & 1.0457 & 10.052 & 10.430 & 53.674 & 13069.60 & 21.573 \\
\hline 300166381 & 60 & 1 & 1.7476 & 26.723 & 25.095 & 16.713 & 17.072 & 1.4224 & 9.411 & 9.227 & 53.775 & 12910.29 & 19.918 \\
\hline 300166382 & Blk1 & 1 & 0.2000 & . & & & 0.124 & & . & 0.095 & . & 6.36 & 0.155 \\
\hline 300166383 & 60 & 1 & 1.6567 & 27.251 & 26.411 & 17.378 & 16.975 & 1.3754 & 10.081 & 9.597 & 52.487 & 12572.77 & 19.839 \\
\hline 300166384 & ARG1-1 & 1 & 0.2528 & - & & & & 0.0959 & 0.762 & 0.228 & - & 8.42 & 0.734 \\
\hline 300166385 & 32 & 1 & 1.7827 & 27.860 & 26.163 & 17.526 & 17.466 & 1.1941 & 11.098 & 11.255 & 54.069 & 12721.09 & 21.861 \\
\hline 300166386 & 60 & 1 & 1.7512 & 26.959 & 26.152 & 17.632 & 17.367 & 1.4778 & 10.574 & 10.704 & 54.571 & 13309.23 & 21.695 \\
\hline 300166387 & 60 & 1 & 1.6526 & 26.137 & 24.907 & 16.612 & 16.391 & 1.5030 & 10.058 & 9.783 & 51.863 & 12484.92 & 21.023 \\
\hline 300166387 & 60 & 2 & 1.7861 & 25.461 & 24.324 & 16.585 & 15.494 & 1.1617 & 10.496 & 9.494 & 56.967 & 14027.13 & 21.303 \\
\hline 300166388 & 32 & 1 & 1.7863 & 28.426 & 26.602 & 18.108 & 18.151 & 1.4414 & 10.967 & 11.158 & 55.307 & 13725.64 & 22.190 \\
\hline 300166388 & 32 & 2 & 1.8361 & 25.976 & 23.913 & 16.132 & 16.378 & 1.0899 & 10.344 & 9.716 & 56.068 & 13842.74 & 20.340 \\
\hline 300166389 & 60 & 2 & 1.7424 & 25.319 & 24.787 & 15.753 & 16.218 & 1.3189 & 10.319 & 10.067 & 56.707 & 13717.40 & 21.699 \\
\hline 300166390 & 32 & 2 & 1.9142 & 26.640 & 25.473 & 16.744 & 17.157 & 1.2510 & 10.678 & 9.922 & 58.245 & 14029.69 & 21.527 \\
\hline 300166391 & Blk2 & 2 & 0.3111 & 0.200 & . & . & 0.181 & . & 0.137 & 0.131 & 0.137 & 28.71 & 0.408 \\
\hline 300166392 & ARG1-2 & 2 & 12.7608 & . & . & . & . & . & 30.441 & 9.839 & . & 12.89 & 0.802 \\
\hline 300166393 & 32 & 2 & 1.8967 & 25.957 & 25.416 & 16.395 & 16.702 & 1.1820 & 10.114 & 8.878 & 56.339 & 13924.99 & 23.552 \\
\hline 300166394 & 60 & 2 & 1.7236 & 26.477 & 24.299 & 16.308 & 15.988 & 1.3213 & 9.294 & 8.451 & 57.465 & 14569.72 & 21.803 \\
\hline 300166395 & 60 & 2 & 1.5737 & 22.832 & 21.837 & 14.515 & 14.591 & 1.1049 & 8.987 & 8.621 & 50.519 & 12907.48 & 19.567 \\
\hline 300166396 & ARG1-2 & 2 & 0.2615 & 0.131 & & & & & 1.012 & 0.197 & 0.173 & 30.16 & 0.852 \\
\hline 300166397 & 32 & 2 & 1.6830 & 26.168 & 24.651 & 16.549 & 16.264 & 1.2816 & 9.727 & 9.342 & 57.027 & 14878.79 & 23.512 \\
\hline 300166398 & 60 & 2 & 1.5928 & 25.649 & 24.346 & 16.556 & 16.407 & 1.1362 & 10.070 & 9.525 & 56.495 & 13573.04 & 21.546 \\
\hline 300166399 & 32 & 2 & 1.7162 & 25.801 & 25.119 & 16.375 & 16.375 & 1.0513 & 9.902 & 9.003 & 56.806 & 13608.32 & 22.445 \\
\hline
\end{tabular}


WSRC-TR-2001-00565

Revision 0

Page 19 of 83

November 26, 2001

Appendix (continued)

Table A4. Activity Measured by Gamma Scan

\begin{tabular}{|ccccc|}
\hline ADS LIMS \# & Composite Type & Calibration & Co-60 (dpm/g) & Cs-137 (dpm/g) \\
300166374 & 60 & 1 & $2.66 \mathrm{E}+06$ & $1.85 \mathrm{E}+08$ \\
300166375 & ARG1-1 & 1 &. & $1.77 \mathrm{E}+06$ \\
300166376 & 32 & 1 & $2.84 \mathrm{E}+06$ & $1.98 \mathrm{E}+08$ \\
300166377 & 32 & 1 & $2.68 \mathrm{E}+06$ & $1.91 \mathrm{E}+08$ \\
300166378 & 60 & 1 & $2.36 \mathrm{E}+06$ & $1.88 \mathrm{E}+08$ \\
300166379 & 32 & 1 & $2.83 \mathrm{E}+06$ & $1.92 \mathrm{E}+08$ \\
300166380 & 32 & 1 & $2.71 \mathrm{E}+06$ & $1.75 \mathrm{E}+08$ \\
300166381 & 60 & 1 & $2.47 \mathrm{E}+06$ & $1.89 \mathrm{E}+08$ \\
300166382 & Blk1 & 1 &. & $1.79 \mathrm{E}+03$ \\
300166383 & 60 & 1 & $2.72 \mathrm{E}+06$ & $1.92 \mathrm{E}+08$ \\
300166384 & ARG1-1 & 1 &. & $4.64 \mathrm{E}+05$ \\
300166385 & 32 & 1 & $2.70 \mathrm{E}+06$ & $1.94 \mathrm{E}+08$ \\
300166386 & 60 & 1 & $2.81 \mathrm{E}+06$ & $1.78 \mathrm{E}+08$ \\
300166387 & 60 & 1 & $2.67 \mathrm{E}+06$ & $1.72 \mathrm{E}+08$ \\
300166388 & 32 & 1 & $2.49 \mathrm{E}+06$ & $1.90 \mathrm{E}+08$ \\
300166389 & 60 & 2 & $2.60 \mathrm{E}+06$ & $1.85 \mathrm{E}+08$ \\
300166390 & 32 & 2 & $2.69 \mathrm{E}+06$ & $1.90 \mathrm{E}+08$ \\
300166391 & $\mathrm{Blk} 2$ & 2 & $\cdot$ & $1.17 \mathrm{E}+03$ \\
300166392 & ARG1-2 & 2 &. & $1.17 \mathrm{E}+06$ \\
300166393 & 32 & 2 & $2.76 \mathrm{E}+06$ & $1.99 \mathrm{E}+08$ \\
300166394 & 60 & 2 & $2.38 \mathrm{E}+06$ & $1.90 \mathrm{E}+08$ \\
300166395 & 60 & 2 & $2.70 \mathrm{E}+06$ & $1.81 \mathrm{E}+08$ \\
300166396 & ARG1-2 & 2 &. & $4.11 \mathrm{E}+06$ \\
300166397 & 32 & 2 & $2.44 \mathrm{E}+06$ & $1.76 \mathrm{E}+08$ \\
300166398 & 60 & 2 & $2.82 \mathrm{E}+06$ & $1.72 \mathrm{E}+08$ \\
300166399 & 32 & 2 & $2.86 \mathrm{E}+06$ & $1.72 \mathrm{E}+08$ \\
\hline
\end{tabular}


WSRC-TR-2001-00565

Revision 0

Page 20 of 83

November 26, 2001

\section{Appendix (continued)}

Table A5. Weight Percent (wt\%) Soluble Solids of Supernate by Composite Sample

\begin{tabular}{|cc|}
\hline Composite & Wt\% Solids \\
$32 \mathrm{hr}$ & 11.520 \\
$32 \mathrm{hr}$ & 11.536 \\
$32 \mathrm{hr}$ & 11.659 \\
$32 \mathrm{hr}$ & 11.547 \\
$32 \mathrm{hr}$ & 11.531 \\
$32 \mathrm{hr}$ & 11.552 \\
$32 \mathrm{hr}$ & 11.558 \\
$32 \mathrm{hr}$ & 11.480 \\
$32 \mathrm{hr}$ & 11.922 \\
$32 \mathrm{hr}$ & 11.497 \\
$60 \mathrm{hr}$ & 11.460 \\
$60 \mathrm{hr}$ & 11.391 \\
$60 \mathrm{hr}$ & 11.438 \\
$60 \mathrm{hr}$ & 11.383 \\
$60 \mathrm{hr}$ & 11.524 \\
$60 \mathrm{hr}$ & 11.498 \\
$60 \mathrm{hr}$ & 11.587 \\
$60 \mathrm{hr}$ & 11.302 \\
$60 \mathrm{hr}$ & 11.491 \\
$60 \mathrm{hr}$ & 11.345 \\
\hline
\end{tabular}

Table A6. Elemental Concentrations as Weight Percents (wt\%'s) of Slurry from ICP-ES Analyses

\begin{tabular}{|c|c|c|c|c|}
\hline $\begin{array}{c}\text { ADS } \\
\text { LIMS \# }\end{array}$ & $\begin{array}{l}\text { Type of } \\
\text { Sample }\end{array}$ & $\begin{array}{l}\text { Instrument } \\
\text { Calibration }\end{array}$ & Al & $\mathbf{N a}$ \\
\hline 300166348 & 60 & 1 & 0.00219 & 0.0424 \\
\hline 300166350 & 32 & 1 & 0.00217 & 0.0422 \\
\hline 300166351 & 32 & 1 & 0.00113 & 0.0221 \\
\hline 300166352 & 60 & 1 & 0.00218 & 0.0424 \\
\hline 300166353 & 32 & 1 & 0.00218 & 0.0425 \\
\hline 300166354 & 32 & 1 & 0.00186 & 0.0319 \\
\hline 300166355 & 60 & 1 & 0.00216 & 0.0423 \\
\hline 300166357 & 60 & 1 & 0.00217 & 0.0423 \\
\hline 300166359 & 32 & 1 & 0.00215 & 0.0419 \\
\hline 300166360 & 60 & 1 & 0.00215 & 0.0420 \\
\hline 300166361 & 60 & 2 & 0.00227 & 0.0437 \\
\hline 300166362 & 32 & 2 & 0.00221 & 0.0426 \\
\hline 300166363 & 60 & 2 & 0.00220 & 0.0421 \\
\hline 300166364 & 32 & 2 & 0.00212 & 0.0407 \\
\hline 300166367 & 32 & 2 & 0.00221 & 0.0423 \\
\hline 300166368 & 60 & 2 & 0.00221 & 0.0423 \\
\hline 300166369 & 60 & 2 & 0.00220 & 0.0423 \\
\hline 300166371 & 32 & 2 & 0.00221 & 0.0424 \\
\hline 300166372 & 60 & 2 & 0.00210 & 0.0421 \\
\hline 300166373 & 32 & 2 & 0.00221 & 0.0426 \\
\hline
\end{tabular}


WSRC-TR-2001-00565

Revision 0

Page 21 of 83

November 26, 2001

.Appendix (continued)

\section{Exhibit A1. Plots of the Weight Percent (wt\%) Solids Values by Type of Composite}

Oneway Analysis of wt\% solids By Composite

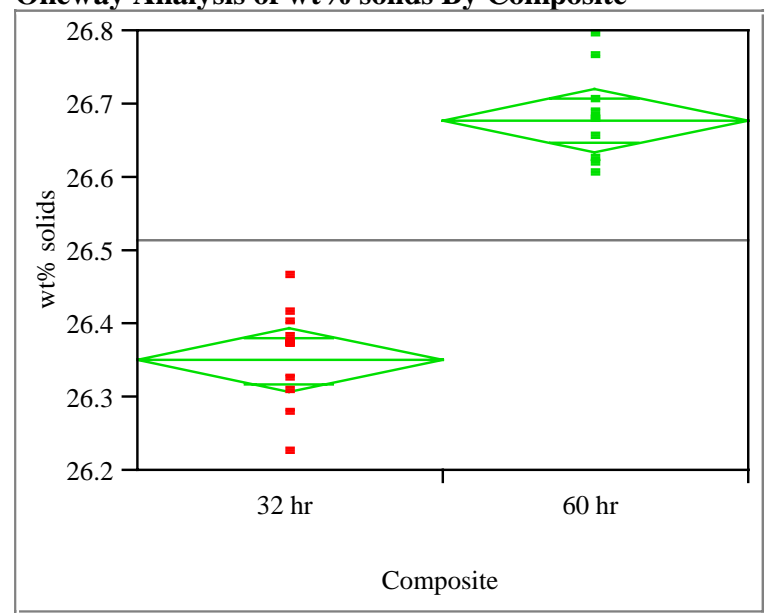

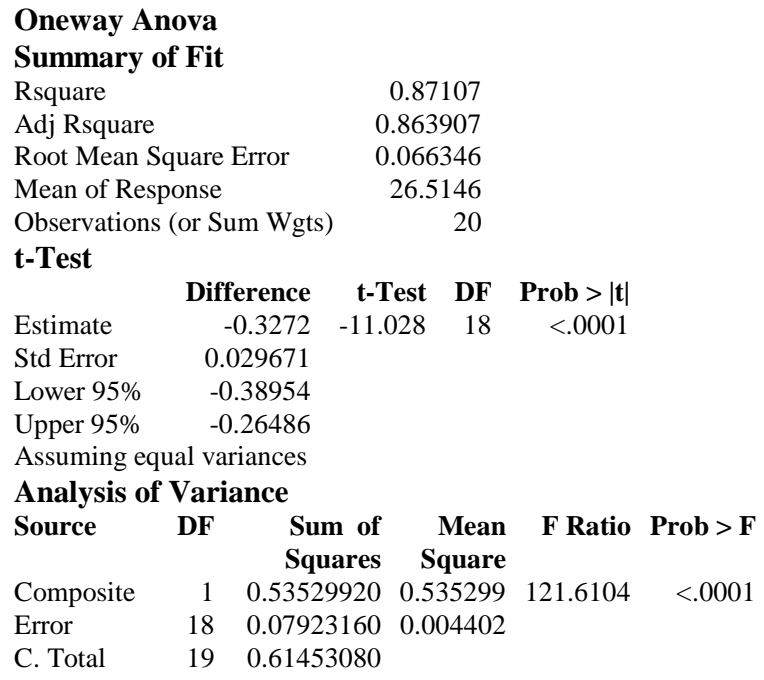

Means for Oneway Anova

$\begin{array}{lrrrrr}\text { Level } & \text { Number } & \text { Mean } & \text { Std Error } & \text { Lower 95\% } & \text { Upper 95\% } \\ 30 \mathrm{hr} & 10 & 26.3510 & 0.02098 & 26.307 & 26.395\end{array}$

$\begin{array}{llllll}62 \mathrm{hr} & 10 & 26.6782 & 0.02098 & 26.634 & 26.722\end{array}$

Std Error uses a pooled estimate of error variance

Tests that the Variances are Equal

Level Count Std Dev MeanAbsDif to Mean MeanAbsDif to Median

$\begin{array}{lllll}30 \mathrm{hr} & 10 & 0.0707688 & 0.0564000 & 0.0530000\end{array}$

$\begin{array}{lllll}62 \mathrm{hr} & 10 & 0.0616059 & 0.0456400 & 0.0450000\end{array}$

Test F Ratio DFNum DFDen Prob>F

$\begin{array}{lllll}\text { O’Brien[.5] } & 0.2159 & 1 & 18 & 0.6477\end{array}$

$\begin{array}{lllll}\text { Brown-Forsythe } & 0.1681 & 1 & 18 & 0.6867\end{array}$

$\begin{array}{lllll}\text { Levene } & 0.3918 & 1 & 18 & 0.5392\end{array}$

$\begin{array}{llll}\text { Bartlett } & 0.1634 & 1 & 0.6860\end{array}$

Welch Anova testing Means Equal, allowing Std Devs Not Equal

$\begin{array}{rrrr}\text { F Ratio } & \text { DFNum } & \text { DFDen } & \text { Prob }>\text { F } \\ 121.6104 & 1 & 17.665 & <.0001\end{array}$

t-Test

11.0277 
Page 22 of 83

November 26, 2001

Appendix (continued)

Exhibit A2. Plots of the Elemental Concentrations as Wet Weight Percents (wt\%'s) of Slurry by Type of Composite and ICP Calibration Block
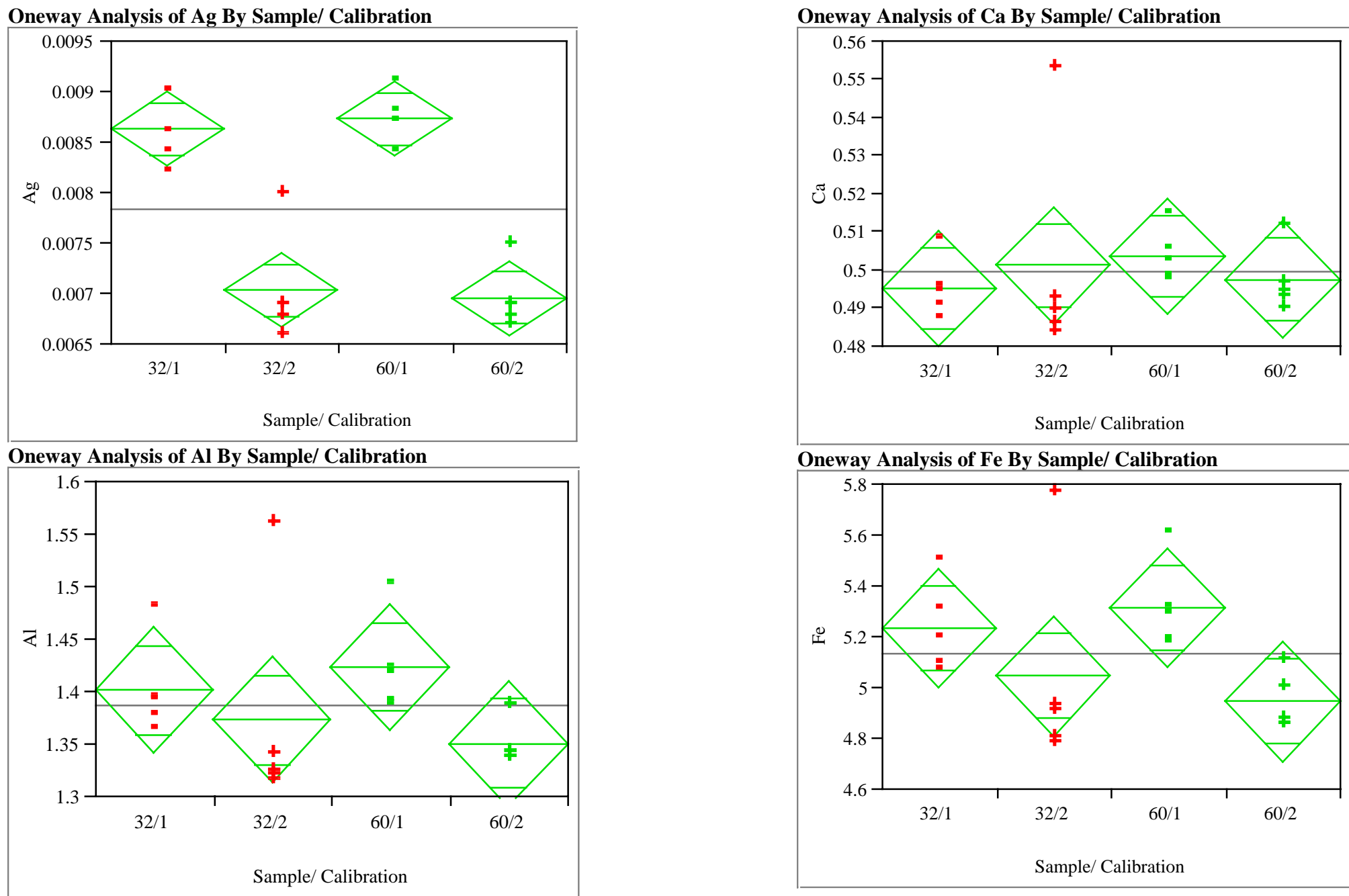
Page 23 of 83

November 26, 2001

Appendix (continued)

Exhibit A2. Plots of the Elemental Concentrations as Wet Weight Percents (wt\%'s) of Slurry by Type of Composite and ICP Calibration Block (continued)
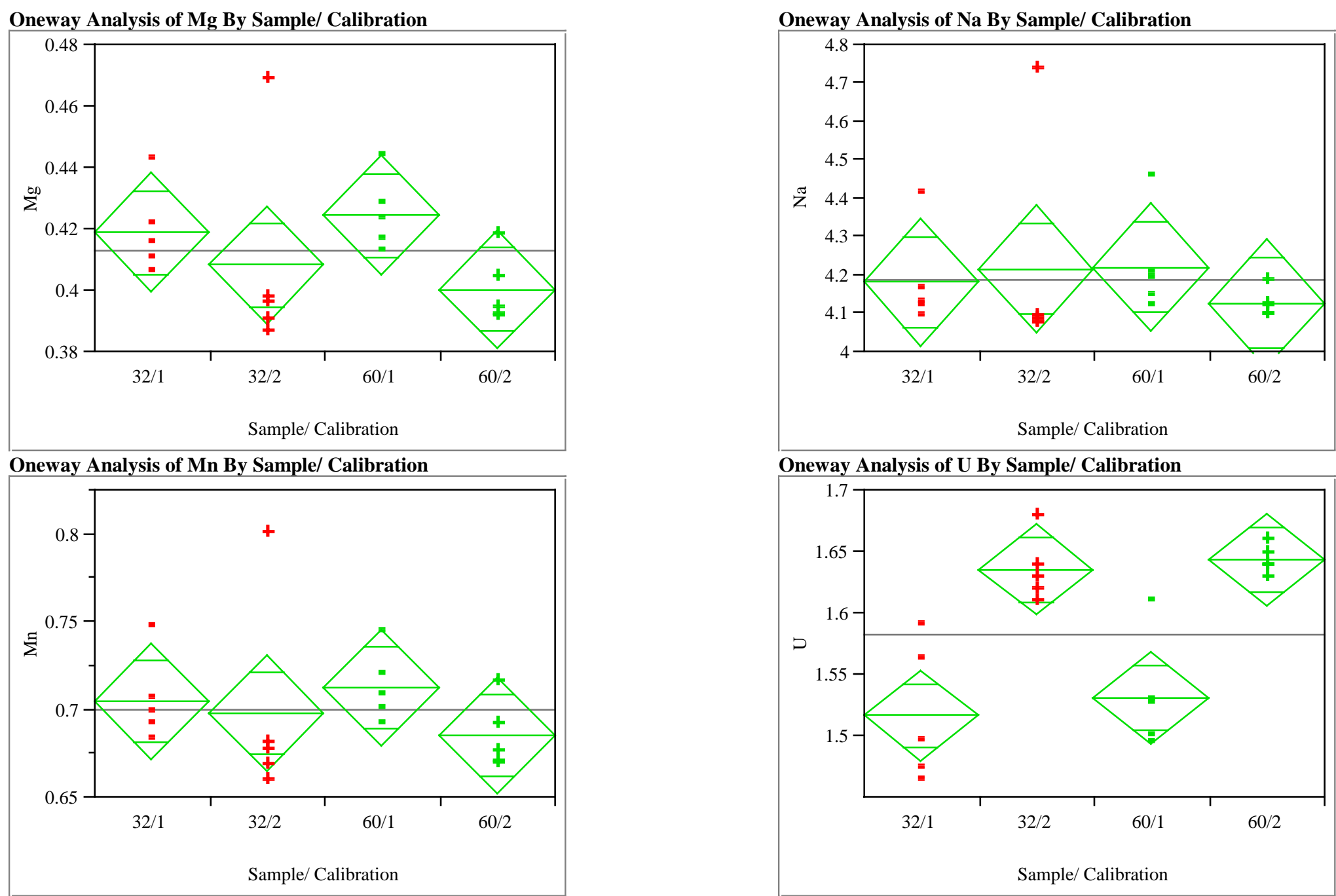
Page 24 of 83

November 26, 2001

\section{Appendix (continued)}

Exhibit A2. Plots of the Elemental Concentrations as Wet Weight Percents (wt\%'s) of Slurry by Type of Composite and ICP Calibration Block (continued)

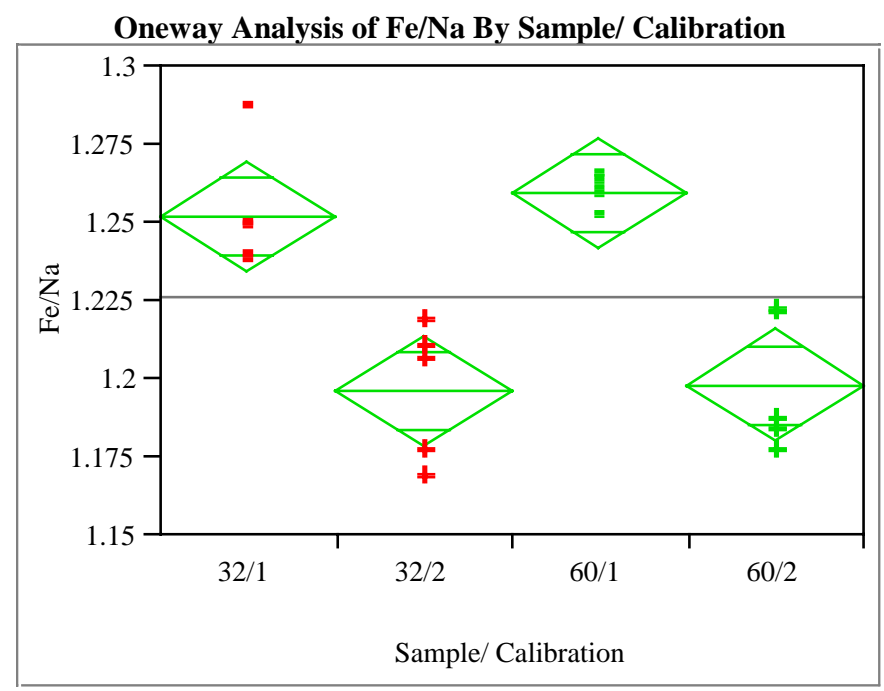


WSRC-TR-2001-00565

Revision 0

Page 25 of 83

November 26, 2001

\section{Appendix (continued)}

\section{Exhibit A3. Statistical Analyses of the Elemental Concentrations as Wet Weight Percents (wt\%'s) of Slurry by Type of Composite and ICP Calibration Block (continued)}

\section{Response Ag}

Whole Model

Actual by Predicted Plot

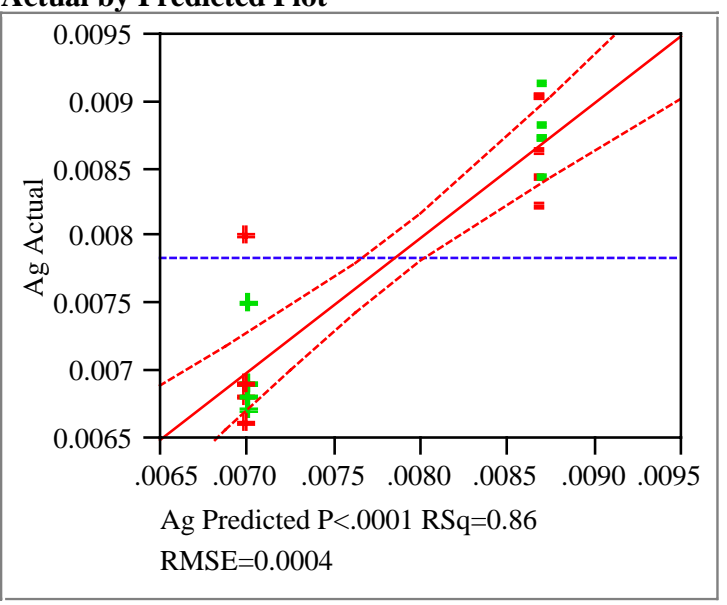

Residual by Predicted Plot

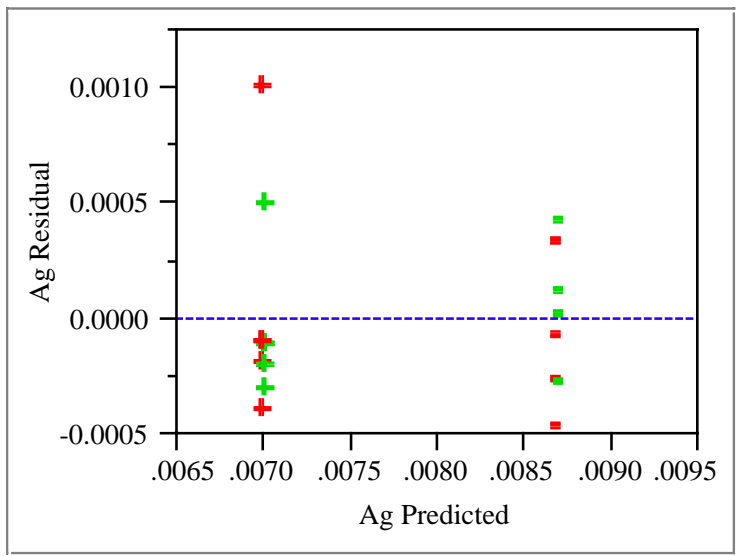

Summary of Fit

R-square is the portion of variation attributed to the model, between 0 and 1. Root Mean Squared Error "RMSE" estimates the standard deviation of the residual.

RSquare 0.855688

RSquare Adj 0.83871

Root Mean Square Error 0.000376

Mean of Response 0.007845

Observations (or Sum Wgts) 20

Analysis of Variance

The test that the whole model fits better than a simple mean, i.e. testing that all the parameters are zero except the intercept

Source DF Sum of Squares Mean Square F Ratio

$\begin{array}{lllll}\text { Model } & 2 & 0.00001428 & 0.0000071 & 50.4000\end{array}$

$\begin{array}{lllll}\text { Error } & 17 & 0.00000241 & 0.0000001 & \text { Prob }>\text { F }\end{array}$

$\begin{array}{llll}\text { C. Total } & 19 & 0.00001669 & <.0001\end{array}$

\section{Lack Of Fit}

Using replicated points as the part of residual error that does not depend on the form of the model so that you can test for the adequacy of the form of the model.

Source

Sum of Squares

Lack Of Fit 1

Pure Error 0.00000004 0.00000237

Total Error 0.00000241

$$
\begin{array}{rr}
\text { Mean Square } & \text { F Ratio } \\
4.05 \mathrm{e}-8 & 0.2736 \\
0.0000001 & \text { Prob > F } \\
& 0.6081 \\
& \text { Max RSq } \\
& 0.8581
\end{array}
$$

Parameter Estimates

Term

Intercept

Sample Type[32]

Calibration[1]

Effect Tests

Source

Sample Type

Calibration

$\begin{array}{rrrr}\text { Estimate } & \text { Std Error } & \text { t Ratio } & \text { Prob }>|\mathbf{t}| \\ 0.007845 & 0.000084 & 93.21 & <.0001 \\ -0.000005 & 0.000084 & -0.06 & 0.9533\end{array}$

$\begin{array}{rrrr}0.000845 & 0.000084 & 10.04 & <.0001\end{array}$

Nparm DF Sum of Squares

F Ratio Prob > F

$0.0035 \quad 0.9533$

$00.7966<.0001$ 
WSRC-TR-2001-00565

Revision 0

Page 26 of 83

November 26, 2001

\section{Appendix (continued)}

\section{Exhibit A3. Statistical Analyses of the Elemental Concentrations as Wet Weight Percents (wt\%'s) of Slurry by Type of Composite and ICP Calibration Block (continued)}

\section{Response Al}

Whole Model

Actual by Predicted Plot

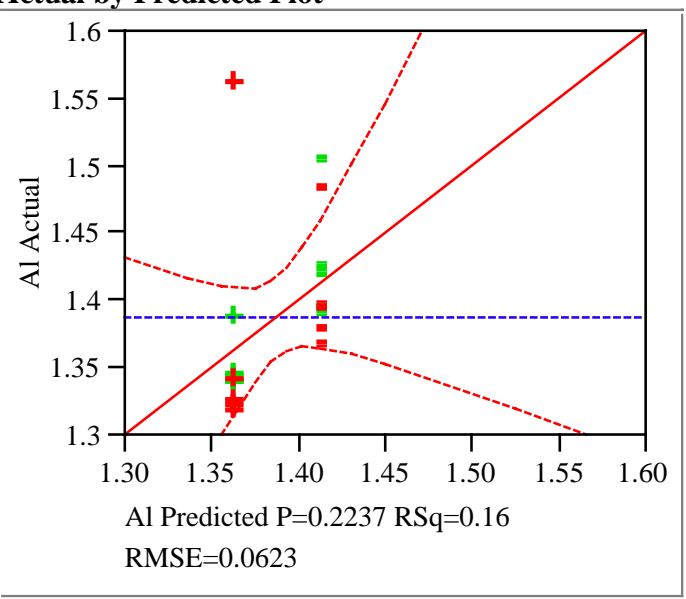

Residual by Predicted Plot

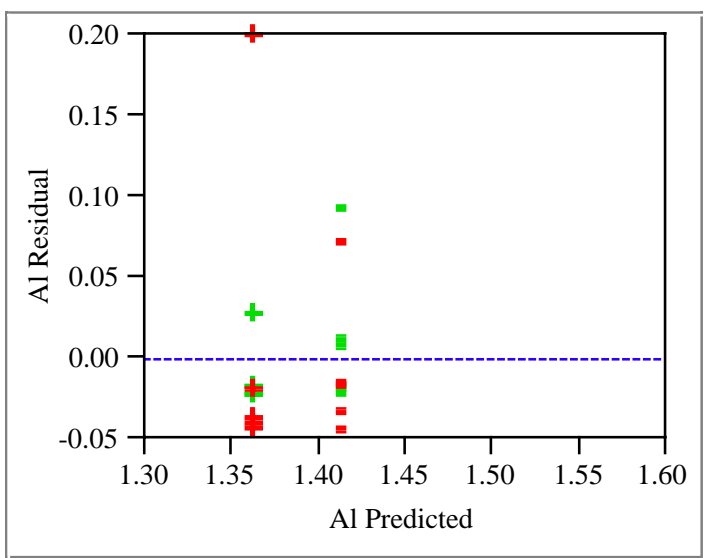

Summary of Fit

R-square is the portion of variation attributed to the model, between 0 and 1. Root Mean Squared Error "RMSE" estimates the standard deviation of the residual.
RSquare
0.161511
RSquare Adj
0.062866
0.062303
Root Mean Square Error
1.3878
Mean of Response
20
Analysis of Variance

The test that the whole model fits better than a simple mean, i.e. testing that all the parameters are zero except the intercept

Source DF Sum of Squares Mean Square F Ratio

$\begin{array}{lllll}\text { Model } & 2 & 0.01271095 & 0.006355 & 1.6373\end{array}$

$\begin{array}{lllll}\text { Error } & 17 & 0.06598913 & 0.003882 & \text { Prob > F }\end{array}$

$\begin{array}{llll}\text { C. Total } & 19 & 0.07870008 & 0.2237\end{array}$

\section{Lack Of Fit}

Using replicated points as the part of residual error that does not depend on the form of the model so that you can test for the adequacy of the form of the model.

Source

Lack Of Fit

Pure Error $\begin{array}{rr}\text { DF } & \text { Sum of Squares } \\ 1 & 0.00245090\end{array}$

Mean Square

F Ratio

0.002451

0.6172

Total Error

0.06353823

0.003971

Prob > F

0.4436

Max RSq

0.1927

Parameter Estimates

Term

Intercept

Sample Type[32]

Calibration[1]

Effect Tests

Source

Sample Type

Calibration
Estimate

$$
1.3878
$$

0.00006

0.02521

Nparm

DF Sum of Squares

Std Error t Ratio Prob $>|\mathbf{t}|$

$0.013931 \quad 99.62 \quad<.0001$

$\begin{array}{lll}0.013931 & 0.00 \quad 0.9966\end{array}$

$\begin{array}{lll}0.013931 & 1.81 & 0.0881\end{array}$

$\begin{array}{rrrrr}1 & 1 & 0.00000007 & 0.0000 & 0.9966 \\ 1 & 1 & 0.01271088 & 3.2746 & 0.0881\end{array}$


WSRC-TR-2001-00565

Revision 0

Page 27 of 83

November 26, 2001

\section{Appendix (continued)}

\section{Exhibit A3. Statistical Analyses of the Elemental Concentrations as Wet Weight Percents (wt\%'s) of Slurry by Type of Composite and ICP Calibration Block (continued)}

\section{Response Ca}

Whole Model

Actual by Predicted Plot



Residual by Predicted Plot



\section{Summary of Fit}

R-square is the portion of variation attributed to the model, between 0 and 1. Root Mean Squared Error "RMSE" estimates the standard deviation of the residual.
RSquare
0.006354
RSquare Adj
$-0.11055$
Root Mean Square Erro 0.016006
Mean of Response 0.49956
Observations (or Sum Wgts) 20

Analysis of Variance

The test that the whole model fits better than a simple mean, i.e. testing that all the parameters are zero except the intercept

Source DF Sum of Squares Mean Square F Ratio

$\begin{array}{lllll}\text { Model } & 2 & 0.00002785 & 0.000014 & 0.0544\end{array}$

$\begin{array}{lllll}\text { Error } & 17 & 0.00435524 & 0.000256 & \text { Prob > F }\end{array}$

$\begin{array}{llll}\text { C. Total } & 19 & 0.00438309 & 0.9473\end{array}$

\section{Lack Of Fit}

Using replicated points as the part of residual error that does not depend on the form of the model so that you can test for the adequacy of the form of the model.

Source

Lack Of Fit

Pure Error

Sum of Squares
1 $\quad 0.00018605$

Mean Square 0.000186 0.00416919

0.000261

F Ratio

0.7140

Total Error $\quad 17 \quad 0.00435524$

0.4106

Max RSq

0.0488

Parameter Estimates

Term

Intercept

Sample Type[32]

Calibration[1]

Effect Tests

Source

Sample Type

Calibration

\section{Estimate} 0.49956

$-0.00118$

0.00001
Std Error 0.003579

$.003579-0.33$

$0.003579 \quad 0.00$
Prob $>|t|$

$<.0001$

0.7457

0.9978

$\begin{array}{rrrrr}\text { Nparm } & \text { DF } & \text { Sum of Squares } & \text { F Ratio } & \text { Prob > F } \\ 1 & 1 & 0.00002785 & 0.1087 & 0.7457 \\ 1 & 1 & 0.00000000 & 0.0000 & 0.9978\end{array}$


WSRC-TR-2001-00565

Revision 0

Page 28 of 83

November 26, 2001

\section{Appendix (continued)}

\section{Exhibit A3. Statistical Analyses of the Elemental Concentrations as Wet Weight Percents (wt\%'s) of Slurry by Type of Composite and ICP Calibration Block (continued)}

\section{Response Fe}

Whole Model

Actual by Predicted Plot



Residual by Predicted Plot



Summary of Fit

R-square is the portion of variation attributed to the model, between 0 and 1. Root Mean Squared Error "RMSE" estimates the standard deviation of the residual.
RSquare
0.274782
RSquare Adj
0.189463
Root Mean Square Error
0.245995
Mean of Response
5.136935
Observations (or Sum Wgts)
20

Analysis of Variance

The test that the whole model fits better than a simple mean, i.e. testing that all the parameters are zero except the intercept

Source DF Sum of Squares Mean Square F Ratio

$\begin{array}{lllll}\text { Model } & 2 & 0.3897835 & 0.194892 & 3.2206\end{array}$

$\begin{array}{lllll}\text { Error } & 17 & 1.0287333 & 0.060514 & \text { Prob > F }\end{array}$

$\begin{array}{llll}\text { C. Total } & 19 & 1.4185168 & 0.0652\end{array}$

\section{Lack Of Fit}

Using replicated points as the part of residual error that does not depend on the form of the model so that you can test for the adequacy of the form of the model.

Source

Lack Of Fit

Pure Error $\quad 16 \quad 0.9873919$

$\begin{array}{rr}\text { DF } & \text { Sum of Squares } \\ 1 & 0.0413413\end{array}$

Mean Square 0.041341

F Ratio

0.061712 Prob

Prob $>$ F
0.4251

Max RSq

0.3039

Parameter Estimates

Term

Intercept

Sample Type[32]

Calibration[1]

Effect Tests

Source

Sample Type

Calibration
Estimate

5.136935

0.004685

0.139525

Nparm

DF Sum of Squares

Std Error 0.055006 0.055006

0.055006

t Ratio

93.39

Prob $>|t|$

$<.0001$

$0.09 \quad 0.9331$

$2.54 \quad 0.0213$

$\begin{array}{rrrrr}\text { Nparm } & \text { DF } & \text { Sum of Squares } & \text { F Ratio } & \text { Prob > F } \\ 1 & 1 & 0.00043898 & 0.0073 & 0.9331 \\ 1 & 1 & 0.38934451 & 6.4340 & 0.0213\end{array}$


WSRC-TR-2001-00565

Revision 0

Page 29 of 83

November 26, 2001

\section{Appendix (continued)}

\section{Exhibit A3. Statistical Analyses of the Elemental Concentrations as Wet Weight Percents (wt\%'s) of Slurry by Type of Composite and ICP Calibration Block (continued)}

\section{Response Mg}

Whole Model

Actual by Predicted Plot

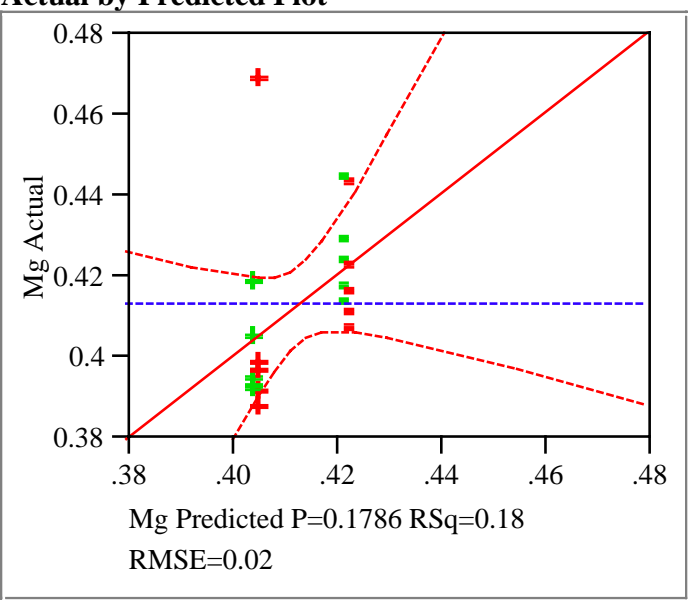

Residual by Predicted Plot

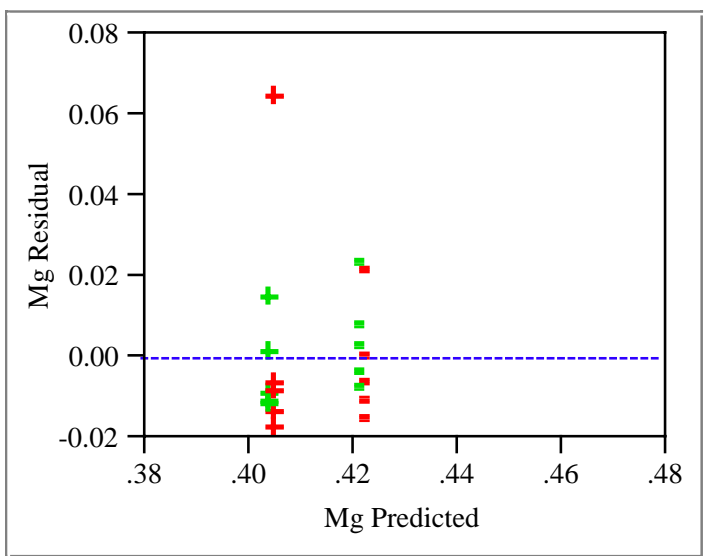

\section{Summary of Fit}

R-square is the portion of variation attributed to the model, between 0 and 1. Root Mean Squared Error "RMSE" estimates the standard deviation of the residual.
RSquare
0.183432
RSquare Adj
0.087366
Root Mean Square Error
0.020021
Mean of Response
0.41315
Observations (or Sum Wgts)
20
Analysis of Variance

The test that the whole model fits better than a simple mean, i.e. testing that all the parameters are zero except the intercept

Source DF Sum of Squares Mean Square F Ratio

$\begin{array}{lllll}\text { Model } & 2 & 0.00153076 & 0.000765 & 1.9094\end{array}$

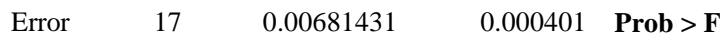

$\begin{array}{llll}\text { C. Total } & 19 & 0.00834507 & 0.1786\end{array}$

\section{Lack Of Fit}

Using replicated points as the part of residual error that does not depend on the form of the model so that you can test for the adequacy of the form of the model.

Source

$$
\begin{array}{lrrrr}
\text { Source } & \text { DF } & \text { Sum of Squares } & \text { Mean Square } & \text { F Ratio } \\
\text { Lack Of Fit } & 1 & 0.00022445 & 0.000224 & 0.5450 \\
\text { Pure Error } & 16 & 0.00658986 & 0.000412 & \text { Prob > F } \\
\text { Total Error } & 17 & 0.00681431 & & 0.4711 \\
& & & & \text { Max RSq } \\
& & & & 0.2103
\end{array}
$$$$
\begin{array}{lllr}
\text { Total Error } & 17 & 0.00681431 & 0.4711
\end{array}
$$

Parameter Estimates

Term

Intercept

Sample Type[32]

Calibration[1]

Effect Tests

Source

Sample Type

Calibration

$$
\begin{array}{rrrr}
\text { Estimate } & \text { Std Error } & \mathbf{t} \text { Ratio } & \text { Prob }>|\mathbf{t}| \\
0.41315 & 0.004477 & 92.29 & <.0001 \\
0.00057 & 0.004477 & 0.13 & 0.9002
\end{array}
$$

$\begin{array}{llll}0.00873 & 0.004477 & 1.95 & 0.0679\end{array}$ 
WSRC-TR-2001-00565

Revision 0

Page 30 of 83

November 26, 2001

\section{Appendix (continued)}

\section{Exhibit A3. Statistical Analyses of the Elemental Concentrations as Wet Weight Percents (wt\%'s) of Slurry by Type of Composite and ICP Calibration Block (continued)}

\section{Response Mn}

Whole Model

Actual by Predicted Plot

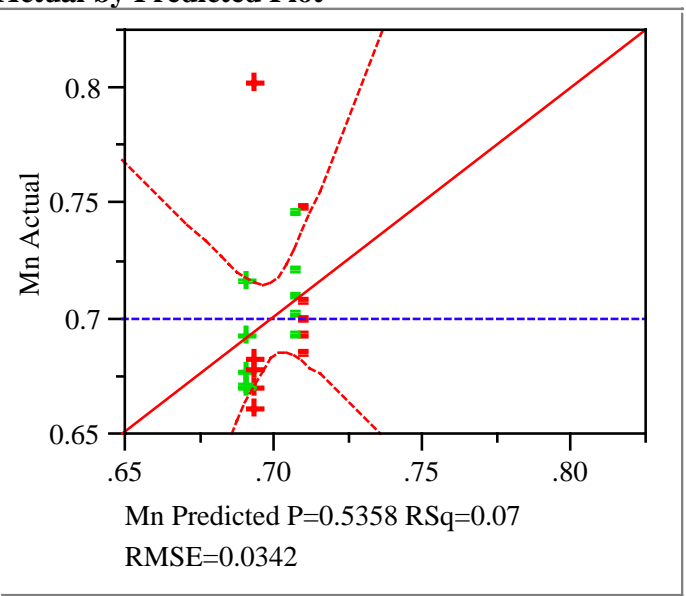

Residual by Predicted Plot

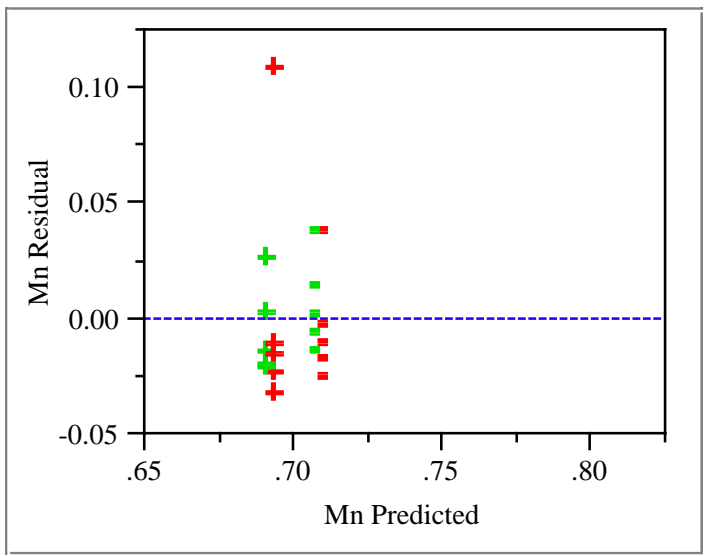

Summary of Fit

R-square is the portion of variation attributed to the model, between 0 and 1. Root Mean Squared Error "RMSE" estimates the standard deviation of the residual.
RSquare
0.07078
RSquare Adj
$-0.03854$
Root Mean Square Error 0.034165
Mean of Response 0.70026
Observations (or Sum Wgts)
Analysis of Variance

The test that the whole model fits better than a simple mean, i.e. testing that all the parameters are zero except the intercept

Source DF Sum of Squares Mean Square F Ratio

$\begin{array}{lllll}\text { Model } & 2 & 0.00151153 & 0.000756 & 0.6475\end{array}$

$\begin{array}{lllll}\text { Error } & 17 & 0.01984364 & 0.001167 & \text { Prob > F }\end{array}$

$\begin{array}{llll}\text { C. Total } & 19 & 0.02135517 & 0.5358\end{array}$

\section{Lack Of Fit}

Using replicated points as the part of residual error that does not depend on the form of the model so that you can test for the adequacy of the form of the model.

Source DF Sum of Squares Mean Square F Ratio

Source DF Sum of Squares Mean Square F Ratio

$\begin{array}{lrrrr}\text { Lack Of Fit } & 1 & 0.00052839 & 0.000528 & 0.4377\end{array}$

$\begin{array}{lllll}\text { Pure Error } & 16 & 0.01931525 & 0.001207 & \text { Prob }>\text { F }\end{array}$

$\begin{array}{llrr}\text { Total Error } & 17 & 0.01984364 & 0.5177\end{array}$

Max RSq

0.0955

Parameter Estimates

Term

Intercept

Sample Type[32]

Calibration[1]

Effect Tests

Source

Sample Type

Calibration

$\begin{array}{rrrr}\text { Estimate } & \text { Std Error } & \text { t Ratio } & \text { Prob }>|\mathbf{t}| \\ 0.70026 & 0.00764 & 91.66 & <.0001 \\ 0.0014 & 0.00764 & 0.18 & 0.8568 \\ 0.00858 & 0.00764 & 1.12 & 0.2770\end{array}$

Nparm

$\begin{array}{rrrrr} & \text { DF } & \text { Sum of Squares } & \text { F Ratio } & \text { Prob > F } \\ 1 & 1 & 0.00003920 & 0.0336 & 0.8568 \\ 1 & 1 & 0.00147233 & 1.2613 & 0.2770\end{array}$


WSRC-TR-2001-00565

Revision 0

Page 31 of 83

November 26, 2001

\section{Appendix (continued)}

\section{Exhibit A3. Statistical Analyses of the Elemental Concentrations as Wet Weight Percents (wt\%'s) of Slurry by Type of Composite and ICP Calibration Block (continued)}

\section{Response Na}

Whole Model

Actual by Predicted Plot

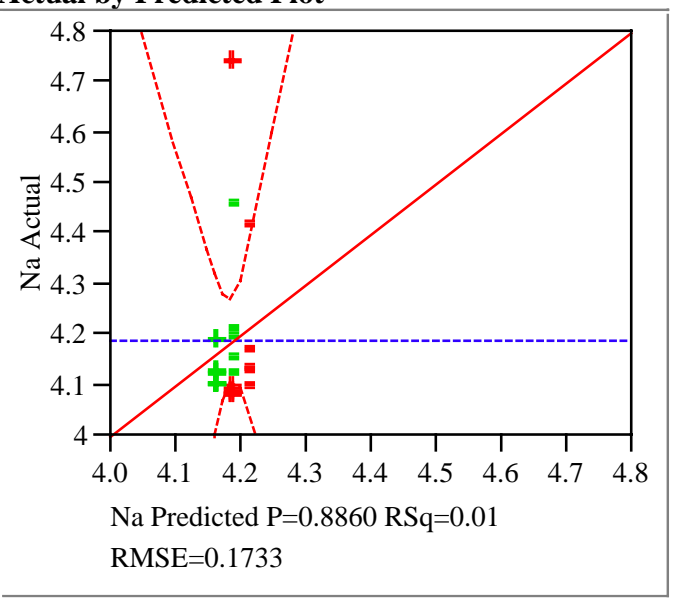

Residual by Predicted Plot



Summary of Fit

R-square is the portion of variation attributed to the model, between 0 and 1. Root Mean Squared Error "RMSE" estimates the standard deviation of the residual.

RSquare 0.014135

RSquare Adj $\quad-0.10185$

Root Mean Square Error $\quad 0.173337$

Mean of Response $\quad 4.18738$

Observations (or Sum Wgts) 20

Analysis of Variance

The test that the whole model fits better than a simple mean, i.e. testing that all the parameters are zero except the intercept

Source DF Sum of Squares Mean Square F Ratio

$\begin{array}{lllll}\text { Model } & 2 & 0.00732354 & 0.003662 & 0.1219\end{array}$

$\begin{array}{lllll}\text { Error } & 17 & 0.51077977 & 0.030046 & \text { Prob > F }\end{array}$

$\begin{array}{llll}\text { C. Total } & 19 & 0.51810331 & 0.8860\end{array}$

\section{Lack Of Fit}

Using replicated points as the part of residual error that does not depend on the form of the model so that you can test for the adequacy of the form of the model.

Source

Lack Of Fit

Pure Error

DF $\quad$ Sum of Squares

Mean Square 0.020390 0.49038928

Total Error 0.51077977

0.030649

F Ratio

0.6653

Prob > F

0.4267

Max RSq

0.0535

Parameter Estimates

Term

Intercept

Sample Type[32]

Calibration[1]

Effect Tests

Source

Sample Type

Calibration

\section{Estimate}

4.18738

0.01213

0.0148

Nparm
DF Sum of Squares

$\begin{array}{rrr}1 & 1 & 0.00294274\end{array}$
Std Error

$0.038759 \quad 0.31$

0.38

Prob> $|\mathbf{t}|$

$<.0001$

0.7581

0.7073 
WSRC-TR-2001-00565

Revision 0

Page 32 of 83

November 26, 2001

\section{Appendix (continued)}

\section{Exhibit A3. Statistical Analyses of the Elemental Concentrations as Wet Weight Percents (wt\%'s) of Slurry by Type of Composite and ICP Calibration Block (continued)}

Response U

Whole Model

Actual by Predicted Plot

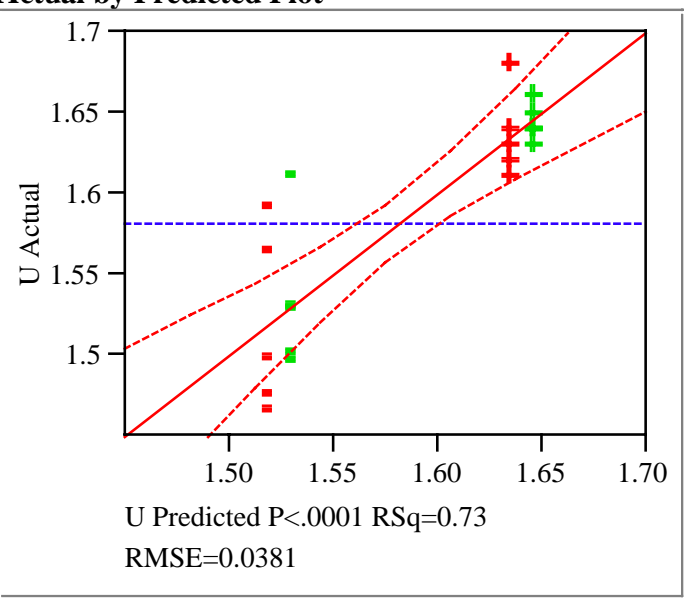

Residual by Predicted Plot

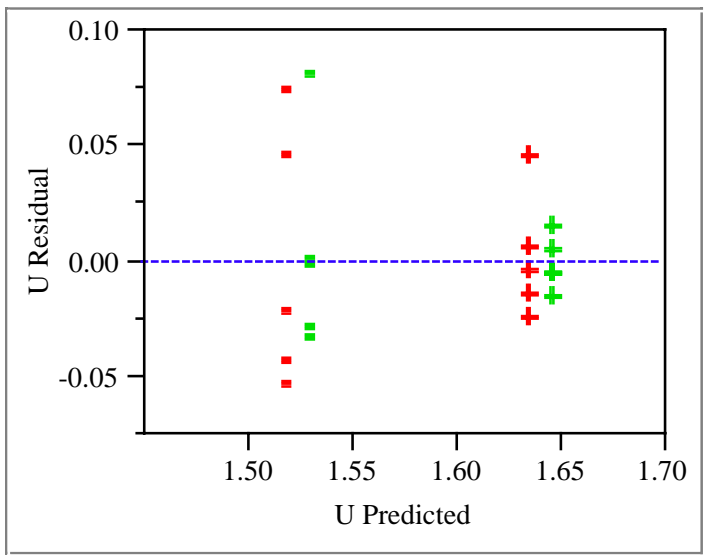

Summary of Fit

R-square is the portion of variation attributed to the model, between 0 and 1. Root Mean Squared Error "RMSE" estimates the standard deviation of the residual.
RSquare
0.734085
RSquare Adj
0.702801
Root Mean Square Error
0.038068
Mean of Response
1.581955
Observations (or Sum Wgts)
20

Analysis of Variance

The test that the whole model fits better than a simple mean, i.e. testing that all the parameters are zero except the intercept

Source DF Sum of Squares Mean Square F Ratio

$\begin{array}{lllll}\text { Model } & 2 & 0.06800829 & 0.034004 & 23.4651\end{array}$

$\begin{array}{lllll}\text { Error } & 17 & 0.02463530 & 0.001449 & \text { Prob }>\text { F }\end{array}$

$\begin{array}{llll}\text { C. Total } & 19 & 0.09264359 & <.0001\end{array}$

\section{Lack Of Fit}

Using replicated points as the part of residual error that does not depend on the form of the model so that you can test for the adequacy of the form of the model.

Source

DF Sum of Squares Mean Square

Lack Of Fit $\quad 1 \quad 0.00005024$

Pure Error $\quad 16 \quad 0.02458506$

Total Error $\quad 17 \quad 0.02463530$

$0.000050 \quad 0.0327$

0.001537 Prob > F

0.8588

Max RSq

0.7346

Parameter Estimates

Term

Estimate Std Error t Ratio Prob $>|\mathbf{t}|$

$\begin{array}{llrrr}\text { Intercept } \quad 1.581955 & 0.008512 & 185.85 & <.0001\end{array}$

$\begin{array}{lllll}\text { Sample Type[32] } & -0.005585 & 0.008512 & -0.66 & 0.5205\end{array}$

$\begin{array}{lllll}\text { Calibration[1] } & -0.058045 & 0.008512 & -6.82 & <.0001\end{array}$

Effect Tests

Source

Nparm

DF Sum of Squares

Sample Type

Calibration

$\begin{array}{ll}1 & 1 \\ 1 & 1\end{array}$

0.00062384

F Ratio Prob > F

$$
\begin{array}{lr}
0.06738444 & 46.4997
\end{array}
$$

0.5205

$<.0001$ 
WSRC-TR-2001-00565

Revision 0

Page 33 of 83

November 26, 2001

\section{Appendix (continued)}

\section{Exhibit A3. Statistical Analyses of the Elemental Concentrations as Wet Weight Percents (wt\%'s) of Slurry by Type of Composite and ICP Calibration Block (continued)}

\section{Response Fe/Na}

Whole Model

Actual by Predicted Plot

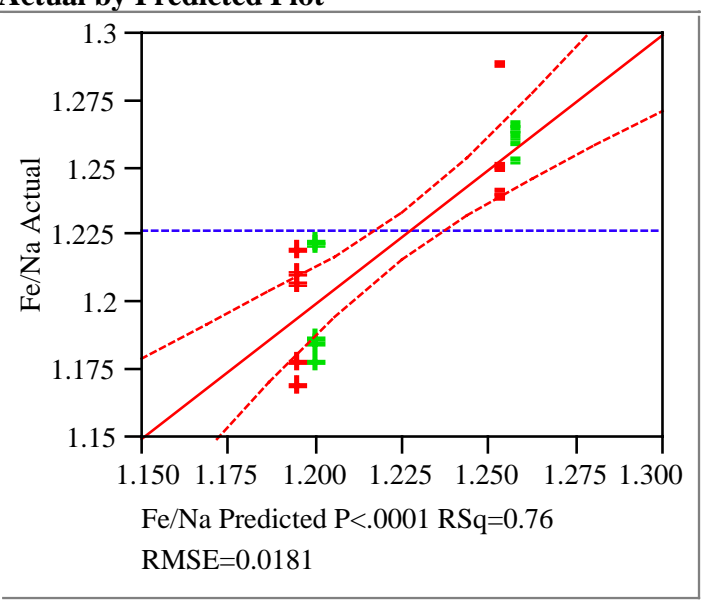

Residual by Predicted Plot

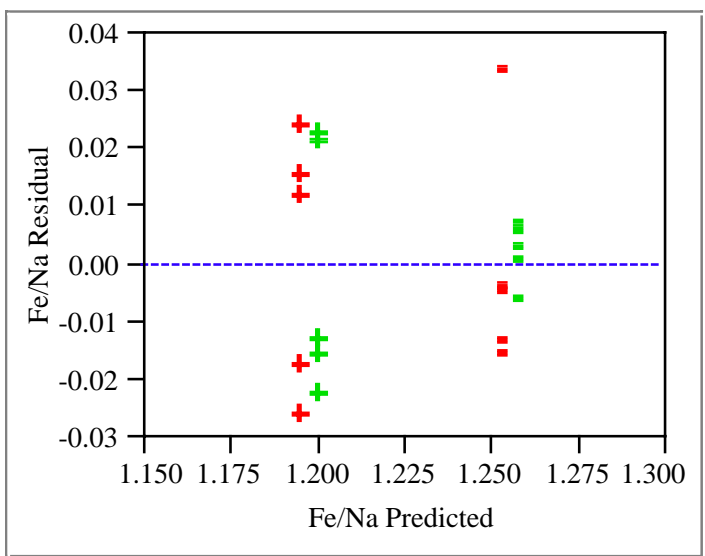

Summary of Fit

R-square is the portion of variation attributed to the model, between 0 and 1. Root Mean Squared Error "RMSE" estimates the standard deviation of the residual.
RSquare
0.755364
RSquare Adj
0.726583
Root Mean Square Error $\quad 0.018054$
Mean of Response $\quad 1.226475$
Observations (or Sum Wgts) 20
Analysis of Variance

The test that the whole model fits better than a simple mean, i.e. testing that all the parameters are zero except the intercept

Source DF Sum of Squares Mean Square F Ratio

$\begin{array}{lllll}\text { Model } & 2 & 0.01710939 & 0.008555 & 26.2455\end{array}$

$\begin{array}{lllll}\text { Error } & 17 & 0.00554113 & 0.000326 & \text { Prob }>\text { F }\end{array}$

$\begin{array}{llll}\text { C. Total } & 19 & 0.02265053 & <.0001\end{array}$

\section{Lack Of Fit}

Using replicated points as the part of residual error that does not depend on the form of the model so that you can test for the adequacy of the form of the model.

Source

Lack Of Fit

Pure Error

DF Sum of Squares 0.00003955
0.00550159

Mean Square

F Ratio $0.000040 \quad 0.1150$

Total Error

0.00554113

0.000344

Prob > F

0.7389

Max RSq

0.7571

Parameter Estimates

Term

Intercept

Sample Type[32]

Calibration[1]

Effect Tests

Source

Sample Type

Calibration

$\begin{array}{rrrr}\text { Estimate } & \text { Std Error } & \text { t Ratio } & \text { Prob>|t| } \\ 1.2264751 & 0.004037 & 303.81 & <.0001 \\ -0.002345 & 0.004037 & -0.58 & 0.5690 \\ 0.0291543 & 0.004037 & 7.22 & <.0001\end{array}$

$\begin{array}{rrrrr}\text { Nparm } & \text { DF } & \text { Sum of Squares } & \text { F Ratio } & \text { Prob > F } \\ 1 & 1 & 0.00010996 & 0.3374 & 0.5690 \\ 1 & 1 & 0.01699943 & 52.1536 & <.0001\end{array}$ 
WSRC-TR-2001-00565

Revision 0

Page 34 of 83

November 26, 2001

\section{Appendix (continued)}

\section{Exhibit A4. Sensitivity of Statistical Analyses of the Elemental Concentrations as Wet Weight Percents (wt\%'s) of Slurry to 5\% Differences Due to Type of Composite}

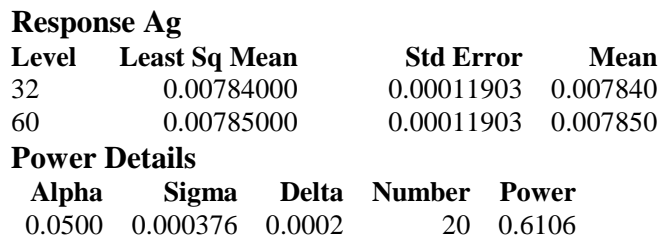

Response Al

\begin{tabular}{|c|c|c|c|c|}
\hline Level & \multicolumn{2}{|c|}{ Least Sq Mean } & Std Error & Mean \\
\hline 32 & \multicolumn{2}{|c|}{1.3878600} & 0.01970206 & 1.38786 \\
\hline 60 & \multicolumn{2}{|c|}{1.3877400} & 0.01970206 & 1.38774 \\
\hline \multicolumn{5}{|c|}{ Power Details } \\
\hline Alpha & Sigma & Delta & Number & Power \\
\hline 0.0500 & 0.062303 & 0.03469 & 20 & 0.6509 \\
\hline
\end{tabular}

Response Ca

\begin{tabular}{|c|c|c|c|c|}
\hline Level & \multicolumn{2}{|c|}{ Least Sq Mean } & Std Error & Mean \\
\hline 32 & \multicolumn{2}{|c|}{0.49838000} & 0.0050615 & 0.498380 \\
\hline 60 & \multicolumn{2}{|c|}{0.50074000} & 0.0050615 & 0.500740 \\
\hline \multicolumn{5}{|c|}{ Power Details } \\
\hline Alpha & Sigma & Delta & Number & Power \\
\hline 0.0500 & 0.016006 & 0.01252 & 20 & 0.9090 \\
\hline
\end{tabular}

\section{Response Fe}

Level Least Sq Mean

$32 \quad 5.1416200$

$60 \quad 5.1322500$

Power Details

Alpha Sigma Delta Number Power

$\begin{array}{lllll}0.0500 & 0.245995 & 0.12831 & 20 & 0.5948\end{array}$

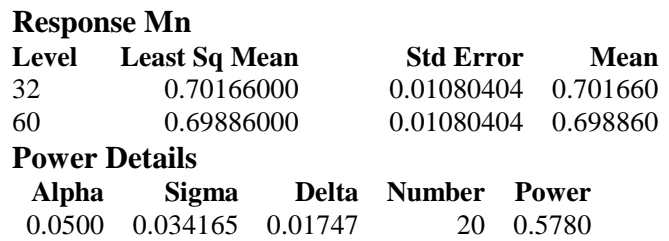

Response Na



$\begin{array}{llll}60 & 4.1752500 & 0.05481411 & 4.17525\end{array}$

Power Details

$\begin{array}{rrrrr}\text { Alpha } & \text { Sigma } & \text { Delta } & \text { Number } & \text { Power } \\ 0.0500 & 0.173337 & 0.10438 & 20 & 0.7186\end{array}$

Response U

$\begin{array}{lrrr}\text { Level } & \text { Least Sq Mean } & \text { Std Error } & \text { Mean } \\ 32 & 1.5763700 & 0.01203800 & 1.57637 \\ 60 & 1.5875400 & 0.01203800 & 1.58754\end{array}$

Power Details

Alpha Sigma Delta Number Power

$\begin{array}{lrrrr}0.0500 & 0.038068 & 0.03969 & 20 & 0.9924\end{array}$

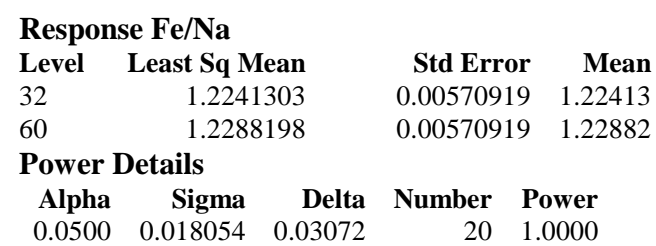

\begin{tabular}{|c|c|c|c|c|}
\hline \multicolumn{5}{|c|}{ Response Mg } \\
\hline Level & Least Sq $\mathrm{N}$ & ean & Std Erro & Mean \\
\hline 32 & 0.4137 & 000 & 0.0063312 & 0.413720 \\
\hline 60 & 0.4125 & 000 & 0.0063312 & 0.412580 \\
\hline \multicolumn{5}{|c|}{ Power Details } \\
\hline Alpha & Sigma & Delta & Number & Power \\
\hline 0.0500 & 0.020021 & 0.01031 & 20 & 0.5839 \\
\hline
\end{tabular}


WSRC-TR-2001-00565

Revision 0

Page 35 of 83

November 26, 2001

\section{Appendix (continued)}

\section{Exhibit A5. Statistical Analyses of the Elemental Concentrations as Wet Weight Percents (wt\%'s) of Slurry with Potential Outliers Removed by Type of Composite and ICP Calibration Block}

\section{Response Ag}

\section{LIMS Numbers Removed From this Analysis:} 300166387 and 300166390 .

Actual by Predicted Plot

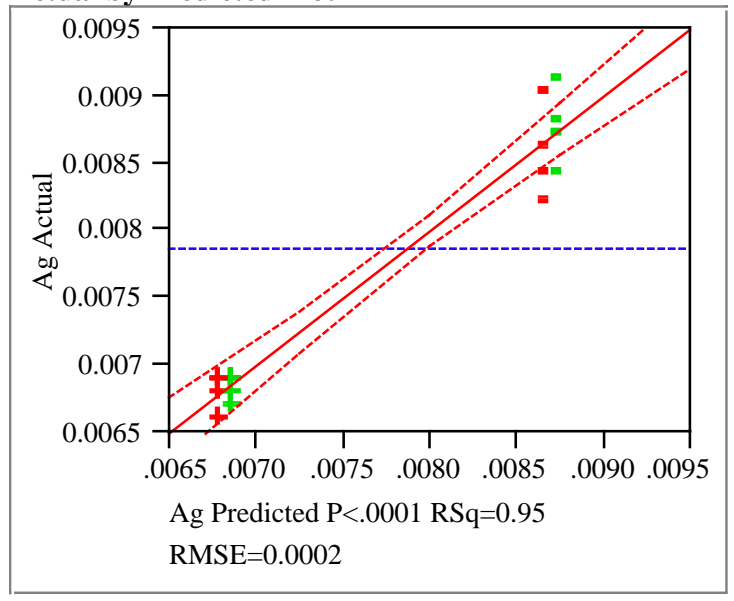

\section{Summary of Fit}

RSquare $\quad 0.948155$

RSquare Adj $\quad 0.941242$

Root Mean Square Error $\quad 0.000239$

Mean of Response $\quad 0.007856$

Observations (or Sum Wgts) 18

Analysis of Variance

Source DF Sum of Squares Mean Square F Ratio

$\begin{array}{lllll}\text { Model } & 2 & 0.00001569 & 0.0000078 & 137.1614\end{array}$

$\begin{array}{lllll}\text { Error } & 15 & 0.00000086 & 5.7183 \mathrm{e}-8 & \text { Prob }>\text { F }\end{array}$

$\begin{array}{llll}\text { C. Total } & 17 & 0.00001654 & <.0001\end{array}$

Lack Of Fit

Source DF Sum of Squares Mean Square F Ratio

Lack Of Fit 1

$\begin{array}{lllll}\text { Pure Error } & 14 & 0.00000085 & 6.0821 \mathrm{e}-8 & \text { Prob }>\text { F }\end{array}$

$\begin{array}{llll}\text { Total Error } & 15 & 0.00000086 & 0.7533\end{array}$

Max RSq

0.9485

Parameter Estimates

Term

Intercept

Sample Type[32]

Calibration[1]

Effect Tests

Source

Sample Type

Calibration
Estimate Std Error t Ratio Prob $>|\mathbf{t}|$

$\begin{array}{llll}0.0077512 & 0.000057 & 136.67 & <.0001\end{array}$

$\begin{array}{llll}-0.000033 & 0.000056 & -0.59 & 0.5631\end{array}$

$\begin{array}{llll}0.0009387 & 0.000057 & 16.55 & <.0001\end{array}$

Nparm DF Sum of Squares F Ratio Prob > F $0.00000002 \quad 0.3498 \quad 0.5631$ $0.00001567 \quad 273.9731 \quad<.0001$
Residual by Predicted Plot



Sample Type

Least Squares Means Table

$\begin{array}{lrrr}\text { Level } & \text { Least Sq Mean } & \text { Std Error } & \text { Mean } \\ 32 & 0.00771792 & 0.00007996 & 0.007822 \\ 60 & 0.00778458 & 0.00007996 & 0.007889\end{array}$

Power Details

Alpha Sigma Delta Number Power

$\begin{array}{lrrrr}0.0500 & 0.000239 & 0.000195 & 18 & 0.8970\end{array}$ 
WSRC-TR-2001-00565

Revision 0

Page 36 of 83

November 26, 2001

\section{Appendix (continued)}

\section{Exhibit A5. Statistical Analyses of the Elemental Concentrations as Wet Weight Percents (wt\%'s) of Slurry with Potential Outliers Removed by Type of Composite and ICP Calibration Block (continued)}

\section{Response Al}

LIMS Numbers Removed From this Analysis: $300166376,300166386,300166387$, and 300166390 .

Actual by Predicted Plot

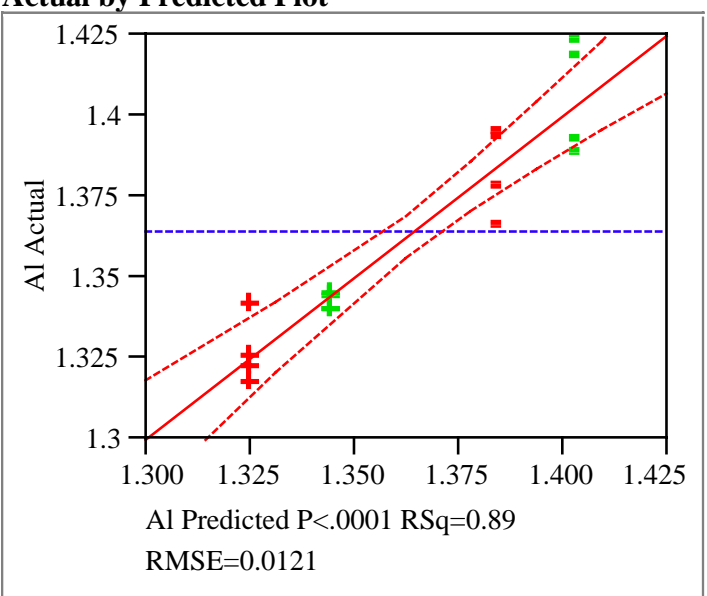

Summary of Fit

RSquare

RSquare Adj

0.88947

0.872465

0.012111

1.363831

Mean of Response

Observations (or Sum Wgts)

Analysis of Variance

Source DF Sum of Squares

$\begin{array}{lll}\text { Model } & 2 & 0.01534461\end{array}$

$\begin{array}{lll}\text { Error } & 13 & 0.00190680\end{array}$

$\begin{array}{lll}\text { C. Total } & 15 & 0.01725141\end{array}$

Lack Of Fit

\section{Source}

Lack Of Fit

DF

Pure Error

12

Sum of Squares 0.00004796 0.00185885

Total Error 0.00190680

\section{Mean Square F Ratio $0.007672 \quad 52.3074$ 0.000147 Prob $>$ F \\ $<.0001$}

Mean Square 0.000048
0.000155

F Ratio 0.3096 Prob > F 0.5882

Max RSq 0.8922

Parameter Estimates

Term

Intercept

$$
\text { Estimate }
$$
1.3638312 $-0.009481$ 0.0294812

Std Error 0.003028 0.003028 0.003028

Calibration[1]

Effect Tests

Source

Sample Type

Calibration

Npar

$\begin{array}{rrrrr}\text { Nparm } & \text { DF } & \text { Sum of Squares } & \text { F Ratio } & \text { Prob > F } \\ 1 & 1 & 0.00143831 & 9.8059 & 0.0080 \\ 1 & 1 & 0.01390631 & 94.8089 & <.0001\end{array}$

Residual by Predicted Plot

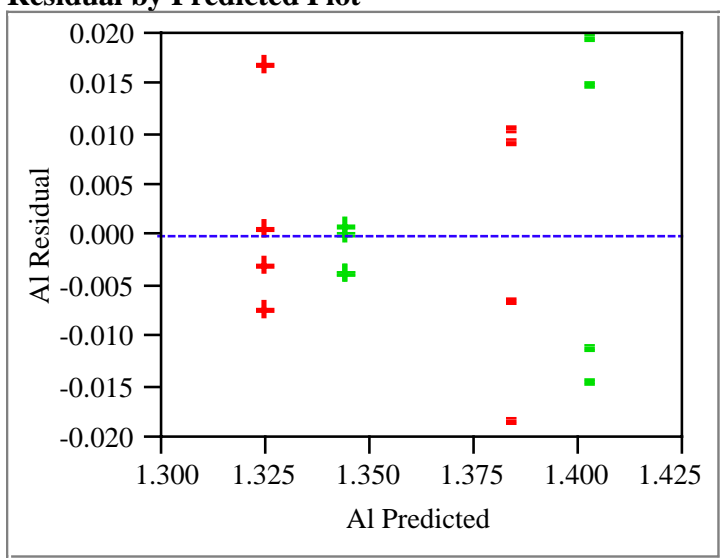

Sample Type

Least Squares Means Table

Level Least Sq Mean Std Error Mean

$\begin{array}{llll}32 & 1.3543500 & 0.00428190 & 1.35435\end{array}$

$\begin{array}{llll}60 & 1.3733125 & 0.00428190 & 1.37331\end{array}$

Power Details

Test

Sample Type

Power

Alpha Sigma Delta Number Power

$\begin{array}{lllll}0.0500 & 0.012111 & 0.034333 & 16 & 1.00000\end{array}$ 
WSRC-TR-2001-00565

Revision 0

Page 37 of 83

November 26, 2001

\section{Appendix (continued)}

\section{Exhibit A5. Statistical Analyses of the Elemental Concentrations as Wet Weight Percents (wt\%'s) of Slurry with Potential Outliers Removed by Type of Composite and ICP Calibration Block (continued)}

\section{Response Ca}

\section{LIMS Numbers Removed From this Analysis:}

\section{$300166376,300166386,300166387$, and 300166390 .}

Actual by Predicted Plot

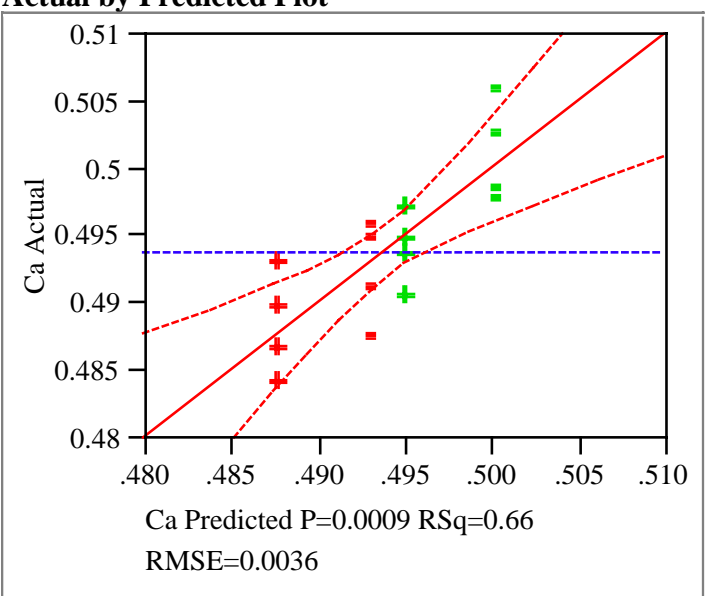

Summary of Fit

RSquare

RSquare Adj

0.661594

0.609531

0.00357

Root Mean Square Error

0.493881

Mean of Response

Observations (or Sum Wgts)

Analysis of Variance

Source DF Sum of Squares

$\begin{array}{lll}\text { Model } & 2 & 0.00032387\end{array}$

$\begin{array}{lll}\text { Error } & 13 & 0.00016566\end{array}$

C. Total $15 \quad 0.00048952$

Lack Of Fit

$\begin{array}{lrr}\text { Source } & \text { DF } & \text { Sum of Squares } \\ \text { Lack Of Fit } & 1 & 0.00001106 \\ \text { Pure Error } & 12 & 0.00015460\end{array}$

Total Error $\quad 13 \quad 0.00016566$

$$
\begin{array}{rr}
\text { Mean Square } & \text { F Ratio } \\
0.000162 & 12.7077 \\
0.000013 & \text { Prob > F } \\
0.0009
\end{array}
$$

$$
\begin{array}{rr}
\text { Mean Square } & \text { F Ratio } \\
0.000011 & 0.8581 \\
0.000013 & \text { Prob > F } \\
& 0.3725 \\
\text { Max RSq } \\
0.6842
\end{array}
$$

Parameter Estimates

$\begin{array}{lrrrrr}\text { Term } & \begin{array}{r}\text { Estimate } \\ \text { Intercept }\end{array} & \begin{array}{r}\text { Std Error } \\ \text { t Ratio }\end{array} & \text { Prob }>|\mathbf{t}| \\ \text { Sample Type[32] } & 0.4938812 & 0.000892 & 553.41 & <.0001 \\ \text { Calibration[1] } & -0.003631 & 0.000892 & -4.07 & 0.0013 \\ \text { Effect Tests } & 0.0026562 & 0.000892 & 2.98 & 0.0107 \\ \text { Source } & \text { Nparm } & \text { DF } & \text { Sum of Squares } & \text { F Ratio } & \text { Prob > F } \\ \text { Sample Type } & 1 & 1 & 0.00021098 & 16.5563 & 0.0013 \\ \text { Calibration } & 1 & 1 & 0.00011289 & 8.8591 & 0.0107\end{array}$

Residual by Predicted Plot

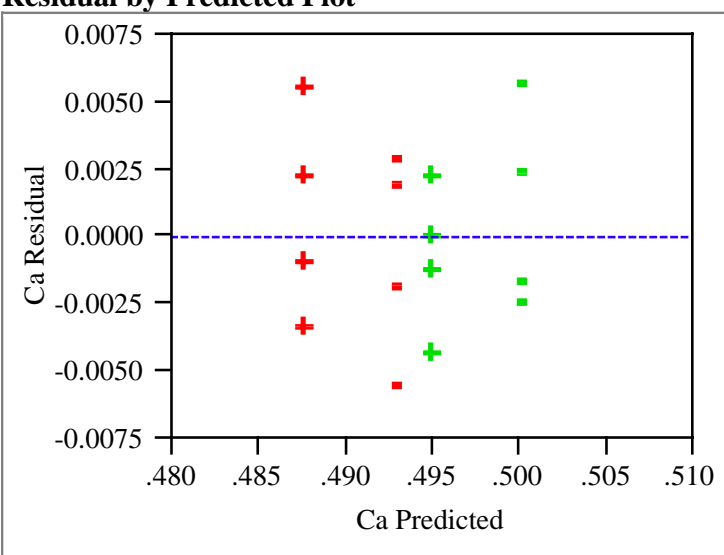

Sample Type

Least Squares Means Table

$\begin{array}{lrrr}\text { Level } & \text { Least Sq Mean } & \text { Std Error } & \text { Mean } \\ 32 & 0.49025000 & 0.00126209 & 0.490250 \\ 60 & 0.49751250 & 0.00126209 & 0.497512\end{array}$

Power Details

Alpha Sigma Delta Number Power $\begin{array}{lrrrr}0.0500 & 0.00357 & 0.012438 & 16 & 1.0000\end{array}$ 
WSRC-TR-2001-00565

Revision 0

Page 38 of 83

November 26, 2001

\section{Appendix (continued)}

\section{Exhibit A5. Statistical Analyses of the Elemental Concentrations as Wet Weight Percents (wt\%'s) of Slurry with Potential Outliers Removed by Type of Composite and ICP Calibration Block (continued)}

\section{Response Fe}

\section{LIMS Numbers Removed From this Analysis:} 300166386 and 300166390.

Actual by Predicted Plot

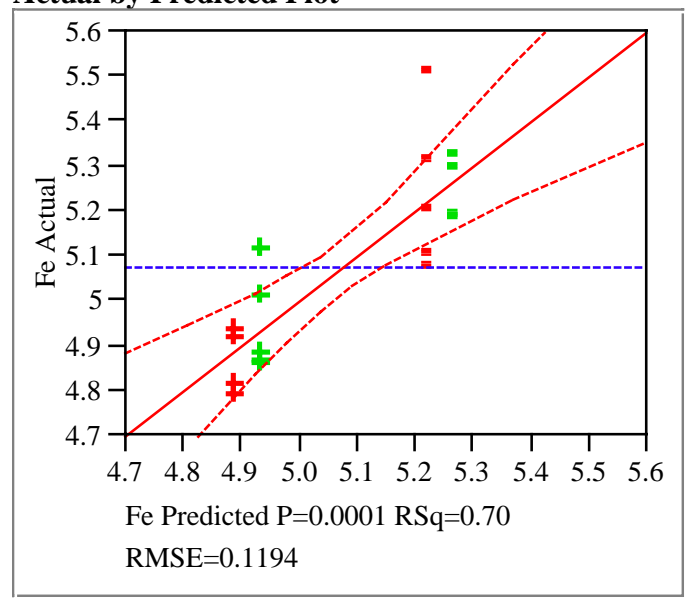

\section{Summary of Fit}

RSquare $\quad 0.698199$

RSquare Adj $\quad 0.657958$

Root Mean Square Error $\quad 0.119414$

Mean of Response $\quad 5.074756$

Observations (or Sum Wgts) 18

Analysis of Variance

Source DF Sum of Squares Mean Square F Ratio

$\begin{array}{lrrrr}\text { Model } & 2 & 0.49483049 & 0.247415 & 17.3508 \\ \text { Error } & 15 & 0.21389415 & 0.014260 & \text { Prob > F }\end{array}$

$\begin{array}{llrrr}\text { Error } & 15 & 0.21389415 & 0.014260 & \text { Prob > F } \\ \text { C. Total } & 17 & 0.70872464 & & 0.0001\end{array}$

Lack Of Fit

Source DF Sum of Squares

Lack Of Fit $\quad 1 \quad 0.00628588$

Pure Error $\quad 14 \quad 0.20760827$

Total Error $\quad 15 \quad 0.21389415$

$$
\begin{array}{rr}
\text { Mean Square } & \text { F Ratio } \\
0.006286 & 0.4239 \\
0.014829 & \text { Prob }>\text { F } \\
& 0.5255 \\
\text { Max RSq }
\end{array}
$$

Parameter Estimates

Term

Intercept

Sample Type[32]

Calibration[1]

Effect Tests

Source

Sample Type

Calibration

$$
\begin{array}{rrrrrr} 
& \text { Estimate } & \text { Std Error } & \text { t Ratio } & \text { Prob }>|\mathbf{t}| \\
& 5.0747556 & 0.028146 & 180.30 & <.0001 \\
& -0.022576 & 0.028321 & -0.80 & 0.4378 \\
& 0.1667862 & 0.028321 & 5.89 & <.0001 \\
\text { Nparm } & \text { DF } & \text { Sum of Squares } & \text { F Ratio } & \text { Prob > F } \\
1 & 1 & 0.00906110 & 0.6354 & 0.4378 \\
1 & 1 & 0.49453606 & 34.6809 & <.0001
\end{array}
$$

Least Squares Means Table

$\begin{array}{lrrr}\text { Level } & \text { Least Sq Mean } & \text { Std Error } & \text { Mean } \\ 32 & 5.0521793 & 0.03992873 & 5.07071 \\ 60 & 5.0973318 & 0.03992873 & 5.07880\end{array}$

Power Details

$\begin{array}{rrrrr}\text { Alpha } & \text { Sigma } & \text { Delta } & \text { Number } & \text { Power } \\ 0.0500 & 0.119414 & 0.127333 & 18 & 0.9881\end{array}$


WSRC-TR-2001-00565

Revision 0

Page 39 of 83

November 26, 2001

\section{Appendix (continued)}

\section{Exhibit A5. Statistical Analyses of the Elemental Concentrations as Wet Weight Percents (wt\%'s) of Slurry with Potential Outliers Removed by Type of Composite and ICP Calibration Block (continued)}

\section{Response Mg}

\section{LIMS Numbers Removed From this Analysis:} 300166376,300166386 , and 300166390 .

Actual by Predicted Plot

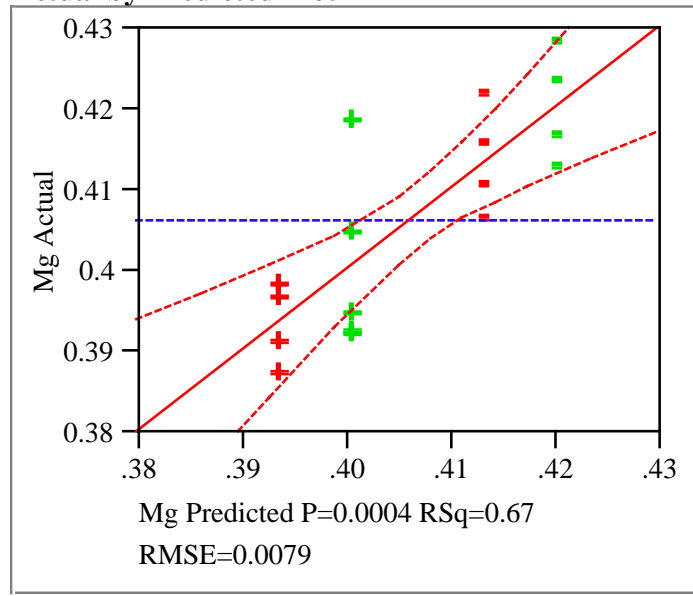

$\begin{array}{ll}\text { Summary of Fit } & \\ \text { RSquare } & 0.673015 \\ \text { RSquare Adj } & 0.626303 \\ \text { Root Mean Square Error } & 0.007875 \\ \text { Mean of Response } & 0.406347\end{array}$

Observations (or Sum Wgts) 17

Analysis of Variance

Source DF Sum of Squares Mean Square F Ratio

$\begin{array}{lllll}\text { Model } & 2 & 0.00178721 & 0.000894 & 14.4077\end{array}$

$\begin{array}{lllll}\text { Error } & 14 & 0.00086831 & 0.000062 & \text { Prob > F }\end{array}$

$\begin{array}{llll}\text { C. Total } & 16 & 0.00265552 & 0.0004\end{array}$

Lack Of Fit

Source DF Sum of Squares

Lack Of Fit $1 \quad 0.00000044$

Pure Error $\quad 13 \quad 0.00086787$

Mean Square F Ratio $\begin{array}{rr}0.000000 & 0.0067\end{array}$

Total Error $\quad 14 \quad 0.00086831$

0.000067 Prob $>$ F 0.9362

Max RSq 0.6732

Parameter Estimates

Term

Intercept

Sample Type[32]

Calibration[1]

Effect Tests

Source

Sample Type

Calibration

$\begin{array}{rrrrr}\text { Nparm } & \text { DF } & \text { Sum of Squares } & \text { F Ratio } & \text { Prob > F } \\ 1 & 1 & 0.00020642 & 3.3282 & 0.0895 \\ 1 & 1 & 0.00163992 & 26.4407 & 0.0001\end{array}$

Residual by Predicted Plot

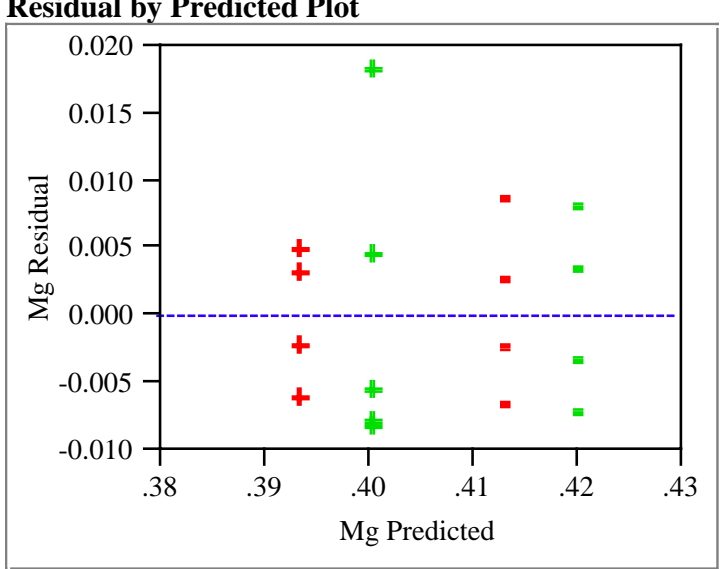

Least Squares Means Table

$\begin{array}{lrrr}\text { Level } & \text { Least Sq Mean } & \text { Std Error } & \text { Mean } \\ 32 & 0.40322500 & 0.00278439 & 0.403225 \\ 60 & 0.41021711 & 0.00263377 & 0.409122\end{array}$

Power Details

$\begin{array}{rrrrr}\text { Alpha } & \text { Sigma } & \text { Delta } & \text { Number } & \text { Power } \\ 0.0500 & 0.007875 & 0.010255 & 17 & 0.9987\end{array}$


WSRC-TR-2001-00565

Revision 0

Page 40 of 83

November 26, 2001

\section{Appendix (continued)}

\section{Exhibit A5. Statistical Analyses of the Elemental Concentrations as Wet Weight Percents (wt\%'s) of Slurry with Potential Outliers Removed by Type of Composite and ICP Calibration Block (continued)}

\section{Response Mn}

\section{LIMS Numbers Removed From this Analysis:} 300166376,300166386 , and 300166390 .

Actual by Predicted Plot
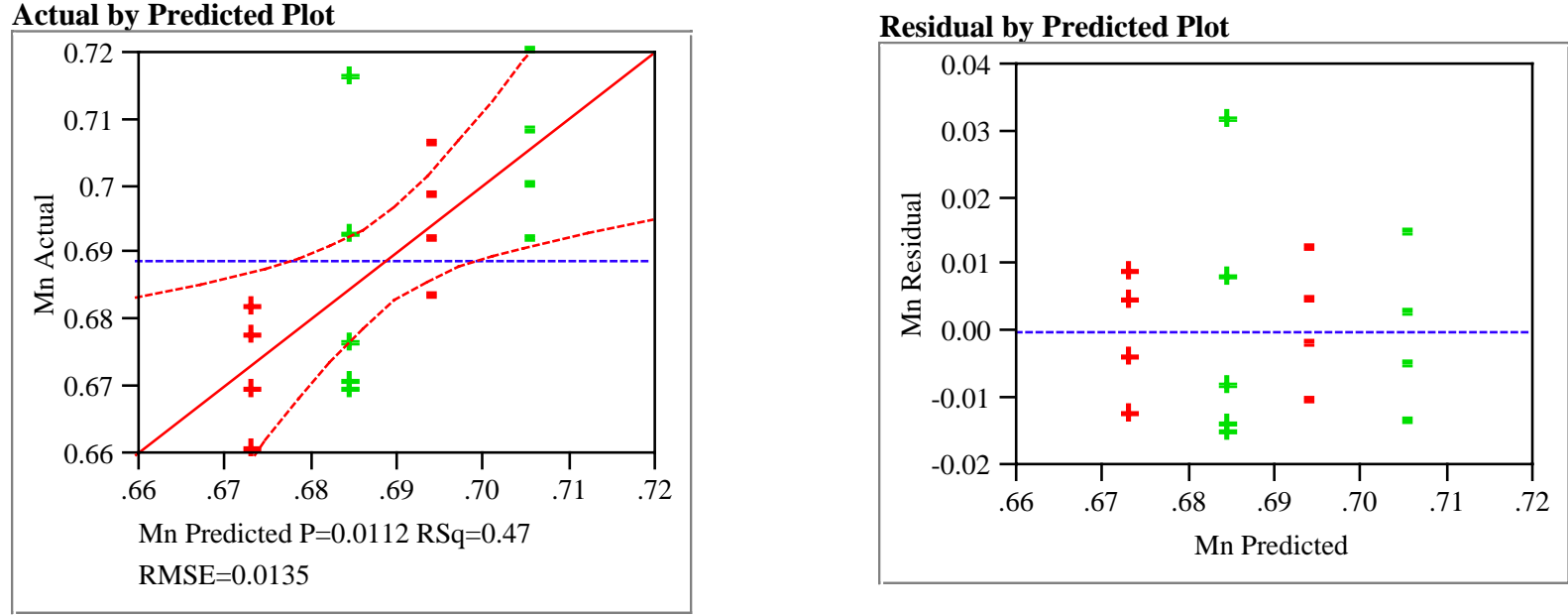

\section{Summary of Fit}

RSquare $\quad 0.473637$

RSquare Adj $\quad 0.398442$

Root Mean Square Error $\quad 0.013497$

Mean of Response $\quad 0.688988$

Observations (or Sum Wgts) 17

Analysis of Variance

Source DF Sum of Squares Mean Square F Ratio

$\begin{array}{lllll}\text { Model } & 2 & 0.00229483 & 0.001147 & 6.2988\end{array}$

$\begin{array}{lllll}\text { Error } & 14 & 0.00255029 & 0.000182 & \text { Prob > F }\end{array}$

$\begin{array}{llll}\text { C. Total } & 16 & 0.00484512 & 0.0112\end{array}$

Lack Of Fit

Source DF Sum of Squares Mean Square F Ratio

$\begin{array}{lllll}\text { Lack Of Fit } & 1 & 0.00000720 & 0.000007 & 0.0368\end{array}$

$\begin{array}{lllll}\text { Pure Error } & 13 & 0.00254309 & 0.000196 & \text { Prob }>\text { F }\end{array}$

$\begin{array}{llll}\text { Total Error } & 14 & 0.00255029 & 0.8508\end{array}$

Max RSq

0.4751

Parameter Estimates

Term

Intercept

Sample Type[32]

Calibration[1]

Effect Tests

Source

Sample Type

Calibration

$\begin{array}{rrrrr}\text { Nparm } & \text { DF } & \text { Sum of Squares } & \text { F Ratio } & \text { Prob > F } \\ 1 & 1 & 0.00056095 & 3.0794 & 0.1011 \\ 1 & 1 & 0.00183967 & 10.0990 & 0.0067\end{array}$

Least Squares Means Table

$\begin{array}{lrrr}\text { Level } & \text { Least Sq Mean } & \text { Std Error } & \text { Mean } \\ 32 & 0.68350000 & 0.00477184 & 0.683500 \\ 60 & 0.69502632 & 0.00451371 & 0.693867\end{array}$

Power Details

$\begin{array}{rrrrr}\text { Alpha } & \text { Sigma } & \text { Delta } & \text { Number } & \text { Power } \\ 0.0500 & 0.013497 & 0.017376 & 17 & 0.9984\end{array}$


WSRC-TR-2001-00565

Revision 0

Page 41 of 83

November 26, 2001

\section{Appendix (continued)}

\section{Exhibit A5. Statistical Analyses of the Elemental Concentrations as Wet Weight Percents (wt\%'s) of Slurry with Potential Outliers Removed by Type of Composite and ICP Calibration Block (continued)}

\section{Response Na}

LIMS Numbers Removed From this Analysis:

300166376,300166386 , and 300166390 .

Actual by Predicted Plot

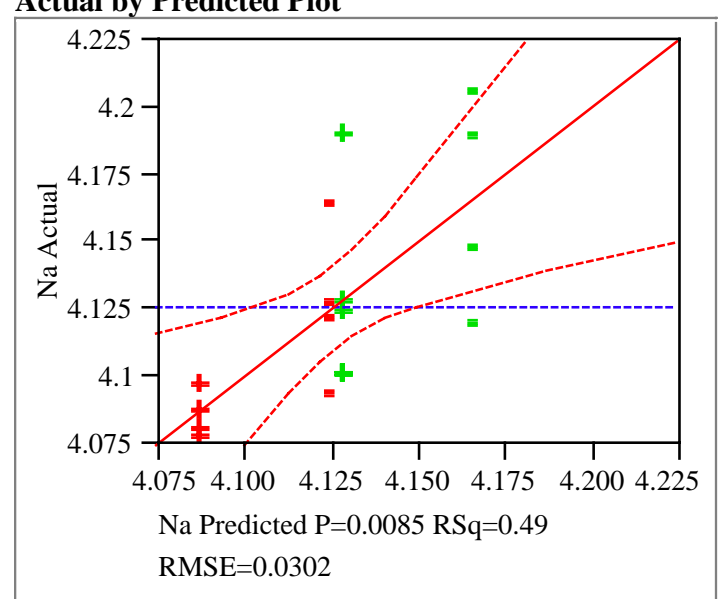

Summary of Fit

RSquare $\quad 0.494149$

RSquare Adj $\quad 0.421884$

Root Mean Square Error $\quad 0.030177$

Mean of Response $\quad 4.125929$

Observations (or Sum Wgts)

Analysis of Variance

Source DF Sum of Squares Mean Square F Ratio

$\begin{array}{lllll}\text { Model } & 2 & 0.01245458 & 0.006227 & 6.8381\end{array}$

$\begin{array}{lllll}\text { Error } & 14 & 0.01274954 & 0.000911 & \text { Prob }>\text { F }\end{array}$

$\begin{array}{llll}\text { C. Total } & 16 & 0.02520412 & 0.0085\end{array}$

Lack Of Fit

Source

Lack Of Fit

Pure Error

$\begin{array}{rr}\text { DF } & \text { Sum of Squares } \\ 1 & 0.00002056\end{array}$

0.01272897

Mean Square 0.000021

0.000979

F Ratio

0.0210

Prob > F 0.8870

$\operatorname{Max} \mathbf{R S q}$

0.4950

Parameter Estimates

Term

Intercept

Sample Type[32]

Calibration[1]

Effect Tests

Source

Sample Type

Calibration
0.01274954

\section{Estimate}

4.1258257

$-0.020588$

0.0188243

Std Error

0.00734

$0.007343-561.86$

$-2.80$

$0.007343 \quad 2.56$

Nparm
Residual by Predicted Plot

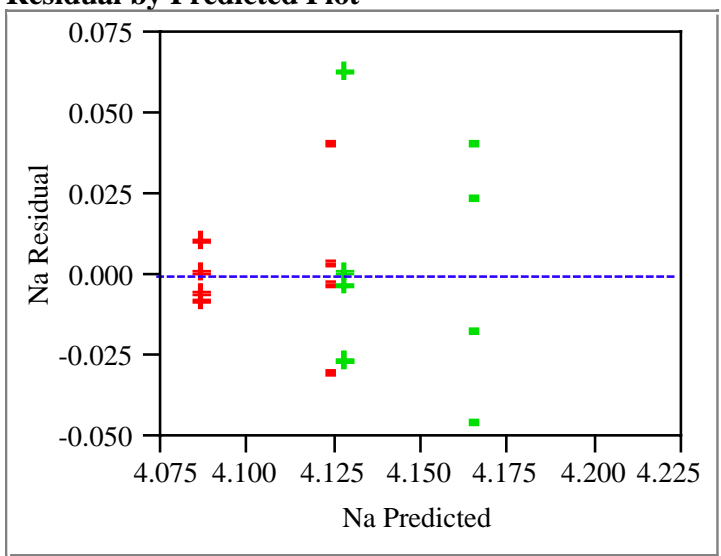

Least Squares Means Table

$\begin{array}{lrrr}\text { Level } & \text { Least Sq Mean } & \text { Std Error } & \text { Mean } \\ 32 & 4.1052375 & 0.01066936 & 4.10524 \\ 60 & 4.1464138 & 0.01009220 & 4.14432\end{array}$

Power Details

Alpha Sigma Delta Number Power $\begin{array}{lllll}0.0500 & 0.030177 & 0.10366 & 17 & 1.0000\end{array}$ 
WSRC-TR-2001-00565

Revision 0

Page 42 of 83

November 26, 2001

\section{Appendix (continued)}

\section{Exhibit A5. Statistical Analyses of the Elemental Concentrations as Wet Weight Percents (wt\%'s) of Slurry with Potential Outliers Removed by Type of Composite and ICP Calibration Block (continued)}

\section{Response U}

\section{LIMS Numbers Removed From this Analysis:}

\section{6 and 300166390.}

Actual by Predicted Plot



Summary of Fit

RSquare $\quad 0.811815$

RSquare Adj $\quad 0.786723$

Root Mean Square Error $\quad 0.03197$

Mean of Response $\quad 1.575039$

Observations (or Sum Wgts) 18

Analysis of Variance

Source DF $\quad$ Sum of Squares Mean Square $\quad$ F Ratio

$\begin{array}{lllll}\text { Model } & 2 & 0.06613889 & 0.033069 & 32.3543\end{array}$

$\begin{array}{lllll}\text { Error } & 15 & 0.01533155 & 0.001022 & \text { Prob }>\text { F }\end{array}$

$\begin{array}{lllll}\text { C. Total } & 17 & 0.08147044 & <.0001\end{array}$

Lack Of Fit

Source

Lack Of Fit

DF Sum of Squares

$\begin{array}{lll} & & 0.00063947\end{array}$

Pure Error $\quad 14 \quad 0.01469208$

Total Error $\quad 15 \quad 0.01533155$

Mean Square 0.000639 0.001049

F Ratio 0.6093

Prob > F 0.4480

Max RSq 0.8197

Parameter Estimates

Term

Intercept

Sample Type[32]

Calibration[1]

Effect Tests

Source

Sample Type

Npar

Npar 1.5750389 $-0.003502$ $-0.060128$

Std Erro

t Ratio Prob $>|\mathbf{t}|$ $\begin{array}{lll}0.007535 & 209.02<.0001\end{array}$ $\begin{array}{lll}0.007582 & -0.46 & 0.6508\end{array}$ $\begin{array}{lll}0.007582 & -7.93 & <.0001\end{array}$

\section{Estimate}



Calibration

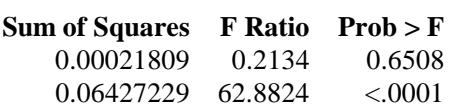

Residual by Predicted Plot

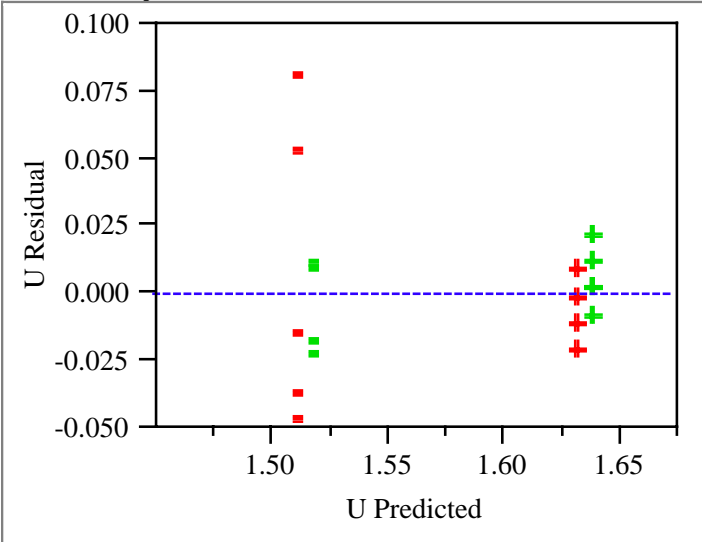

Least Squares Means Table

$\begin{array}{lrrr}\text { Level } & \text { Least Sq Mean } & \text { Std Error } & \text { Mean } \\ 32 & 1.5715364 & 0.01069003 & 1.56486 \\ 60 & 1.5785414 & 0.01069003 & 1.58522\end{array}$

Power Details

$\begin{array}{rrrrr}\text { Alpha } & \text { Sigma } & \text { Delta } & \text { Number } & \text { Power } \\ 0.0500 & 0.03197 & 0.039463 & 18 & 0.9982\end{array}$


WSRC-TR-2001-00565

Revision 0

Page 43 of 83

November 26, 2001

\section{Appendix (continued)}

\section{Exhibit A5. Statistical Analyses of the Elemental Concentrations as Wet Weight Percents (wt\%'s) of Slurry with Potential Outliers Removed by Type of Composite and ICP Calibration Block (continued)}

\section{Response Fe/Na}

\section{LIMS Number Removed From this Analysis:}

\section{5.}

Actual by Predicted Plot

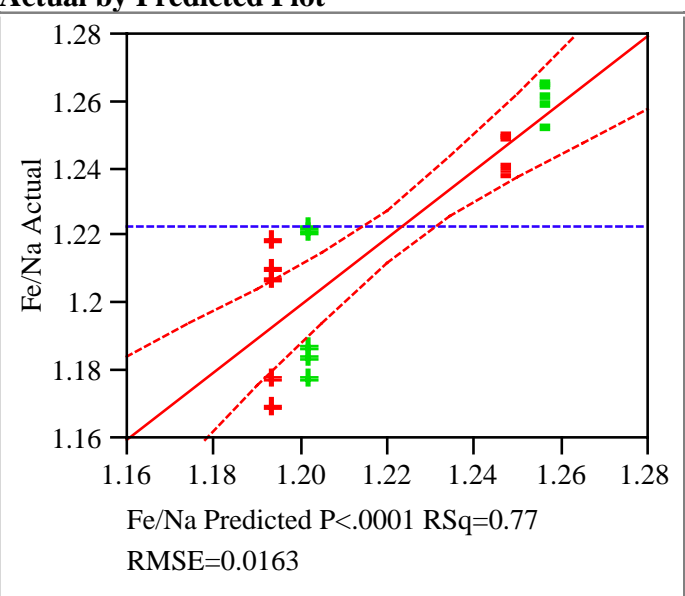

\section{Summary of Fit}

RSquare

RSquare Adj

0.774929

Root Mean Squar

0.746795

0.016289

Mean of Response

$$
1.223317
$$

Observations (or Sum Wgts)

Analysis of Variance

Source DF Sum of Squares

$\begin{array}{lll}\text { Model } & 2 & 0.01461588\end{array}$

$\begin{array}{lll}\text { Error } & 16 & 0.00424505\end{array}$

$\begin{array}{lll}\text { C. Total } & 18 & 0.01886093\end{array}$

Lack Of Fit

$\begin{array}{lrr}\text { Source } & \text { DF } & \text { Sum of Squares } \\ \text { Lack Of Fit } & 1 & 0.00023971 \\ \text { Pure Error } & 15 & 0.00400534 \\ \text { Total Error } & 16 & 0.00424505\end{array}$

19

$\begin{array}{rr}\text { Mean Square } & \text { F Ratio } \\ 0.007308 & 27.5444\end{array}$ $\begin{array}{rr}0.007308 & 27.5444 \\ 0.000265 & \text { Prob > F }\end{array}$

0.000265 Prob $>$ F
$<.0001$

Mean Square

$$
0.000240
$$

0.000267

F Ratio

0.8977

Prob > F

0.3584

Max RSq

0.7876

Parameter Estimates

Term

Intercept

Sample Type[32]

Calibration[1]

Effect Tests

Source

Sample Type

Calibration

Nons

Estimate

$$
\begin{aligned}
& 1.2245226 \\
& -0.004297 \\
& 0.0272018
\end{aligned}
$$
0.003748 0.003748 0.003748

t Ratio 326.73

$$
-1.15
$$

$$
7.26
$$

\section{Prob $>|t|$}

\begin{tabular}{|c|c|c|c|}
\hline \multicolumn{4}{|c|}{ Least Squares Means Table } \\
\hline Level & Least Sq Mean & Std Error & Mean \\
\hline 32 & 1.2202254 & 0.00544545 & 1.21720 \\
\hline 60 & 1.2288198 & 0.00515088 & 1.22882 \\
\hline
\end{tabular}

$<.0001$ 0.2684 $<.0001$

$\begin{array}{rrrrr}\text { Nparm } & \text { DF } & \text { Sum of Squares } & \text { F Ratio } & \text { Prob > F } \\ 1 & 1 & 0.00034881 & 1.3147 & 0.2684 \\ 1 & 1 & 0.01397664 & 52.6793 & <.0001\end{array}$

Residual by Predicted Plot

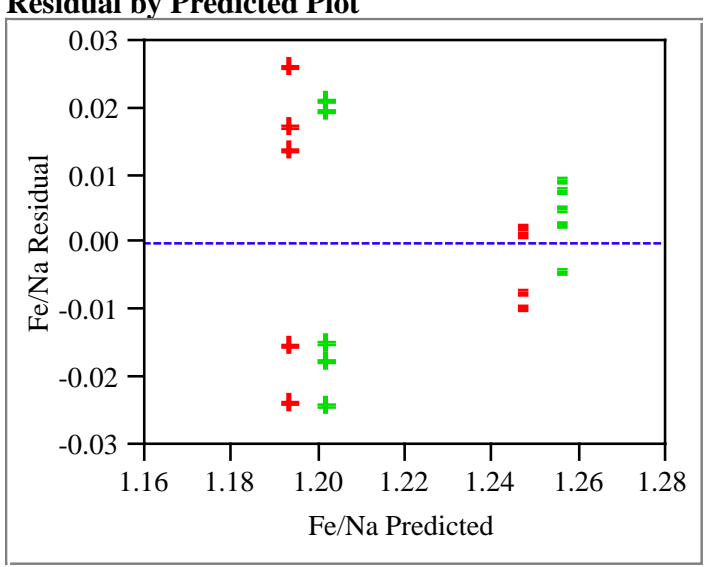

Power Details

$\begin{array}{rrrrr}\text { Alpha } & \text { Sigma } & \text { Delta } & \text { Number } & \text { Power } \\ 0.0500 & 0.016289 & 0.030721 & 19 & 1.0000\end{array}$


Appendix (continued)

Exhibit A6. Plots of the Selected Fission Product and Actinide Concentrations (as micrograms per gram of slurry) by Mass Number Versus Type of Composite and ICP Calibration Block

Oneway Analysis of Tc-99 By Sample/ Calibration
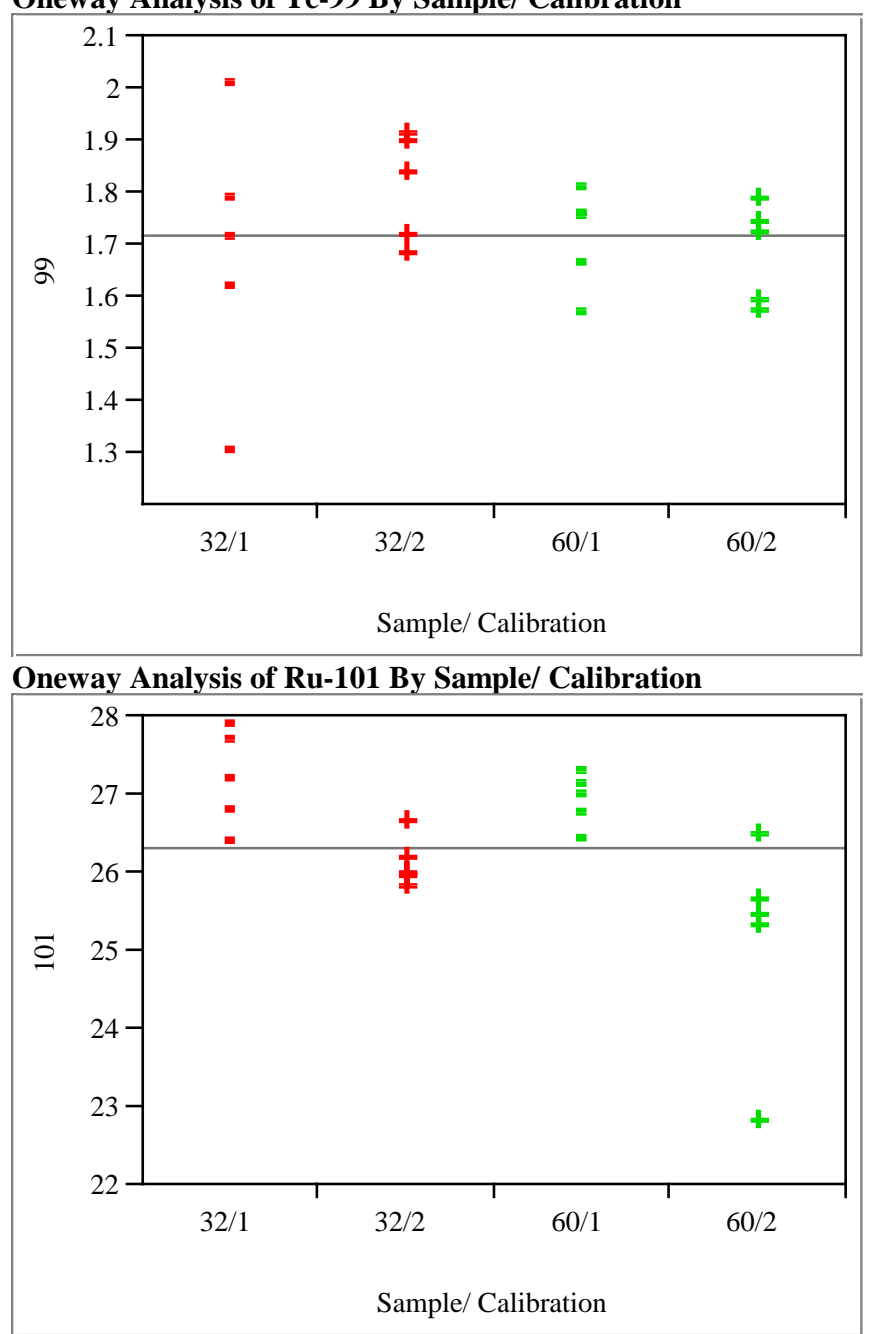

Oneway Analysis of Ru-102 By Sample/ Calibration

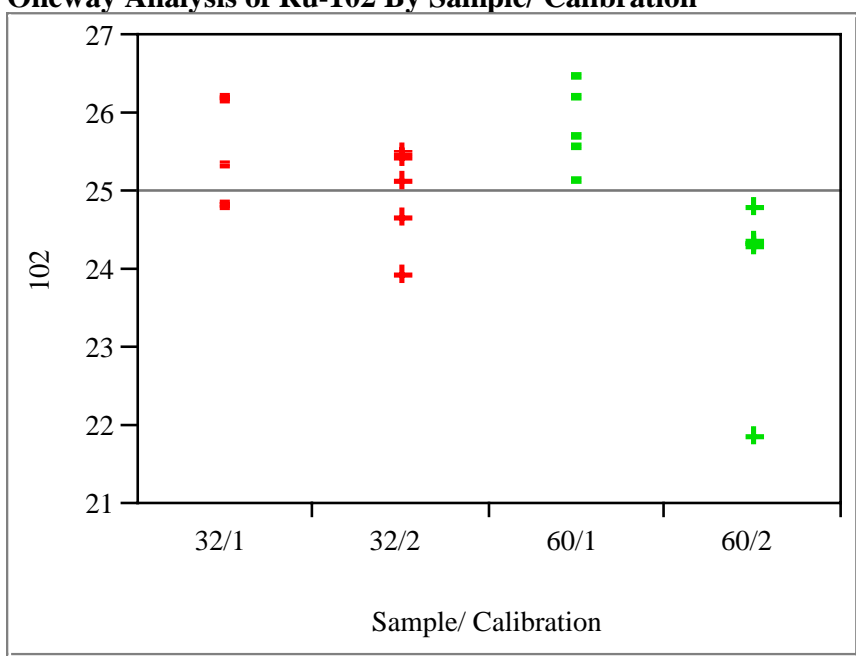

Oneway Analysis of Rh-103 By Sample/ Calibration

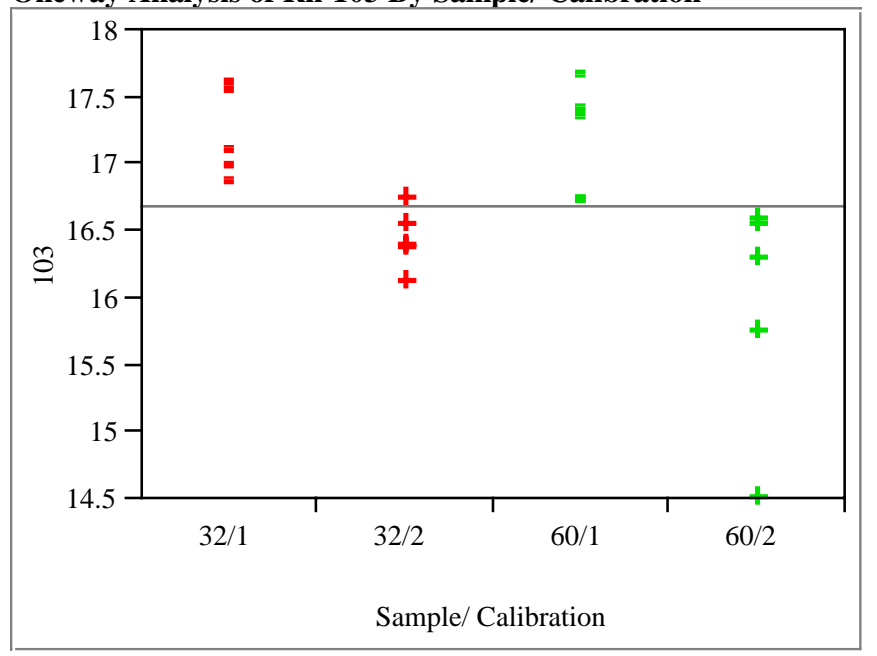




\section{Appendix (continued)}

Exhibit A6. Plots of the Selected Fission Product and Actinide Concentrations (as micrograms per gram of slurry) by Mass Number Versus Type of Composite and ICP Calibration Block (continued)

Oneway Analysis of Ru-104 By Sample/ Calibration

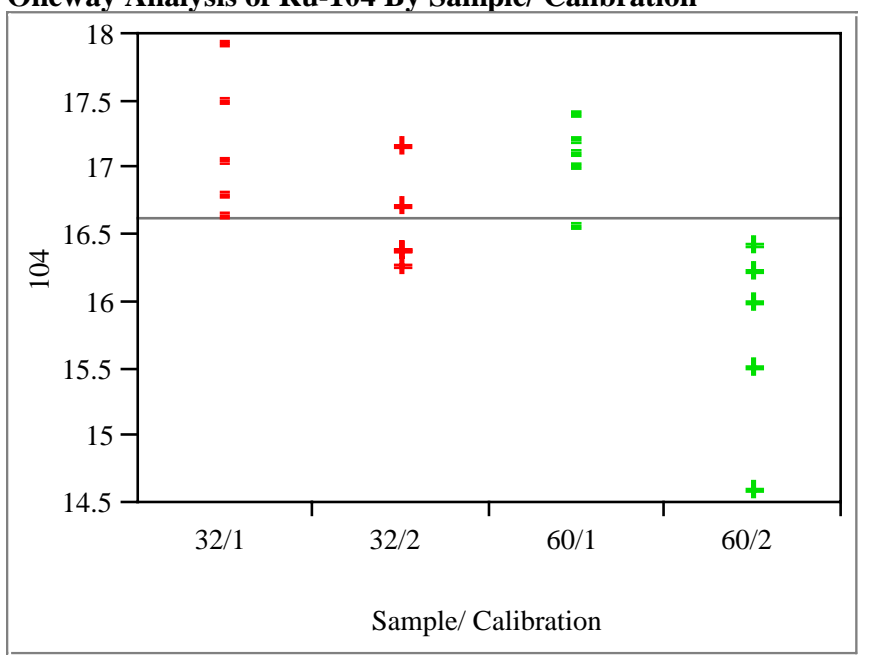

Oneway Analysis of Pd-105 By Sample/ Calibration

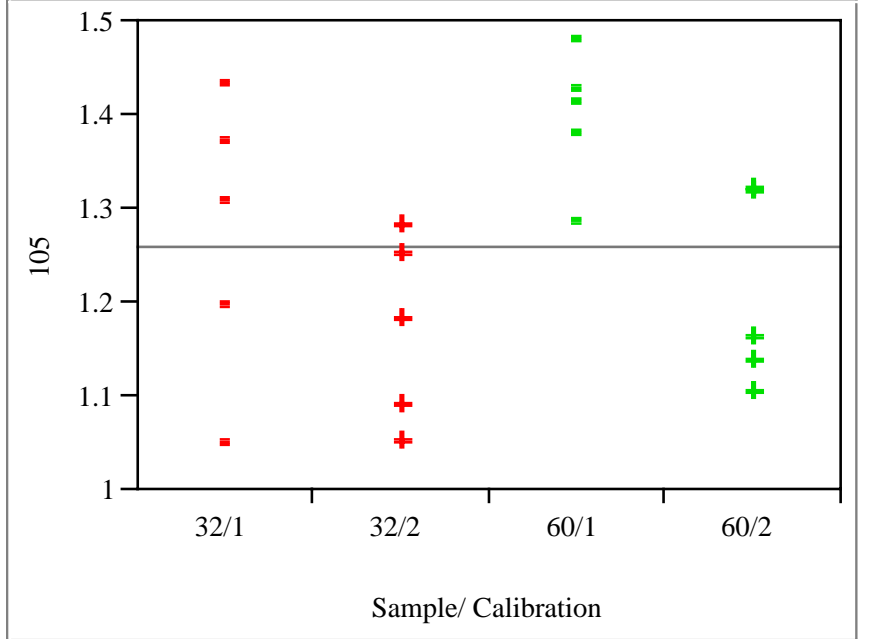

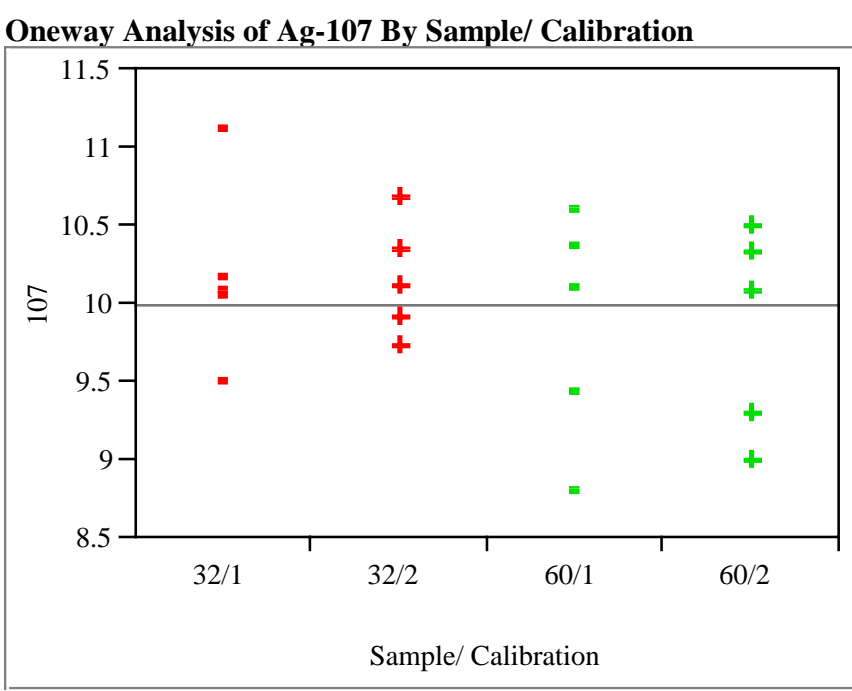

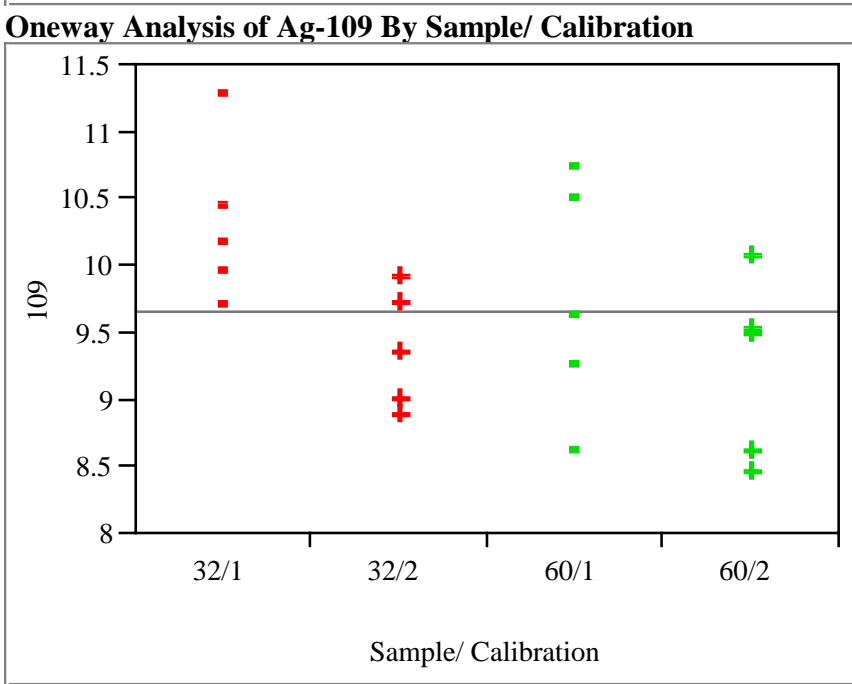




\section{Appendix (continued)}

Exhibit A6. Plots of the Selected Fission Product and Actinide Concentrations (as micrograms per gram of slurry) by Mass Number Versus Type of Composite and ICP Calibration Block (continued)
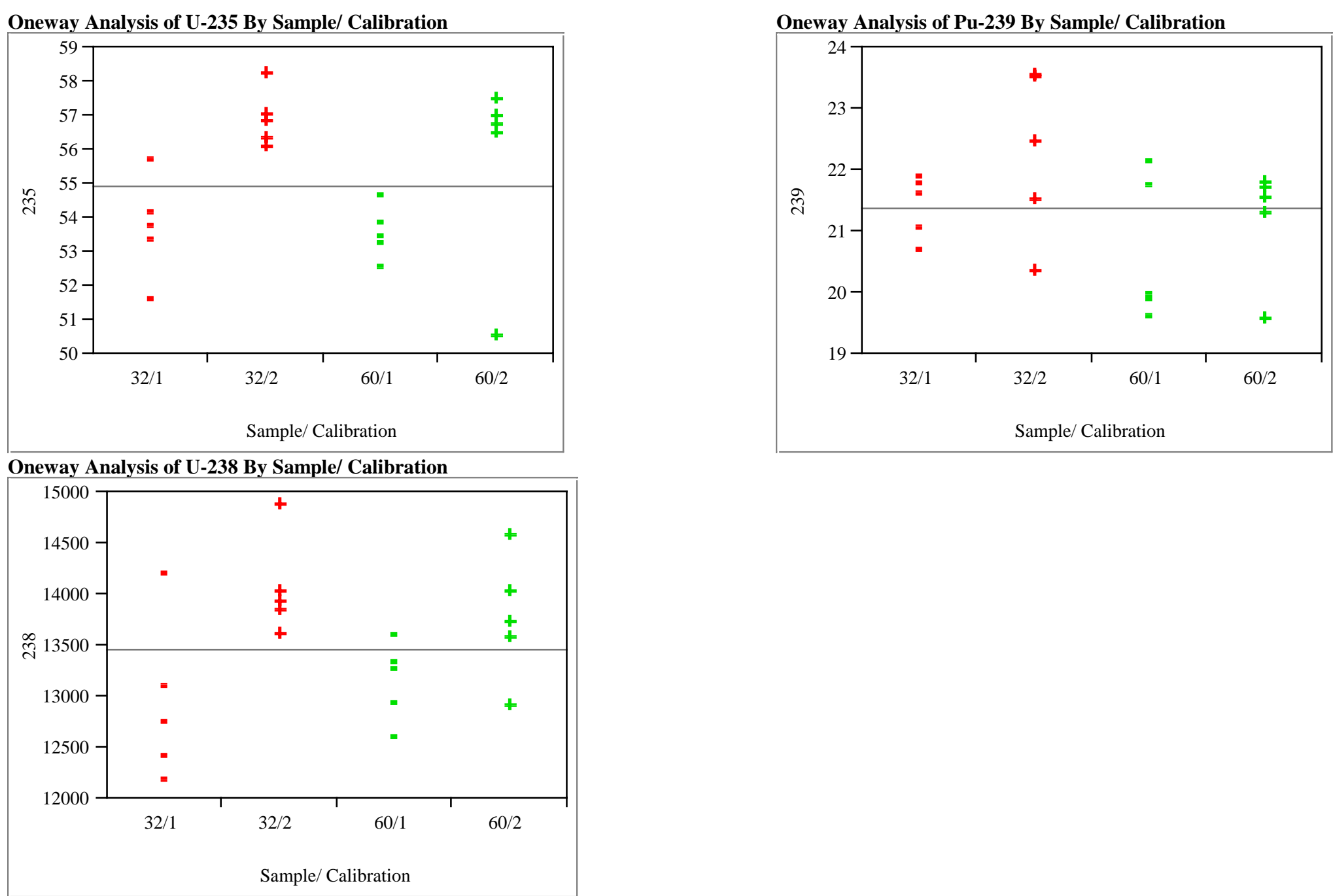


\section{Appendix (continued)}

\section{Exhibit A7. Statistical Analyses of Selected Fission Product and Actinide Concentrations as Micrograms per Gram of Slurry by Type of Composite and ICP Calibration Block}

\section{Response Tc-99}

Whole Model

Actual by Predicted Plot

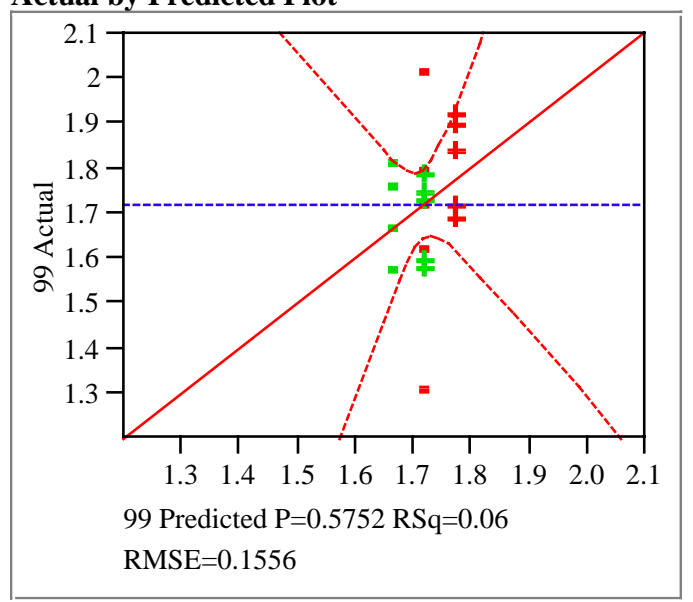

Residual by Predicted Plot

\section{Summary of Fit}

R-square is the portion of variation attributed to the model, between 0 and 1. Root Mean Squared Error "RMSE" estimates the standard deviation of the residual.
RSquare
0.062987
RSquare Adj
$-0.04725$
Root Mean Square Erro
0.155614
Mean of Response
1.719386
Observations (or Sum Wgts)
20

Analysis of Variance

The test that the whole model fits better than a simple mean, i.e. testing that all the parameters are zero except the intercept

Source DF Sum of Squares Mean Square F Ratio

$\begin{array}{lllll}\text { Model } & 2 & 0.02767285 & 0.013836 & 0.5714\end{array}$

$\begin{array}{lllll}\text { Error } & 17 & 0.41166808 & 0.024216 & \text { Prob > F }\end{array}$

$\begin{array}{llll}\text { C. Total } & 19 & 0.43934093 & 0.5752\end{array}$

\section{Lack Of Fit}

Using replicated points as the part of residual error that does not depend on the form of the model so that you can test for the adequacy of the form of the model.

Source

Lack Of Fit $\quad$ D

0.02777639

Pure Error $\quad 16 \quad 0.38389169$

Total Error $\quad 17 \quad 0.41166808$

$\begin{array}{rr}\text { Mean Square } & \text { F Ratio } \\ 0.027776 & 1.1577 \\ 0.023993 & \text { Prob > F } \\ & 0.2979 \\ & \text { Max RSq } \\ & 0.1262\end{array}$

Parameter Estimates

Term

Estimate 1.7193856

0.0254909

$-0.02709$

Composite Type[32]

Calibration[1]

Effect Tests

Source

Composite Type

$\begin{array}{llllll}\text { Composite Type } & 1 & 1 & 0.01299577 & 0.5367 & 0.4738 \\ \text { Calibration } & 1 & 1 & 0.01467708 & 0.6061 & 0.4470\end{array}$
Std Error 0.034796 0.034796 0.034796

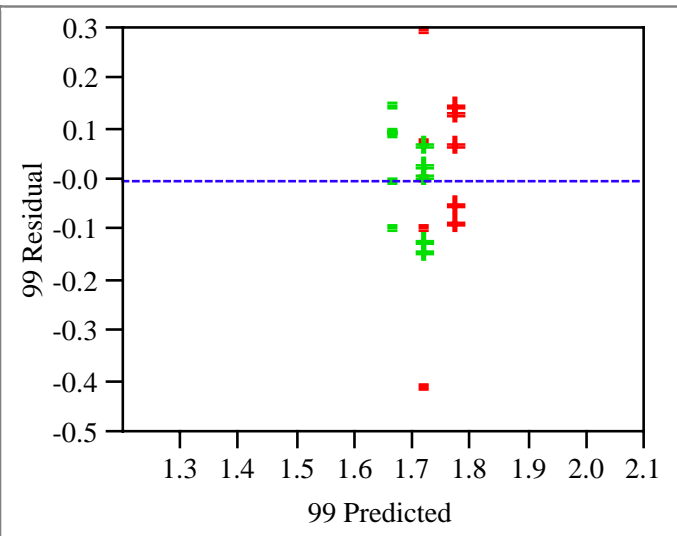


WSRC-TR-2001-00565

Revision 0

Page 48 of 83

November 26, 2001

\section{Appendix (continued)}

\section{Exhibit A7. Statistical Analyses of Selected Fission Product and Actinide Concentrations as Micrograms per Gram of Slurry by Type of Composite and ICP Calibration Block \\ (continued)}

\section{Response Ru-101}

Whole Model

Actual by Predicted Plot

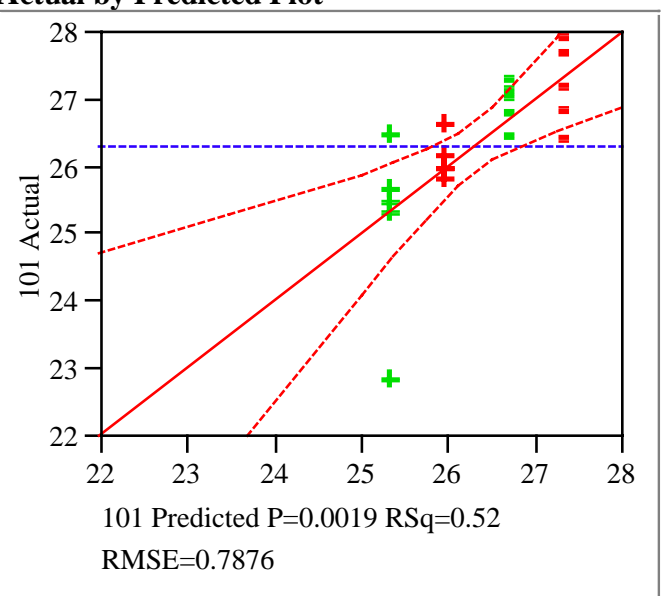

Residual by Predicted Plot

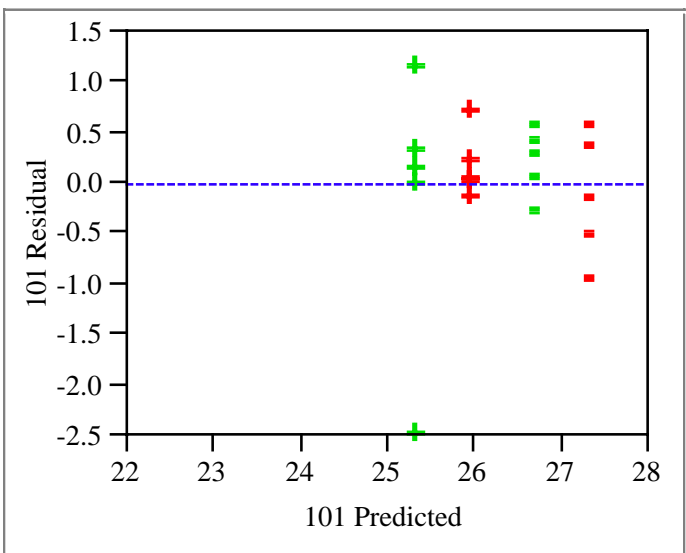

\section{Summary of Fit}

R-square is the portion of variation attributed to the model, between 0 and 1. Root Mean Squared Error "RMSE" estimates the standard deviation of the residual.
RSquare
0.521988
RSquare Adj
0.465752
Root Mean Square Error
0.787553
Mean of Response
26.322
Observations (or Sum Wgts)
20

Analysis of Variance

The test that the whole model fits better than a simple mean, i.e. testing that all the parameters are zero except the intercept

Source DF Sum of Squares Mean Square F Ratio

$\begin{array}{lllll}\text { Model } & 2 & 11.514126 & 5.75706 & 9.2820\end{array}$

$\begin{array}{llll}\text { Error } & 17 & 10.544078 & 0.62024 \text { Prob > F }\end{array}$

$\begin{array}{llll}\text { C. Total } & 19 & 22.058204 & 0.0019\end{array}$

\section{Lack Of Fit}

Using replicated points as the part of residual error that does not depend on the form of the model so that you can test for the adequacy of the form of the model.

Source

Lack Of Fit $\quad 1$

Pure Error $\quad 16 \quad 9.940783$

Mean Square F Ratio

$$
\begin{array}{rr}
0.603295 & \text { F Ratio } \\
0.621299
\end{array}
$$

0.621299 Prob > F

Total Error

10.544078

0.3391

Max RSq

0.5493

Parameter Estimates

Term

Intercept

Composite Type[32]

Calibration[1]

Effect Tests

Source

Composite Type

Calibration

Npa

$\begin{array}{rrrr}\text { Estimate } & \text { Std Error } & \text { t Ratio } & \text { Prob>|t| } \\ 26.322003 & 0.176102 & 149.47 & <.0001 \\ 0.3067776 & 0.176102 & 1.74 & 0.0996 \\ 0.6939696 & 0.176102 & 3.94 & 0.0011 \\ & & & \\ \text { DF } & \text { Sum of Squares } & \text { F Ratio } & \text { Prob > F } \\ 1 & 1.8822504 & 3.0347 & 0.0996 \\ 1 & 9.6318752 & 15.5293 & 0.0011\end{array}$


WSRC-TR-2001-00565

Revision 0

Page 49 of 83

November 26, 2001

\section{Appendix (continued)}

\section{Exhibit A7. Statistical Analyses of Selected Fission Product and Actinide Concentrations as Micrograms per Gram of Slurry by Type of Composite and ICP Calibration Block \\ (continued)}

Response Ru-102

Whole Model

Actual by Predicted Plot

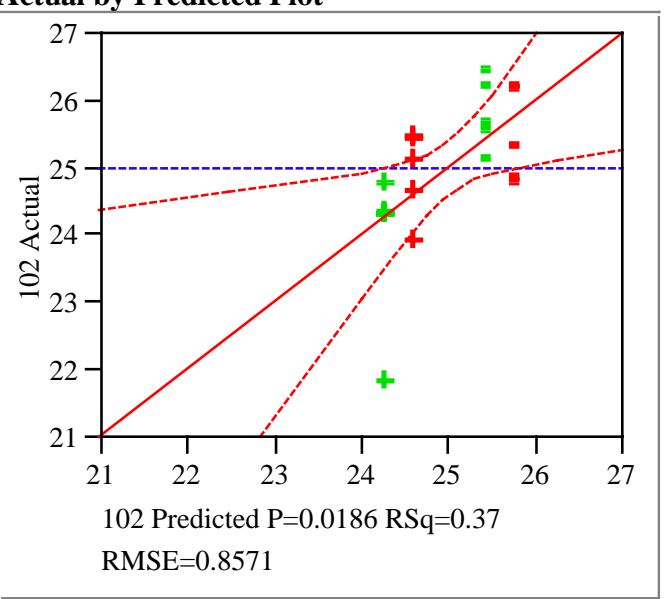

Residual by Predicted Plot

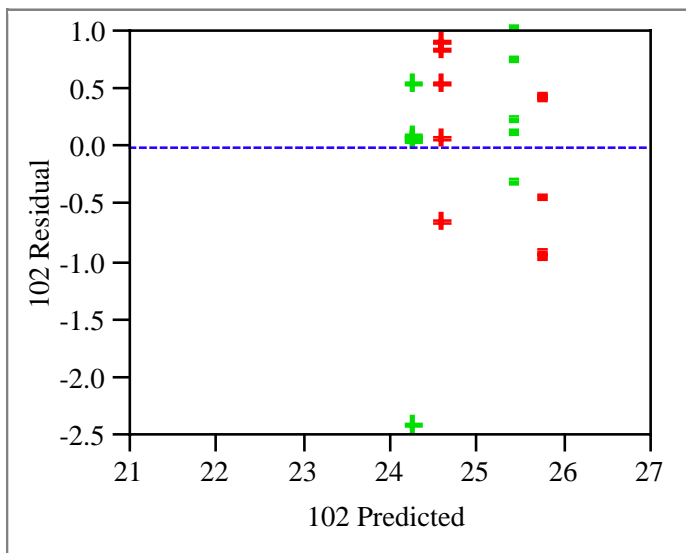

\section{Summary of Fit}

R-square is the portion of variation attributed to the model, between 0 and 1. Root Mean Squared Error "RMSE" estimates the standard deviation of the residual.
RSquare
0.374058
RSquare Adj
0.300417
Root Mean Square Error $\quad 0.857065$
Mean of Response $\quad 25.00461$
Observations (or Sum Wgts)
20

Analysis of Variance

The test that the whole model fits better than a simple mean, i.e. testing that all the parameters are zero except the intercept

Source DF Sum of Squares Mean Square F Ratio

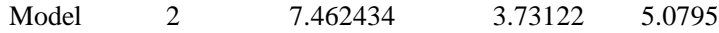

$\begin{array}{lllll}\text { Error } & 17 & 12.487529 & 0.73456 \quad \text { Prob }>\text { F }\end{array}$

$\begin{array}{llll}\text { C. Total } & 19 & 19.949963 & 0.0186\end{array}$

Lack Of Fit

Using replicated points as the part of residual error that does not depend on the form of the model so that you can test for the adequacy of the form of the model.

Source

Lack Of Fit $\quad$ DF

Pure Error $\quad 16$

Sum of Squares

2.217246

Mean Square

2.21725
0.64189

F Ratio

3.4542

Total Error

10.270283

0.64189 Prob > F

0.0816

Max RSq

0.4852

Parameter Estimates

Term

Intercept

Composite Type[32]

Calibration[1]

Effect Tests

Source

Composite Type

Calibration

Nparm

Estimate Std Error t Ratio Prob $>|\mathbf{t}|$

$\begin{array}{lrrr}25.004611 & 0.191646 & 130.47 & <.0001\end{array}$

$\begin{array}{llll}0.1649316 & 0.191646 & 0.86 & 0.4014\end{array}$

$\begin{array}{rrrr}0.588149 & 0.191646 & 3.07 & 0.0070\end{array}$

Dparm Sum of Squares F Ratio Prob > F

$\begin{array}{lrrr}1 & 0.5440485 & 0.7406 & 0.4014 \\ 1 & 6.9183859 & 9.4184 & 0.0070\end{array}$


WSRC-TR-2001-00565

Revision 0

Page 50 of 83

November 26, 2001

\section{Appendix (continued)}

\section{Exhibit A7. Statistical Analyses of Selected Fission Product and Actinide Concentrations as Micrograms per Gram of Slurry by Type of Composite and ICP Calibration Block \\ (continued)}

Response Rh-103

Whole Model

Actual by Predicted Plot

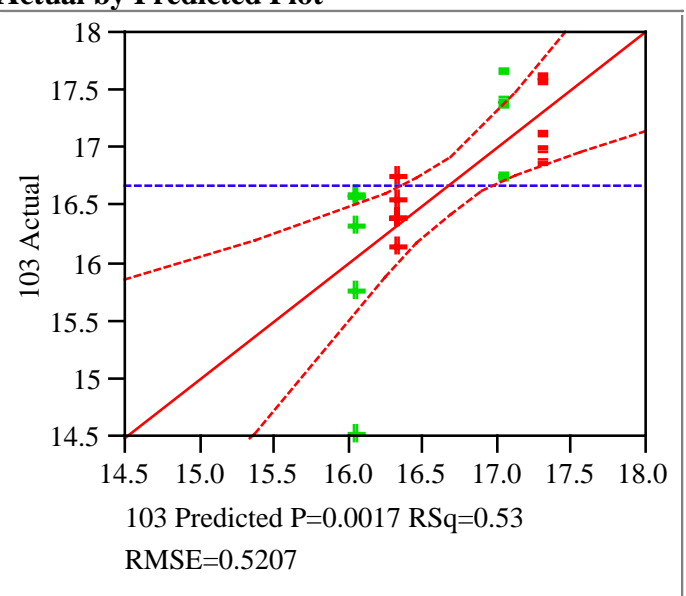

Residual by Predicted Plot

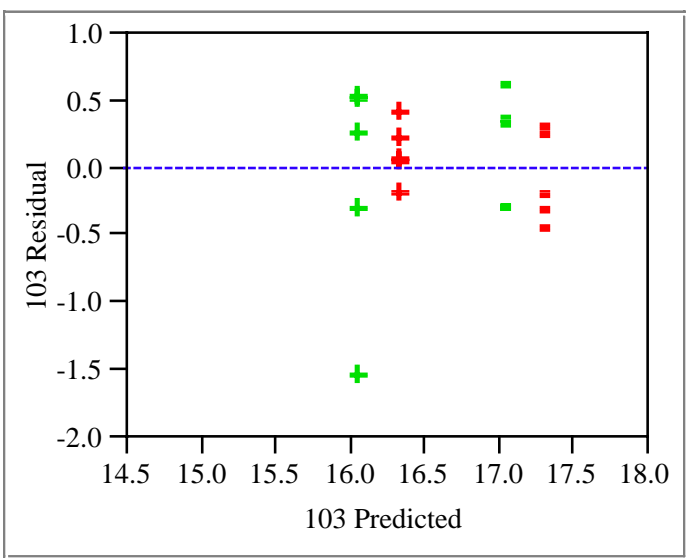

Summary of Fit

R-square is the portion of variation attributed to the model, between 0 and 1. Root Mean Squared Error "RMSE" estimates the standard deviation of the residual.

RSquare 0.529348

RSquare Adj $\quad 0.473977$

Root Mean Square Error $\quad 0.520707$

Mean of Response $\quad 16.68221$

Observations (or Sum Wgts) 20

Analysis of Variance

The test that the whole model fits better than a simple mean, i.e. testing that all the parameters are zero except the intercept

Source DF Sum of Squares Mean Square F Ratio

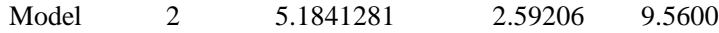

$\begin{array}{lllll}\text { Error } & 17 & 4.6093022 & 0.27114 & \text { Prob }>\text { F }\end{array}$

$\begin{array}{llll}\text { C. Total } & 19 & 9.7934303 & 0.0017\end{array}$

Lack Of Fit

Using replicated points as the part of residual error that does not depend on the form of the model so that you can test for the adequacy of the form of the model.

Source

Lack Of Fit $\quad$ DF

0.2540878

Pure Error $\quad 16 \quad 4.3552144$

Mean Square F Ratio $0.254088 \quad 0.9335$

Total Error $\quad 17 \quad 4.6093022$

$0.272201 \quad$ Prob > F

0.3483

Max RSq

0.5553

Parameter Estimates

Term

Intercept

Composite Type[32]

Calibration[1]

Effect Tests

Source

Composite Type

Calibration

Npar




WSRC-TR-2001-00565

Revision 0

Page 51 of 83

November 26, 2001

\section{Appendix (continued)}

\section{Exhibit A7. Statistical Analyses of Selected Fission Product and Actinide Concentrations as Micrograms per Gram of Slurry by Type of Composite and ICP Calibration Block

\author{
(continued)
}

Response Ru-104

Whole Model

Actual by Predicted Plot

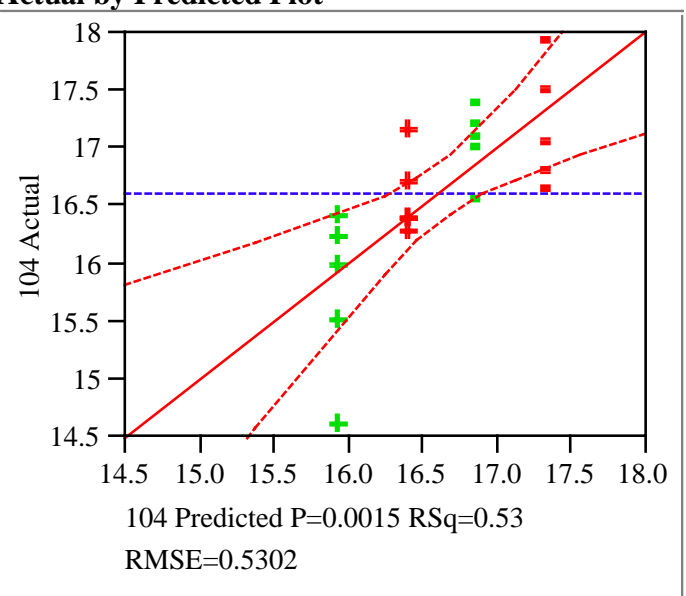

Residual by Predicted Plot

Summary of Fit

$\mathrm{R}$-square is the portion of variation attributed to the model, between 0 and 1. Root Mean Squared Error "RMSE" estimates the standard deviation of the residual.

RSquare

0.534005

RSquare Adj

0.479182

0.5302

Root Mean Square Erro

16.62251

Mean of Response

20

Analysis of Variance

The test that the whole model fits better than a simple mean, i.e. testing that all the parameters are zero except the intercept

Source DF Sum of Squares Mean Square F Ratio

$\begin{array}{lllll}\text { Model } & 2 & 5.476375 & 2.73819 & 9.7405\end{array}$

$\begin{array}{lllll}\text { Error } & 17 & 4.778911 & 0.28111 & \text { Prob }>\text { F }\end{array}$

$\begin{array}{llll}\text { C. Total } & 19 & 10.255286 & 0.0015\end{array}$

Lack Of Fit

Using replicated points as the part of residual error that does not depend on the form of the model so that you can test for the adequacy of the form of the model.

Source

$\begin{array}{rr}\text { DF } & \text { Sum of Squares } \\ 1 & 0.6331798\end{array}$

Mean Square 0.633180

F Ratio

Lack Of Fit

0.259108

2.4437

Prob > F

0.1376

Total Error

4.7789113

Max RSq

Parameter Estimates

Term

Intercept

Composite Type[32]

Calibration[1]

Effect Tests

Source

Composite Type

Calibration

$\begin{array}{rrrrr}\text { Nparm } & \text { DF } & \text { Sum of Squares } & \text { F Ratio } & \text { Prob }>\text { F } \\ 1 & 1 & 1.1505172 & 4.0927 & 0.0591 \\ 1 & 1 & 4.3258578 & 15.3884 & 0.0011\end{array}$


WSRC-TR-2001-00565

Revision 0

Page 52 of 83

November 26, 2001

\section{Appendix (continued)}

\section{Exhibit A7. Statistical Analyses of Selected Fission Product and Actinide Concentrations as Micrograms per Gram of Slurry by Type of Composite and ICP Calibration Block

\author{
(continued)
}

Response Pd-105

Whole Model

Actual by Predicted Plot



Residual by Predicted Plot

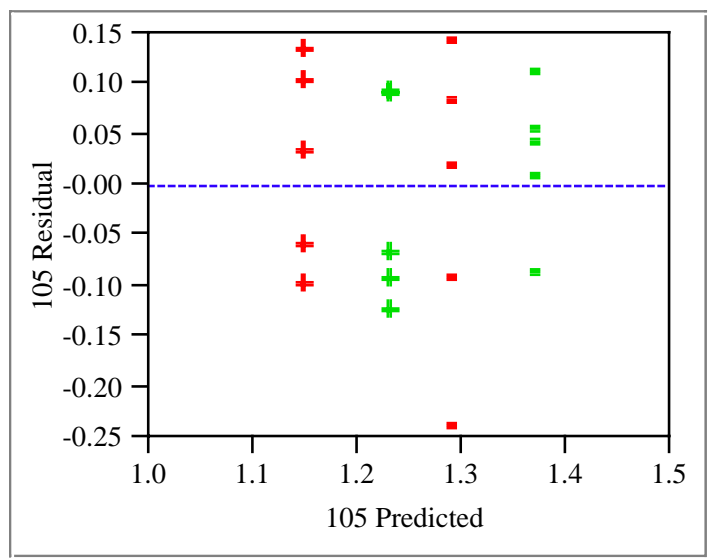

Summary of Fit

R-square is the portion of variation attributed to the model, between 0 and 1. Root Mean Squared Error "RMSE" estimates the standard deviation of the residual.

RSquare $\quad 0.391163$

RSquare Adj $\quad 0.319535$

Root Mean Square Error $\quad 0.10999$

Mean of Response 1.260321

Observations (or Sum Wgts) $\quad 20$

Analysis of Variance

The test that the whole model fits better than a simple mean, i.e. testing that all the parameters are zero except the intercept

Source DF Sum of Squares Mean Square F Ratio

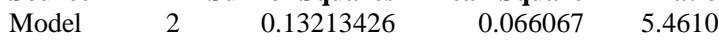

$\begin{array}{lllll}\text { Error } & 17 & 0.20566438 & 0.012098 \quad \text { Prob > F }\end{array}$

$\begin{array}{llll}\text { C. Total } & 19 & 0.33779864 & 0.0147\end{array}$

Lack Of Fit

Using replicated points as the part of residual error that does not depend on the form of the model so that you can test for the adequacy of the form of the model.

$\begin{array}{lrrrr}\text { Source } & \text { DF } & \text { Sum of Squares } & \text { Mean Square } & \text { F Ratio } \\ \text { Lack Of Fit } & 1 & 0.00952905 & 0.009529 & 0.7773 \\ \text { Pure Error } & 16 & 0.19613533 & 0.012258 & \text { Prob > F } \\ \text { Total Error } & 17 & 0.20566438 & & 0.3910 \\ & & & & \text { Max RSq } \\ & & & & 0.4194\end{array}$

Parameter Estimates

Term

Intercept

Composite Type[32]

Calibration[1]

Effect Tests

Source

Composite Type

Calibration

$\begin{array}{rrrr}\text { Estimate } & \text { Std Error } & \text { t Ratio } & \text { Prob }>|\mathbf{t}| \\ 1.2603207 & 0.024595 & 51.24 & <.0001 \\ -0.04055 & 0.024595 & -1.65 & 0.1176 \\ 0.0704446 & 0.024595 & 2.86 & 0.0107\end{array}$

$\begin{array}{rrrrr}\text { Nparm } & \text { DF } & \text { Sum of Squares } & \text { F Ratio } & \text { Prob > F } \\ 1 & 1 & 0.03288556 & 2.7183 & 0.1176 \\ 1 & 1 & 0.09924870 & 8.2038 & 0.0107\end{array}$


WSRC-TR-2001-00565

Revision 0

Page 53 of 83

November 26, 2001

\section{Appendix (continued)}

\section{Exhibit A7. Statistical Analyses of Selected Fission Product and Actinide Concentrations as Micrograms per Gram of Slurry by Type of Composite and ICP Calibration Block

\author{
(continued)
}

Response Ag-107

Whole Model

Actual by Predicted Plot

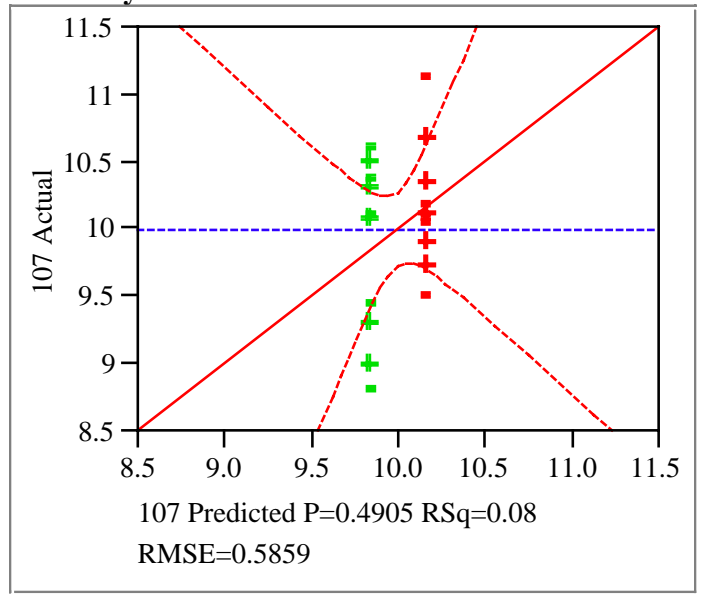

Residual by Predicted Plot

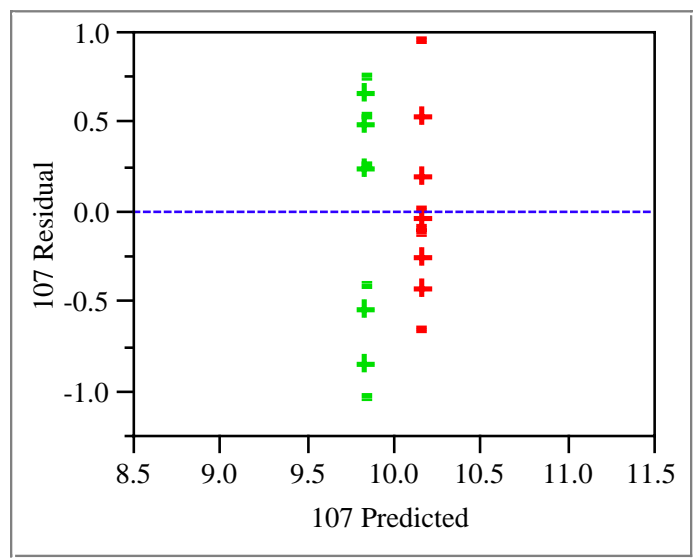

Summary of Fit

R-square is the portion of variation attributed to the model, between 0 and 1. Root Mean Squared Error "RMSE" estimates the standard deviation of the residual.

RSquare

RSquare Adj

0.080398

Mean of Response

Observations (or Sum Wgts) 20

Analysis of Variance

The test that the whole model fits better than a simple mean, i.e. testing that all the parameters are zero except the intercept

Source DF Sum of Squares Mean Square F Ratio

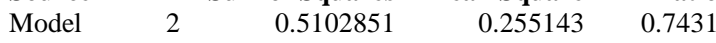

$\begin{array}{lllll}\text { Error } & 17 & 5.8367019 & 0.343335 & \text { Prob > F }\end{array}$

$\begin{array}{lllr}\text { C. Total } & 19 & 6.3469869 & 0.4905\end{array}$

Lack Of Fit

Using replicated points as the part of residual error that does not depend on the form of the model so that you can test for the adequacy of the form of the model.

$\begin{array}{lrrrr}\text { Source } & \text { DF } & \text { Sum of Squares } & \text { Mean Square } & \text { F Ratio } \\ \text { Lack Of Fit } & 1 & 0.0000003 & 0.000000 & 0.0000 \\ \text { Pure Error } & 16 & 5.8367016 & 0.364794 & \text { Prob > F } \\ \text { Total Error } & 17 & 5.8367019 & & 0.9993 \\ & & & & \text { Max RSq } \\ & & & & 0.0804\end{array}$

Parameter Estimates

Term

Estimate

Std Error

t Ratio Prob $>|t|$

Intercept

9.996063

0.131022

76.29

0.1597058

0.131022

1.22

0.02

$<.0001$

Calibration[1]

Effect Tests

Source

Composite Type

Nparm DF

0.131022

0.9827

Calibration

$\begin{array}{rrrrr}1 & 1 & 0.51011871 & 1.4858 & 0.2395 \\ 1 & 1 & 0.00016634 & 0.0005 & 0.9827\end{array}$

F Ratio Prob > F

$\begin{array}{llllll} & 1 & 1 & 0.00016634 & 0.0005 & 0.9827\end{array}$


WSRC-TR-2001-00565

Revision 0

Page 54 of 83

November 26, 2001

Appendix (continued)

Exhibit A7. Statistical Analyses of Selected Fission Product and Actinide Concentrations as Micrograms per Gram of Slurry by Type of Composite and ICP Calibration Block

(continued)

Response Ag-109

Whole Model

Actual by Predicted Plot

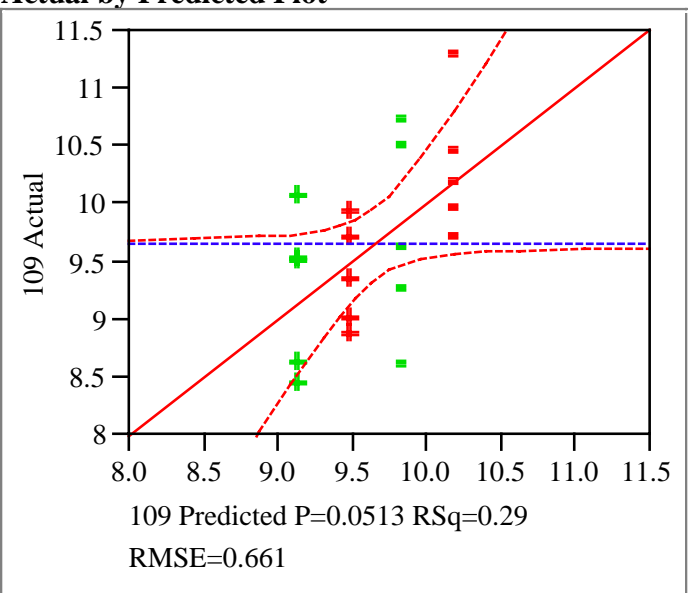

Residual by Predicted Plot



Summary of Fit

R-square is the portion of variation attributed to the model, between 0 and 1. Root Mean Squared Error "RMSE" estimates the standard deviation of the residual.

RSquare 0.294982

RSquare Adj

0.212039

Root Mean Square Error $\quad 0.661038$

Mean of Response $\quad 9.653543$

Observations (or Sum Wgts) 20

Analysis of Variance

The test that the whole model fits better than a simple mean, i.e. testing that all the parameters are zero except the intercept

Source DF Sum of Squares Mean Square F Ratio



$\begin{array}{lllll}\text { Error } & 17 & 7.428517 & 0.43697 & \text { Prob > F }\end{array}$

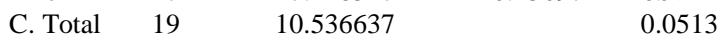

Lack Of Fit

Using replicated points as the part of residual error that does not depend on the form of the model so that you can test for the adequacy of the form of the model.

Source DF

Pure Error $\quad 16 \quad 7.1964448$

Total Error $\quad 17 \quad 7.4285170$

$$
\begin{array}{rr}
\text { Mean Square } & \text { F Ratio } \\
0.232072 & 0.5160 \\
0.449778 & \text { Prob }>\text { F } \\
& 0.4829 \\
& \text { Max RSq } \\
& 0.3170
\end{array}
$$

Parameter Estimates

Term

Estimate Std Error t Ratio Prob $>|\mathbf{t}|$

Intercept

$\begin{array}{llll}9.653543 & 0.147813 & 65.31 & <.0001\end{array}$

Composite Type[32]

Calibration[1]

$\begin{array}{llr}0.1780016 & 0.147813 & 1.20\end{array}$

Effect Tests

Source

Composite Type

$\begin{array}{rrrrr}\text { Nparm } & \text { DF } & \text { Sum of Squares } & \text { F Ratio } & \text { Prob > F } \\ 1 & 1 & 0.6336917 & 1.4502 & 0.2450 \\ 1 & 1 & 2.4744283 & 5.6627 & 0.0293\end{array}$ 
WSRC-TR-2001-00565

Revision 0

Page 55 of 83

November 26, 2001

\section{Appendix (continued)}

\section{Exhibit A7. Statistical Analyses of Selected Fission Product and Actinide Concentrations as Micrograms per Gram of Slurry by Type of Composite and ICP Calibration Block \\ (continued)}

Response U-235

Whole Model

Actual by Predicted Plot

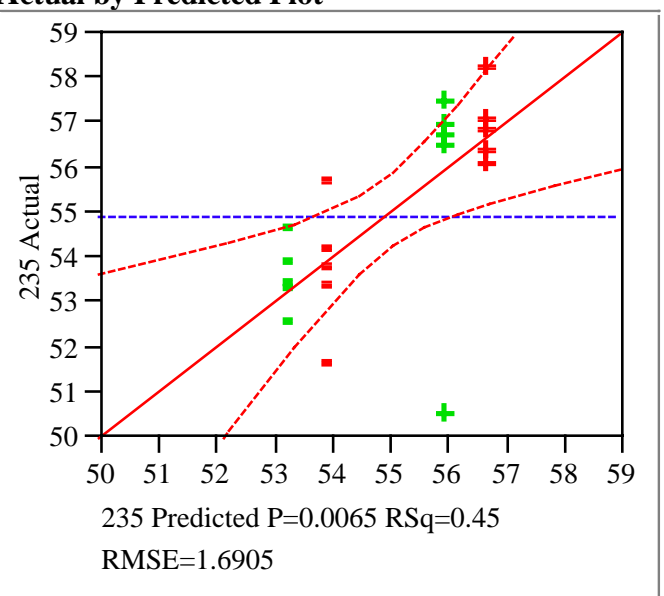

Residual by Predicted Plot



\section{Summary of Fit}

R-square is the portion of variation attributed to the model, between 0 and 1. Root Mean Squared Error "RMSE" estimates the standard deviation of the residual.

RSquare 0.446681

RSquare Adj $\quad 0.381584$

Root Mean Square Error $\quad 1.690543$

Mean of Response $\quad 54.90922$

Observations (or Sum Wgts) 20

Analysis of Variance

The test that the whole model fits better than a simple mean, i.e. testing that all the parameters are zero except the intercept

Source DF Sum of Squares Mean Square F Ratio

$\begin{array}{lllll}\text { Model } & 2 & 39.221359 & 19.6107 & 6.8618\end{array}$

$\begin{array}{llll}\text { Error } & 17 & 48.584898 & 2.8579 \quad \text { Prob }>\text { F }\end{array}$

C. Total $19 \quad 87.806257 \quad 0.0065$

\section{Lack Of Fit}

Using replicated points as the part of residual error that does not depend on the form of the model so that you can test for the adequacy of the form of the model.

Source

Lack Of Fit $\quad$ DF

Pure Error 16

Sum of Squares

1.542866

Mean Square

$\begin{array}{r}1.54287 \\ \hline\end{array}$

F Ratio

0.5248

Total Error

47.042031

2.94013

Prob > F

0.4793

Max RSq

0.4643

Parameter Estimates

Term

Intercept

Composite Type[32]

Calibration[1]

Effect Tests

Source

Composite Type

Calibration

$\begin{array}{rrrrr}\text { Nparm } & \text { DF } & \text { Sum of Squares } & \text { F Ratio } & \text { Prob > F } \\ 1 & 1 & 2.527298 & 0.8843 & 0.3602 \\ 1 & 1 & 36.694061 & 12.8394 & 0.0023\end{array}$


WSRC-TR-2001-00565

Revision 0

Page 56 of 83

November 26, 2001

\section{Appendix (continued)}

\section{Exhibit A7. Statistical Analyses of Selected Fission Product and Actinide Concentrations as Micrograms per Gram of Slurry by Type of Composite and ICP Calibration Block

\author{
(continued)
}

\section{Response U-238}

Whole Model

Actual by Predicted Plot

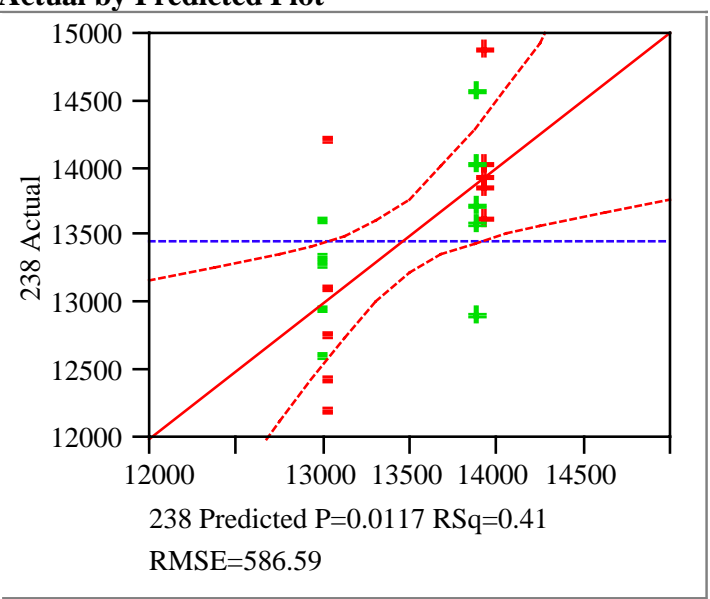

Residual by Predicted Plot

Summary of Fit

$\mathrm{R}$-square is the portion of variation attributed to the model, between 0 and 1. Root Mean Squared Error "RMSE" estimates the standard deviation of the residual.
RSquare
0.407651
RSquare Adj
0.337963
Root Mean Square Erro
586.5918
Mean of Response
13459.74
Observations (or Sum Wgts)
20

Analysis of Variance

The test that the whole model fits better than a simple mean, i.e. testing that all the parameters are zero except the intercept

Source DF Sum of Squares Mean Square F Ratio

$\begin{array}{lllll}\text { Model } & 2 & 4025612.3 & 2012806 & 5.8497\end{array}$

$\begin{array}{lllr}\text { Error } & 17 & 5849529.8 & 344090 \quad \text { Prob }>\text { F }\end{array}$

$\begin{array}{llll}\text { C. Total } & 19 & 9875142.0 & 0.0117\end{array}$

Lack Of Fit

Using replicated points as the part of residual error that does not depend on the form of the model so that you can test for the adequacy of the form of the model.

Source

Lack Of Fit $\quad$ DF

Pure Error $\quad 16$

Sum of Squares

332474.3
5517055.4

Mean Square

332474
344816

F Ratio

Total Error

5849529.8

Prob > F

0.3407

Max RSq

0.4413

Parameter Estimates

Term

Estimate Std Error t Ratio Prob $>|\mathbf{t}|$

Intercept

$\begin{array}{llll}13459.736 & 131.1659 & 102.62 & <.0001\end{array}$

Composite Type[32]

Calibration[1]

Effect Tests

Source

Composite Type

Calibration

$\begin{array}{rrrrr}\text { Nparm } & \text { DF } & \text { Sum of Squares } & \text { F Ratio } & \text { Prob > F } \\ 1 & 1 & 8033.7 & 0.0233 & 0.8804 \\ 1 & 1 & 4017578.6 & 11.6760 & 0.0033\end{array}$

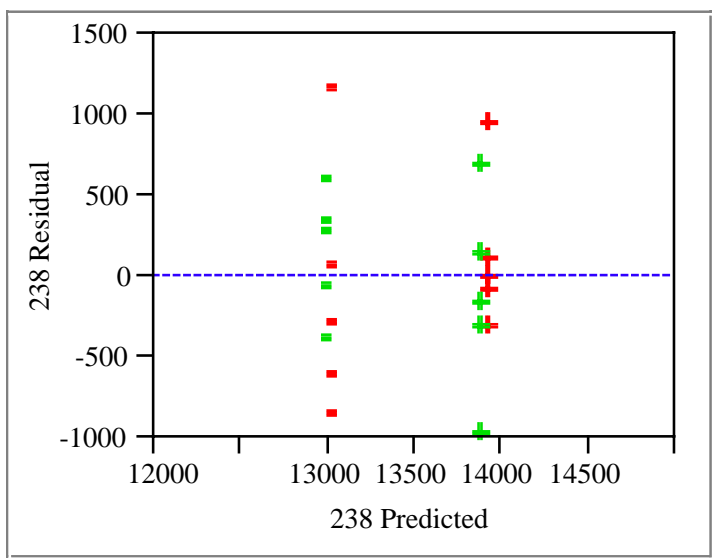


WSRC-TR-2001-00565

Revision 0

Page 57 of 83

November 26, 2001

\section{Appendix (continued)}

\section{Exhibit A7. Statistical Analyses of Selected Fission Product and Actinide Concentrations as Micrograms per Gram of Slurry by Type of Composite and ICP Calibration Block

\author{
(continued)
}

Response Pu-239

Whole Model

Actual by Predicted Plot

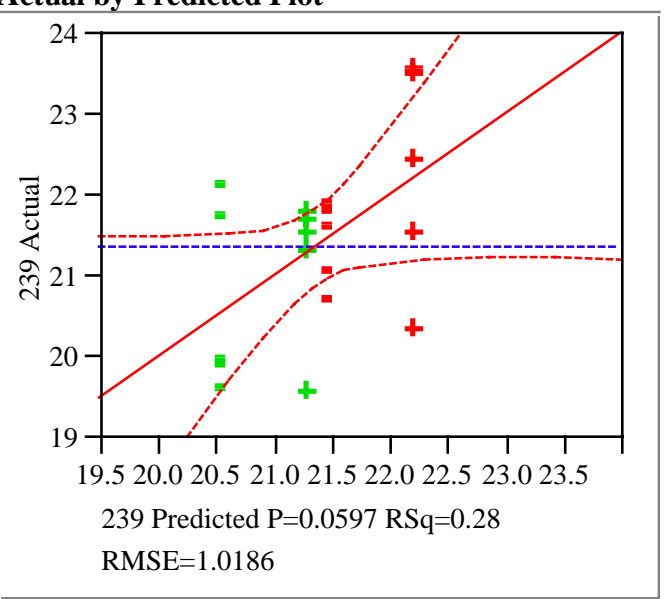

Residual by Predicted Plot

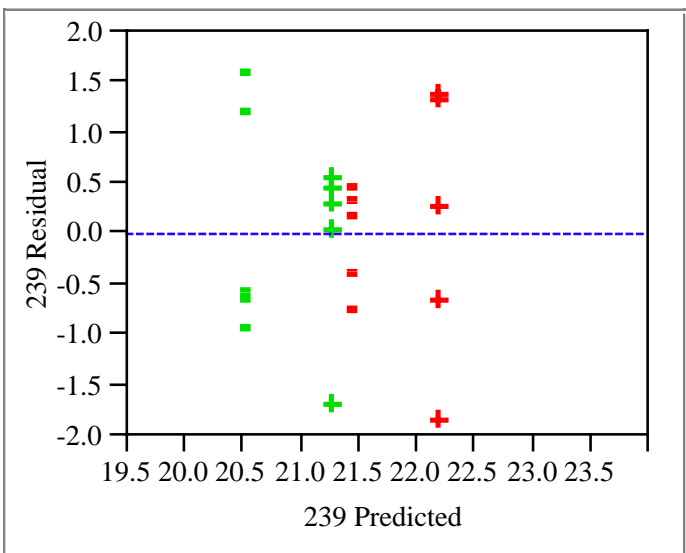

Summary of Fit

R-square is the portion of variation attributed to the model, between 0 and 1. Root Mean Squared Error "RMSE" estimates the standard deviation of the residual.

RSquare 0.282155

RSquare Adj $\quad 0.197702$

Root Mean Square Error $\quad 1.018567$

Mean of Response 21.36172

Observations (or Sum Wgts) 20

Analysis of Variance

The test that the whole model fits better than a simple mean, i.e. testing that all the parameters are zero except the intercept

Source DF Sum of Squares Mean Square F Ratio

$\begin{array}{lllll}\text { Model } & 2 & 6.932416 & 3.46621 & 3.3410\end{array}$

$\begin{array}{lllll}\text { Error } & 17 & 17.637154 & 1.03748 & \text { Prob }>\text { F }\end{array}$

$\begin{array}{llll}\text { C. Total } & 19 & 24.569571 & 0.0597\end{array}$

Lack Of Fit

Using replicated points as the part of residual error that does not depend on the form of the model so that you can test for the adequacy of the form of the model.

Source

F Sum of Squares

Lack Of Fit 1

Pure Error 16

0.147923

17.489231

Mean Square

0.14792
1.09308

F Ratio

0.1353

Total Error

17.637154

1.09308

Prob > F

0.7178

Max RSq

0.2882

Parameter Estimates

Term

Intercept

Composite Type[32]

Calibration[1]

Effect Tests

Source

Composite Type

Calibration

$\begin{array}{rrrr}\text { Estimate } & \text { Std Error } & \text { t Ratio } & \text { Prob }>|\mathbf{t}| \\ 21.361715 & 0.227759 & 93.79 & <.0001 \\ 0.4597447 & 0.227759 & 2.02 & 0.0596 \\ -0.367771 & 0.227759 & -1.61 & 0.1248\end{array}$

$\begin{array}{rrrrr}\text { Nparm } & \text { DF } & \text { Sum of Squares } & \text { F Ratio } & \text { Prob > F } \\ 1 & 1 & 4.2273031 & 4.0746 & 0.0596 \\ 1 & 1 & 2.7051132 & 2.6074 & 0.1248\end{array}$


WSRC-TR-2001-00565

Revision 0

Page 58 of 83

November 26, 2001

\section{Appendix (continued)}

\section{Exhibit A8. Sensitivity of Statistical Analyses of the Concentrations (in micrograms per gram of slurry) by Mass Number to $5 \%$ Differences Due to Type of Composite}

\begin{tabular}{|c|c|c|c|c|}
\hline \multicolumn{5}{|c|}{ Response Tc-99 } \\
\hline Level & Least Sq Mea & & Std Error & Mean \\
\hline 32 & 1.744876 & & 0.04920952 & 1.74488 \\
\hline 60 & 1.693894 & & 0.04920952 & 1.69389 \\
\hline \multicolumn{5}{|c|}{ Power Details } \\
\hline Alpha & Sigma & Delta & Number & Power \\
\hline 0.0500 & $0.155614 \quad 0$ & 0.042347 & 20 & 0.2096 \\
\hline
\end{tabular}

Response Ru-101

$\begin{array}{lrrr}\text { Level } & \text { Least Sq Mean } & \text { Std Error } & \text { Mean } \\ 32 & 26.628781 & 0.24904616 & 26.6288 \\ 60 & 26.015226 & 0.24904616 & 26.0152\end{array}$

Power Details

$\begin{array}{rrrrr}\text { Alpha } & \text { Sigma } & \text { Delta } & \text { Number } & \text { Power } \\ 0.0500 & 0.787553 & 0.575381 & 20 & 0.8682\end{array}$

Response Ru-102

Level Least Sq Mean

$32 \quad 25.169542$

$60 \quad 24.839679$

Power Details

Alpha Sigma Delta Number Power

$\begin{array}{lllll}0.0500 & 0.857065 & 620992 & 20 & 0.0500\end{array}$

\section{Response Rh-103}

$\begin{array}{lrrrr}\text { Level } & \text { Least Sq Mean } & \text { Std Error } & \text { Mean } \\ 32 & 16.817161 & 0.16466190 & 16.8172 \\ 60 & 16.547264 & 0.16466190 & 16.5473\end{array}$

Power Details

$\begin{array}{rrrrr}\text { Alpha } & \text { Sigma } & \text { Delta } & \text { Number } & \text { Power } \\ 0.0500 & 0.520707 & 0.004117 & 20 & 0.0501\end{array}$

Response Ru-104

\begin{tabular}{|c|c|c|c|c|c|}
\hline Level & \multicolumn{2}{|c|}{ Least Sq Mean } & \multicolumn{2}{|c|}{ Std Error } & Mean \\
\hline 32 & 16.8 & 62353 & \multicolumn{2}{|c|}{0.16766408} & 16.8624 \\
\hline 60 & 16.3 & 32662 & \multicolumn{2}{|c|}{0.16766408} & 16.3827 \\
\hline \multicolumn{6}{|c|}{ Power Details } \\
\hline & Sigma & Delta & umber & & \\
\hline 0.0500 & 0.5302 & 0.409567 & 20 & & \\
\hline
\end{tabular}

Response Pd-105

$\begin{array}{lrrr}\text { Level } & \text { Least Sq Mean } & \text { Std Error } & \text { Mean } \\ 32 & 1.2197710 & 0.03478204 & 1.21977 \\ 60 & 1.3008704 & 0.03478204 & 1.30087\end{array}$

Power Details

Alpha Sigma Delta Number Power $\begin{array}{lllll}0.0500 & 0.10999 & 0.032522 & 20 & 0.2390\end{array}$

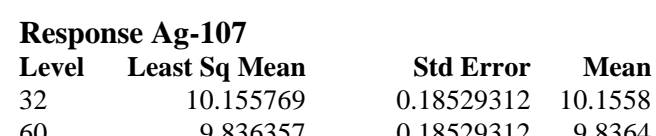

$\begin{array}{llll}60 & 9.836357 & 0.18529312 & 9.8364\end{array}$

Power Details

Alpha Sigma Delta Number Power

$\begin{array}{lllll}0.0500 & 0.585948 & 0.245909 & 20 & 0.4251\end{array}$

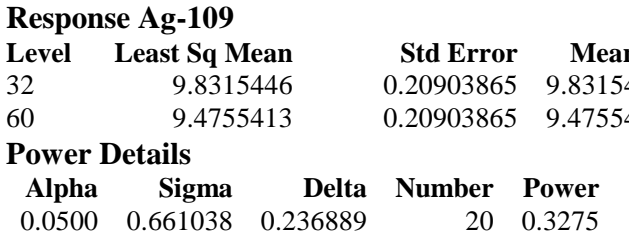

Response U-235

$\begin{array}{lrrr}\text { Level } & \text { Least Sq Mean } & \text { Std Error } & \text { Mean } \\ 32 & 55.264698 & 0.53459659 & 55.2647\end{array}$

$\begin{array}{llll}60 & 54.553741 & 0.53459659 & 54.5537\end{array}$

Power Details

$\begin{array}{rrrrr}\text { Alpha Sigma } & \text { Delta } & \text { Number } & \text { Power }\end{array}$

$\begin{array}{lllll}0.0500 & 1.690543 & 1.363844 & 20 & 0.9247\end{array}$

Response U-238

\begin{tabular}{|c|c|c|c|c|}
\hline Level & Least Sq Mea & & Std Error & Mean \\
\hline 32 & 13479.77 & & 185.49663 & 13479.8 \\
\hline 60 & 13439.69 & & 185.49663 & 13439.7 \\
\hline \multicolumn{5}{|c|}{ Power Details } \\
\hline Alpha & Sigma & Delta & Number & Power \\
\hline 0.0500 & $586.5918 \quad 3$ & 35.9923 & 20 & 0.6755 \\
\hline
\end{tabular}

Response Pu-239

$\begin{array}{lrrr}\text { Level } & \text { Least Sq Mean } & \text { Std Error } & \text { Mean } \\ 32 & 21.821460 & 0.32209931 & 21.8215 \\ 60 & 20.901971 & 0.32209931 & 20.9020\end{array}$

$\begin{array}{llll}60 & 20.901971 & 0.32209931 & 20.9020\end{array}$

Power Details

Alpha Sigma Delta Number Power

$\begin{array}{lllll}0.0500 & 1.018567 & 0.522549 & 20 & 0.5807\end{array}$ 
WSRC-TR-2001-00565

Revision 0

Page 59 of 83

November 26, 2001

\section{Appendix (continued)}

\section{Exhibit A9. Statistical Analyses of the Mass Concentrations (micrograms per gram of slurry) with Potential Outliers Removed by Type of Composite and ICP Calibration Block (continued)}

Response Tc-99

LIMS Number Removed From this Analysis: 300166377.

Actual by Predicted Plot

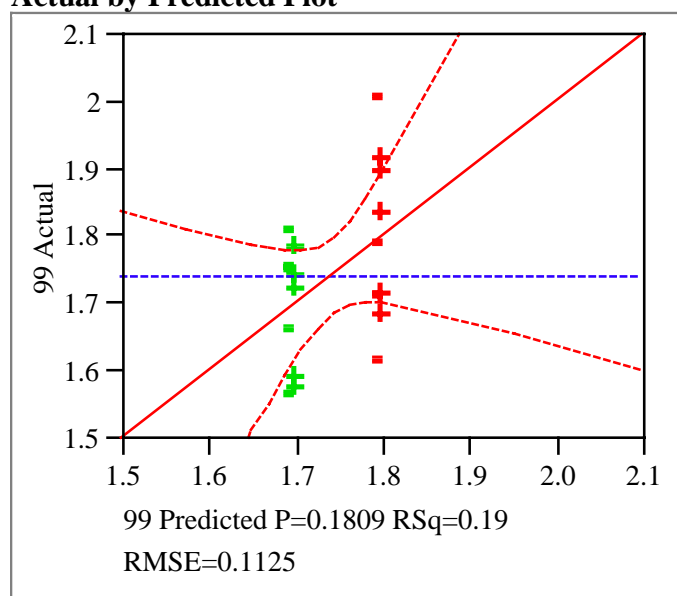

Summary of Fit

RSquare $\quad 0.192426$

RSquare Adj $\quad 0.091479$

Root Mean Square Error $\quad 0.112514$

Mean of Response 1.741659

Observations (or Sum Wgts)

19

Analysis of Variance

$\begin{array}{lrrrr}\text { Source } & \text { DF } & \text { Sum of Squares } & \text { Mean Square } & \text { F Ratio } \\ \text { Model } & 2 & 0.04826334 & 0.024132 & 1.9062\end{array}$

$\begin{array}{lllll}\text { Error } & 16 & 0.20255176 & 0.012659 & \text { Prob > F }\end{array}$

$\begin{array}{llll}\text { C. Total } & 18 & 0.25081510 & 0.1809\end{array}$

Lack Of Fit

Source DF Sum of Squares Mean Square F Ratio

$\begin{array}{lllll}\text { Lack Of Fit } & 1 & 0.00330265 & 0.003303 & 0.2486\end{array}$

$\begin{array}{lllll}\text { Pure Error } & 15 & 0.19924911 & 0.013283 & \text { Prob > F }\end{array}$

$\begin{array}{lllr}\text { Total Error } & 16 & 0.20255176 & 0.6253\end{array}$

Max RSq

0.2056

Parameter Estimates

Term

Intercept

Composite Type[32]

Calibration[1]

Effect Tests

Source

Composite Type

Calibration

$\begin{array}{rrrr}\text { Estimate } & \text { Std Error } & \text { t Ratio } & \text { Prob }>|\mathbf{t}| \\ 1.7441857 & 0.025888 & 67.37 & <.0001 \\ 0.0502911 & 0.025888 & 1.94 & 0.0699 \\ -0.00229 & 0.025888 & -0.09 & 0.9306\end{array}$

Nparm DF Sum of Squares F Ratio Prob > F $\begin{array}{lll}0.04777370 & 3.7737 & 0.0699\end{array}$ $\begin{array}{lll}0.00009902 & 0.0078 & 0.9306\end{array}$
Residual by Predicted Plot

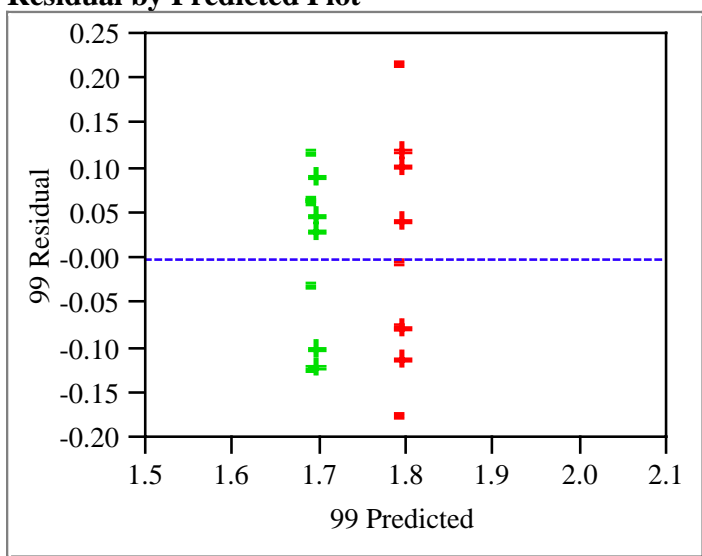

\section{Composite Type}

Least Squares Means Table

Level Least Sq Mean Std Error Mean

$\begin{array}{lrrrr}32 & 1.7944768 & 0.03761494 & 1.79473\end{array}$

$\begin{array}{lllll}60 & 1.6938946 & 0.03558017 & 1.69389\end{array}$ 
WSRC-TR-2001-00565

Revision 0

Page 60 of 83

November 26, 2001

\section{Appendix (continued)}

\section{Exhibit A9. Statistical Analyses of the Mass Concentrations (micrograms per gram of slurry) with Potential Outliers Removed by Type of Composite and ICP Calibration Block (continued)}

Response Ru-101

LIMS Number Removed From this Analysis: 300166395.

Actual by Predicted Plot

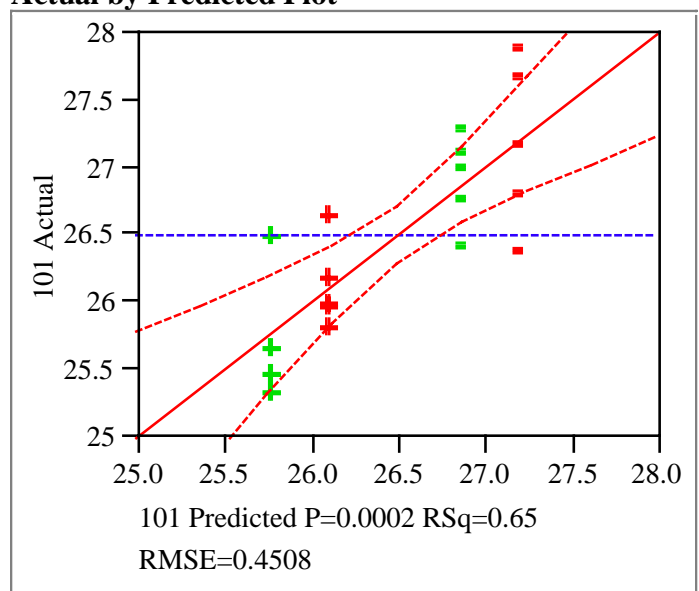

Summary of Fit

RSquare $\quad 0.647865$

RSquare Adj $\quad 0.603849$

Root Mean Square Error $\quad 0.450796$

Mean of Response 26.50571

Observations (or Sum Wgts)

Analysis of Variance

$\begin{array}{lrrrr}\text { Source } & \text { DF } & \text { Sum of Squares } & \text { Mean Square } & \text { F Ratio } \\ \text { Model } & 2 & 5.9821255 & 2.99106 & 14.7186\end{array}$

$\begin{array}{lrrrr}\text { Model } & 2 & 5.9821255 & 2.99106 & 14.7186 \\ \text { Error } & 16 & 3.2514671 & 0.20322 & \text { Prob > F }\end{array}$

$\begin{array}{llll}\text { C. Total } & 18 & 9.2335926 & 0.0002\end{array}$

Lack Of Fit

Source DF Sum of Squares Mean Square F Ratio

$\begin{array}{lllll}\text { Lack Of Fit } & 1 & 0.0157514 & 0.015751 & 0.0730\end{array}$

$\begin{array}{lllll}\text { Pure Error } & 15 & 3.2357156 & 0.215714 & \text { Prob > F }\end{array}$

$\begin{array}{llll}\text { Total Error } & 16 & 3.2514671 & 0.7907\end{array}$

Max RSq

0.6496

Parameter Estimates

Term

Intercept

Composite Type[32]

Calibration[1]

Effect Tests

Source

Composite Type

Calibration

$$
\begin{array}{rrrr}
\text { Estimate } & \text { Std Error } & \text { t Ratio } & \text { Prob }>|\mathbf{t}| \\
26.468458 & 0.103723 & 255.18 & <.0001 \\
0.1603234 & 0.103723 & 1.55 & 0.1417 \\
0.5475153 & 0.103723 & 5.28 & <.0001
\end{array}
$$

$\begin{array}{rrrrr}\text { Nparm } & \text { DF } & \text { Sum of Squares } & \text { F Ratio } & \text { Prob }>\text { F } \\ 1 & 1 & 0.4855120 & 2.3891 & 0.1417 \\ 1 & 1 & 5.6623787 & 27.8637 & <.0001\end{array}$

Residual by Predicted Plot



\section{Composite Type}

Least Squares Means Table

$\begin{array}{lrrr}\text { Level } & \text { Least Sq Mean } & \text { Std Error } & \text { Mean } \\ 32 & 26.628781 & 0.14255409 & 26.6288 \\ 60 & 26.308134 & 0.15070652 & 26.3690\end{array}$


WSRC-TR-2001-00565

Revision 0

Page 61 of 83

November 26, 2001

\section{Appendix (continued)}

\section{Exhibit A9. Statistical Analyses of the Mass Concentrations (micrograms per gram of slurry) with Potential Outliers Removed by Type of Composite and ICP Calibration Block (continued)}

Response Ru-102

\section{LIMS Number Removed From this Analysis:}

300166395.

Actual by Predicted Plot

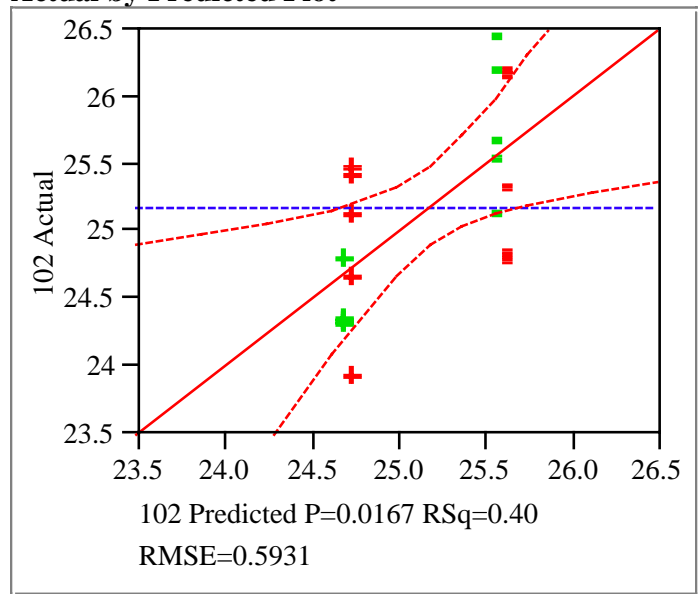

Summary of Fit

RSquare

0.400465

RSquare Adj

0.325523

Root Mean Square Error

0.59308

25.17134

Mean of Response

Observations (or Sum Wgts)

Analysis of Variance

Source DF Sum of Squares

Model $22 \quad 3.7592063$

$\begin{array}{lll}\text { Error } & 16 & 5.6278991\end{array}$

C. Total $18 \quad 9.3871054$

19

Lack Of Fit

Source

Lack Of Fit

DF Sum of Squares

Pure Error

Total Error

1
15
16

0.7745708

4.8533283

5.6278991

Mean Square 0.774571 0.323555

Square

1.87960

0.0167

Parameter Estimates

Term

Intercept

Composite Type[32]

Calibration[1]

Effect Tests

Source

Composite Type

Calibration

$\begin{array}{rrrrr} & \begin{array}{r}\text { Estimate } \\ \text { Std Error }\end{array} & \mathbf{t} \text { Ratio } & \text { Prob }>|\mathbf{t}| \\ 25.146651 & 0.136461 & 184.28 & <.0001 \\ & 0.0228915 & 0.136461 & 0.17 & 0.8689 \\ & 0.446109 & 0.136461 & 3.27 & 0.0048 \\ \text { Nparm } & \text { DF } & \text { Sum of Squares } & \text { F Ratio } & \text { Prob }>\text { F } \\ 1 & 1 & 0.0098982 & 0.0281 & 0.8689 \\ 1 & 1 & 3.7591385 & 10.6872 & 0.0048\end{array}$

Residual by Predicted Plot

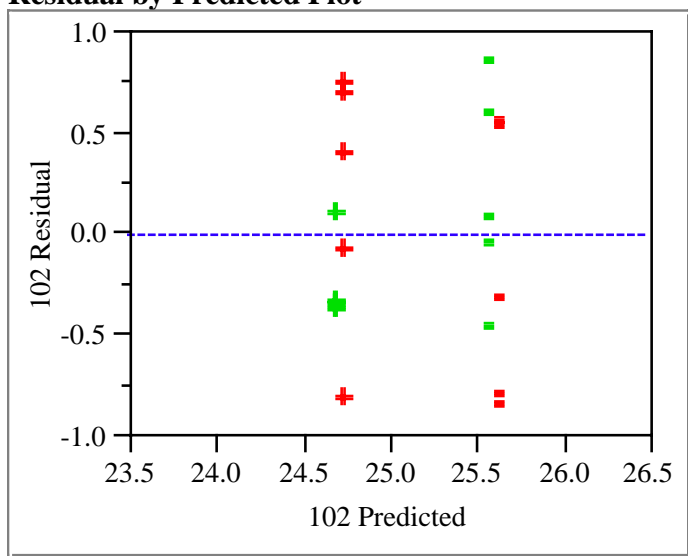

Composite Type

Least Squares Means Table

Level Least Sq Mean Std Error Mean

$\begin{array}{llll}32 & 25.169542 & 0.18754831 & 25.1695\end{array}$

$\begin{array}{llll}60 & 25.123759 & 0.19827388 & 25.1733\end{array}$ 
WSRC-TR-2001-00565

Revision 0

Page 62 of 83

November 26, 2001

\section{Appendix (continued)}

\section{Exhibit A9. Statistical Analyses of the Mass Concentrations (micrograms per gram of slurry) with Potential Outliers Removed by Type of Composite and ICP Calibration Block (continued)}

Response Rh-103

LIMS Number Removed From this Analysis: 300166395.

Actual by Predicted Plot



Summary of Fit

RSquare $\quad 0.625906$

RSquare Adj $\quad 0.579144$

Root Mean Square Error $\quad 0.336713$

Mean of Response 16.79628

Observations (or Sum Wgts) 19

Analysis of Variance

Source DF Sum of Squares Mean Square F Ratio

Model $2 \quad 3.0350625$

$\begin{array}{lll}\text { Error } \quad 16 & 1.8140096\end{array}$

C. Total $18 \quad 4.8490721$

1.51753

0.11338 Prob $>$ F

Lack Of Fit

Source

Lack Of Fit

Pure Error

DF Sum of Squares

$\begin{array}{rr}1 & 0.0103239 \\ 15 & 1.8036857 \\ 16 & 1.8140096\end{array}$

Mean Square 0.010324

0.120246

1.8140096

Total Error

Parameter Estimates

Term

Intercept

Composite Type[32]

Calibration[1]

Effect Tests

Source

Composite Type

Calibration

$\begin{array}{rrrrr}\text { Nparm } & \text { DF } & \text { Sum of Squares } & \text { F Ratio } & \text { Prob > F } \\ 1 & 1 & 0.0370295 & 0.3266 & 0.5756 \\ 1 & 1 & 3.0258580 & 26.6888 & <.0001\end{array}$

Residual by Predicted Plot

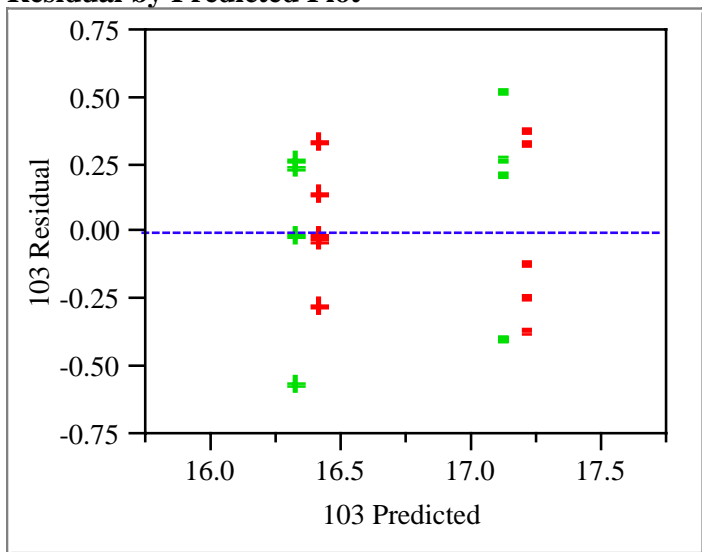

Composite Type

Least Squares Means Table

Level Least Sq Mean Std Error Mean

$\begin{array}{llll}32 & 16.817161 & 0.10647798 & 16.8172\end{array}$

$\begin{array}{llll}60 & 16.728609 & 0.11256727 & 16.7731\end{array}$ 
WSRC-TR-2001-00565

Revision 0

Page 63 of 83

November 26, 2001

\section{Appendix (continued)}

\section{Exhibit A9. Statistical Analyses of the Mass Concentrations (micrograms per gram of slurry) with Potential Outliers Removed by Type of Composite and ICP Calibration Block (continued)}

Response Ru-104

LIMS Number Removed From this Analysis:

300166395.

Actual by Predicted Plot

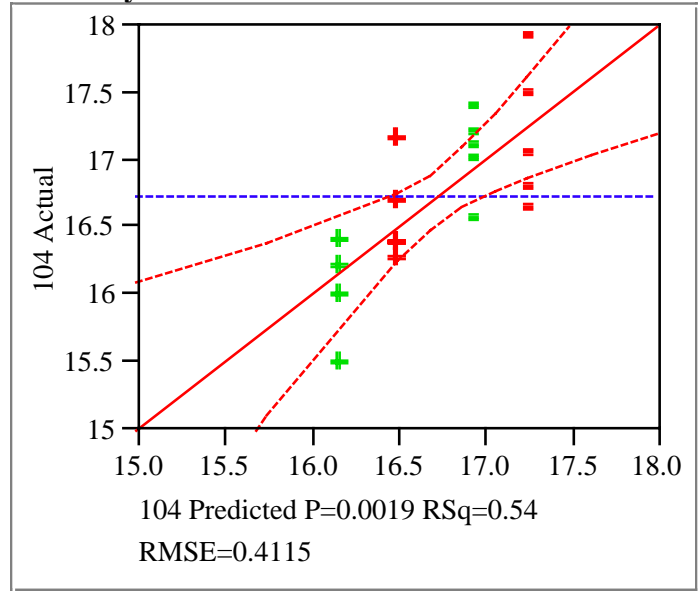

Summary of Fit

RSquare $\quad 0.541771$

RSquare Adj $\quad 0.484492$

Root Mean Square Error $\quad 0.41146$

Mean of Response 16.72942

Observations (or Sum Wgts) 19

Analysis of Variance

Source DF Sum of Squares Mean Square F Ratio

Model $22 \quad 3.2026425$

$\begin{array}{lll}\text { Error } & 16 & 2.7087938\end{array}$

C. Total $18 \quad 5.9114363$

1.60132

0.16930 Prob $>$ F

0.0019

Lack Of Fit

Source

Lack Of Fit

DF Sum of Squares

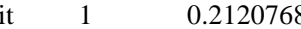

Pure Error $\quad 15 \quad 2.4967170$

Total Error $\quad 16 \quad 2.7087938$

Mean Square 0.212077

0.166448

F Ratio

1.2741

Prob > F

0.2767

Max RSq

0.5776

Parameter Estimates

Term

Intercept

Composite Type[32]

Calibration[1]

Effect Tests

Source

Composite Type

Calibration
Residual by Predicted Plot

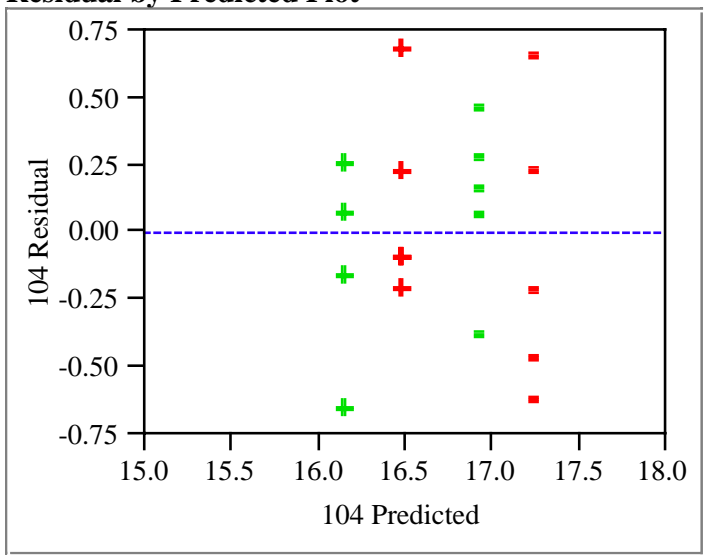

Composite Type

Least Squares Means Table

Level Least Sq Mean Std Error Mean

$\begin{array}{llll}32 & 16.862353 & 0.13011518 & 16.8624\end{array}$

$\begin{array}{llll}60 & 16.538721 & 0.13755625 & 16.5817\end{array}$ 
WSRC-TR-2001-00565

Revision 0

Page 64 of 83

November 26, 2001

\section{Appendix (continued)}

\section{Exhibit A9. Statistical Analyses of the Mass Concentrations (micrograms per gram of slurry) with Potential Outliers Removed by Type of Composite and ICP Calibration Block (continued)}

Response Pd-105

\section{LIMS Numbers Removed From this Analysis:}

300166389 and 300166394.

Actual by Predicted Plot



\section{Summary of Fit}

RSquare

0.446161

RSquare Adj

0.372316

Root Mean Square Error

0.110359

Mean of Response

1.253679

Observations (or Sum Wgts)

Analysis of Variance

Source DF Sum of Squares

Model 20.14716838

Error $\quad 15 \quad 0.18268613$

$\begin{array}{lll}\text { C. Total } & 17 & 0.32985451\end{array}$

18

Lack Of Fit

Source

Lack Of Fit

DF Sum of Squares

-0.02799556
-1

Pure Error $\quad 14 \quad 0.15469057$

Total Error $\quad 15 \quad 0.18268613$

Mean Square F Ratio $0.073584 \quad 6.0419$ 0.012179 Prob $>$ F

0.0119

Mean Square 0.027996 0.011049

\section{F Ratio}

$$
2.5337
$$

Prob > F

0.1338

Max RSq

0.5310

Parameter Estimates

Term

Intercept

Composite Type[32]

Calibration[1]

Effect Tests

Source

Composite Type

Calibration
Estimate

\subsection{3}

$-0.027739$

0.083255

Std Error

0.026381

0.026381

0.026381
Npa



D
Sum of Squares

t Ratio

$<.0001$

$\begin{array}{ll}3.16 & 0.0065\end{array}$

Composite Type

Least Squares Means Table

Level Least Sq Mean Std Error Mean

$\begin{array}{llll}32 & 1.2197710 & 0.03489853 & 1.21977\end{array}$

$\begin{array}{llll}60 & 1.2752495 & 0.03957122 & 1.29606\end{array}$

Residual by Predicted Plot

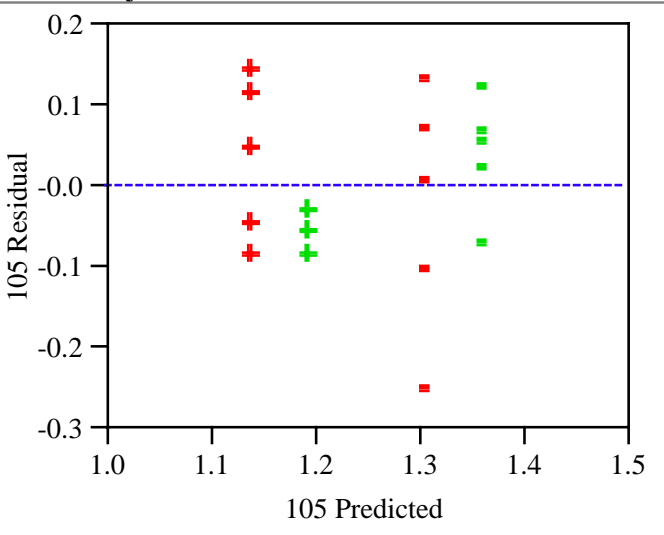


WSRC-TR-2001-00565

Revision 0

Page 65 of 83

November 26, 2001

\section{Appendix (continued)}

\section{Exhibit A9. Statistical Analyses of the Mass Concentrations (micrograms per gram of slurry) with Potential Outliers Removed by Type of Composite and ICP Calibration Block (continued)}

Response Ag-107

\section{LIMS Number Removed From this Analysis:}

300166385.

Actual by Predicted Plot

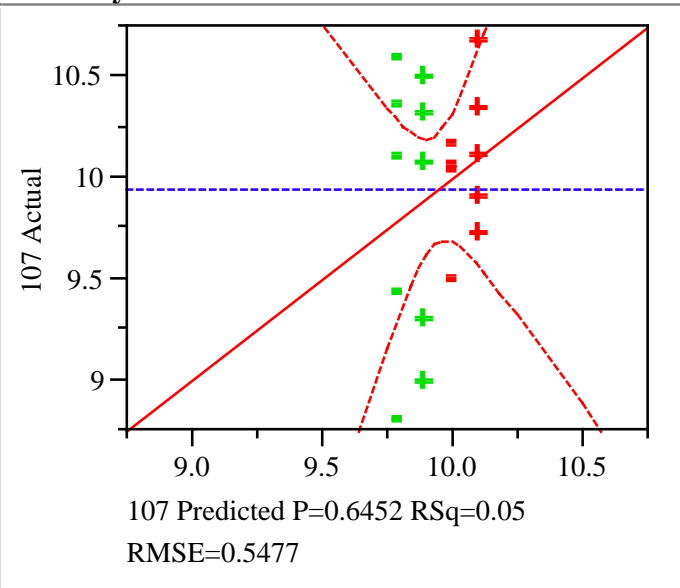

Summary of Fit

RSquare

0.053305

RSquare Adj

Root Mean Square Error

Mean of Response

Observations (or Sum Wgts)

Analysis of Variance

Source DF Sum of Squares

Model $2 \quad 0.2702325$

$\begin{array}{lll}\text { Error } & 16 & 4.7993223\end{array}$

C. Total $18 \quad 5.0695548$

$-0.06503$

0.547684

9.938083

19

Lack Of Fit

Source

Lack Of Fit

DF Sum of Squares

Pure Error

Total Error

$$
\begin{array}{rr}
1 & 0.0651035 \\
15 & 4.7342188 \\
16 & 4.7993223
\end{array}
$$

16

Mean Square 0.135116

0.299958 Prob $>$ F

0.6452

Mean Square F Ratio $\begin{array}{ll}0.065103 & 0.2063\end{array}$ 0.315615 Prob $>$ F 0.6562

Max RSq 0.0661

Parameter Estimates

Term

Intercept

Composite Type[32]

Calibration[1]

Effect Tests

Source

Composite Type

Calibration

Npar

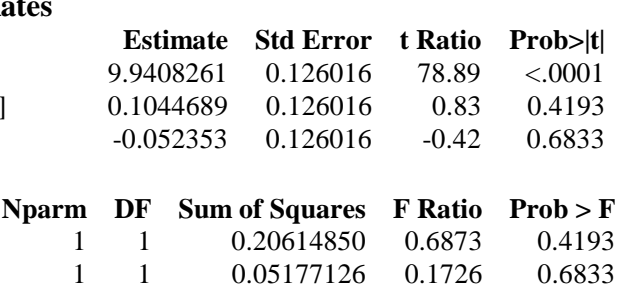

Residual by Predicted Plot



Composite Type

Least Squares Means Table

$\begin{array}{lrrr}\text { Level } & \text { Least Sq Mean } & \text { Std Error } & \text { Mean } \\ 32 & 10.045295 & 0.18309746 & 10.0511 \\ 60 & 9.836357 & 0.17319285 & 9.8364\end{array}$


WSRC-TR-2001-00565

Revision 0

Page 66 of 83

November 26, 2001

\section{Appendix (continued)}

\section{Exhibit A9. Statistical Analyses of the Mass Concentrations (micrograms per gram of slurry) with Potential Outliers Removed by Type of Composite and ICP Calibration Block (continued)}

Response Ag-109

\section{LIMS Number Removed From this Analysis:}

300166385.

Actual by Predicted Plot

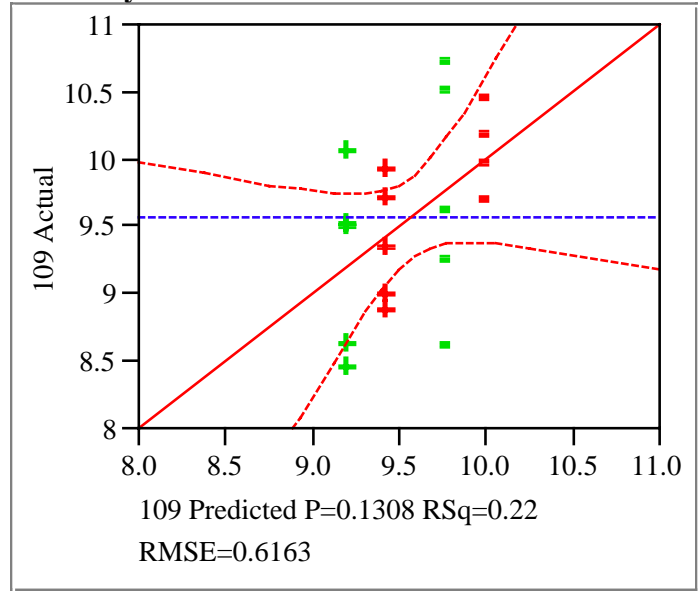

Summary of Fit

RSquare

RSquare Adj

Root Mean Square Error

Mean of Response

Observations (or Sum Wgts)

Analysis of Variance

Source DF Sum of Squares

Model $2 \quad 1.7598605$

$\begin{array}{lll}\text { Error } & 16 & 6.0776271\end{array}$

$\begin{array}{lll}\text { C. Total } & 18 & 7.8374876\end{array}$

0.224544

0.127612

0.616321

9.569264

19

Lack Of Fit

Source

Lack Of Fit

Pure Error

DF Sum of Squares

$1 \quad 0.0424341$

Total Error

6.0351930

6.0776271

Mean Square 0.879930

0.379852 Prob $>$ F

0.1308

Mean Square 0.042434 0.402346

F Ratio

0.1055

Prob > F

0.7499

Max RSq

0.2300

Parameter Estimates

Term

Intercept

Composite Type[32]

Calibration[1]

Effect Tests

Source

Composite Type

Calibration

$\begin{array}{rrrr}\text { Estimate } & \text { Std Error } & \text { t Ratio } & \text { Prob }>|\mathbf{t}| \\ 9.5905096 & 0.141809 & 67.63 & <.0001 \\ 0.1149683 & 0.141809 & 0.81 & 0.4294 \\ 0.2887072 & 0.141809 & 2.04 & 0.0587\end{array}$

Nparm DF Sum of Squares F Ratio Prob > F $\begin{array}{lll}0.2496678 & 0.6573 & 0.4294\end{array}$ $\begin{array}{lll}1.5744236 & 4.1448 & 0.0587\end{array}$
Residual by Predicted Plot

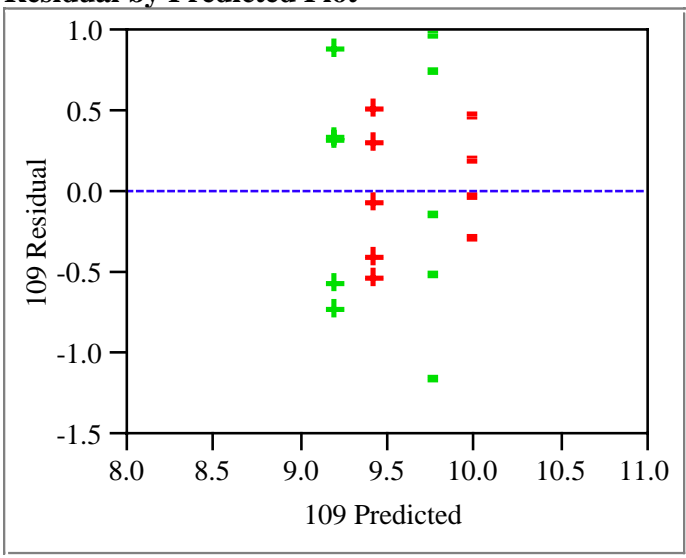

Composite Type

Least Squares Means Table

Level Least Sq Mean Std Error Mean

$\begin{array}{llll}32 & 9.7054779 & 0.20604372 & 9.67340\end{array}$

$\begin{array}{llll}60 & 9.4755413 & 0.19489784 & 9.47554\end{array}$ 
WSRC-TR-2001-00565

Revision 0

Page 67 of 83

November 26, 2001

\section{Appendix (continued)}

\section{Exhibit A9. Statistical Analyses of the Mass Concentrations (micrograms per gram of slurry) with Potential Outliers Removed by Type of Composite and ICP Calibration Block (continued)}

Response U-235

\section{LIMS Number Removed From this Analysis:}

300166395.

Actual by Predicted Plot



\section{Summary of Fit}

RSquare

0.786503

RSquare Adj

Root Mean Square Error

Mean of Response

Observations (or Sum Wgts)

Analysis of Variance

Source DF Sum of Squares

Model $22 \quad 53.102560$

$\begin{array}{lll}\text { Error } & 16 & 14.414763\end{array}$

$\begin{array}{lll}\text { C. Total } & 18 & 67.517323\end{array}$

0.759816

0.949169

55.14029

19

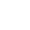

Mean Square F Ratio

$26.5513 \quad 29.4712$

0.9009 Prob > F

$<.0001$

Lack Of Fit

Source

Lack Of Fit

Pure Error

$\begin{array}{rr}\text { DF } & \text { Sum of Squares } \\ 1 & 0.032772\end{array}$

14.381991

Mean Square 0.032772

0.958799

F Ratio

0.0342

Prob > F

0.8558

Max RSq

0.7870

Parameter Estimates

Term

Intercept

Composite Type[32]

Calibration[1]

Effect Tests

Source

Composite Type

Calibration
Residual by Predicted Plot



Composite Type

Least Squares Means Table

Level Least Sq Mean Std Error Mean

$\begin{array}{llll}32 & 55.264698 & 0.30015374 & 55.2647\end{array}$

$\begin{array}{llll}60 & 55.187777 & 0.31731902 & 55.0021\end{array}$ 
WSRC-TR-2001-00565

Revision 0

Page 68 of 83

November 26, 2001

\section{Appendix (continued)}

\section{Exhibit A9. Statistical Analyses of the Mass Concentrations (micrograms per gram of slurry) with Potential Outliers Removed by Type of Composite and ICP Calibration Block (continued)}

Response U-238

LIMS Numbers Removed From this Analysis:

300166376 and 300166397.

Actual by Predicted Plot

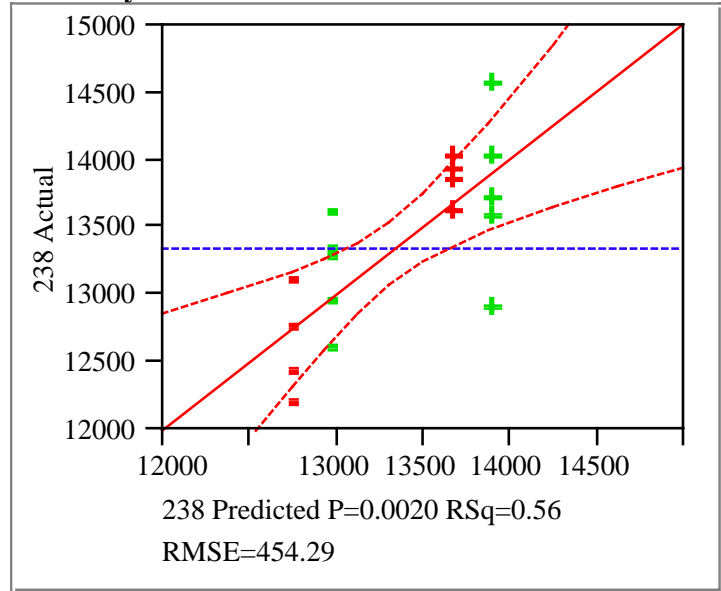

Summary of Fit

RSquare $\quad 0.564071$

RSquare Adj $\quad 0.505948$

Root Mean Square Error $\quad 454.2913$

Mean of Response 13341.32

Observations (or Sum Wgts) 18

Analysis of Variance

Source DF Sum of Squares Mean Square F Ratio

$\begin{array}{lllll}\text { Model } & 2 & 4005705.4 & 2002853 & 9.7047\end{array}$

$\begin{array}{lllrl}\text { Error } & 15 & 3095709.0 & 206381 & \text { Prob }>\text { F }\end{array}$

$\begin{array}{llll}\text { C. Total } & 17 & 7101414.3 & 0.0020\end{array}$

Lack Of Fit

Source DF Sum of Squares Mean Square F Ratio

$\begin{array}{lllll}\text { Lack Of Fit } & 1 & 437713.6 & 437714 & 2.3055\end{array}$

$\begin{array}{lllll}\text { Pure Error } & 14 & 2657995.3 & 189857 & \text { Prob }>\text { F }\end{array}$

$\begin{array}{llll}\text { Total Error } & 15 & 3095709.0 & 0.1512\end{array}$

Max RSq

0.6257

Parameter Estimates

Term

Intercept

Composite Type[32]

Calibration[1]

Effect Tests

Source

Composite Type

Calibration

$\begin{array}{rrrrr}\text { Nparm } & \text { DF } & \text { Sum of Squares } & \text { F Ratio } & \text { Prob > F } \\ 1 & 1 & 217749.4 & 1.0551 & 0.3206 \\ 1 & 1 & 3787955.9 & 18.3542 & 0.0007\end{array}$

Residual by Predicted Plot

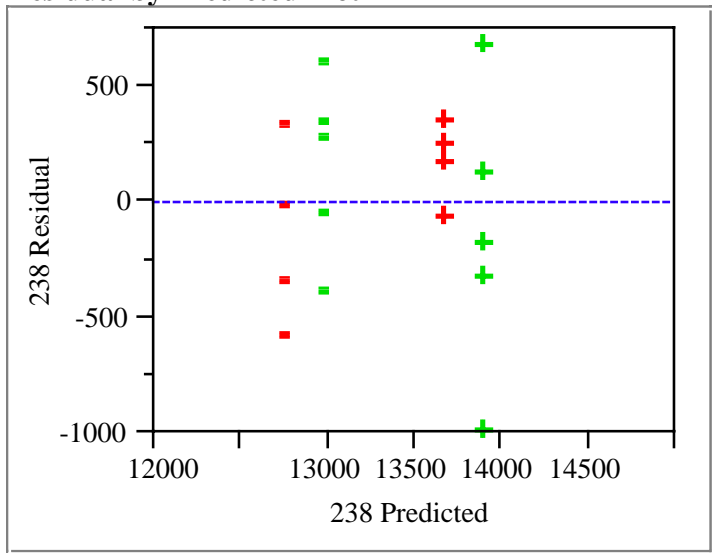

Composite Type

Least Squares Means Table

Level Least Sq Mean Std Error Mean

$\begin{array}{llll}32 & 13218.349 & 160.61623 & 13218.3\end{array}$

$\begin{array}{llll}60 & 13439.694 & 143.65953 & 13439.7\end{array}$ 
WSRC-TR-2001-00565

Revision 0

Page 69 of 83

November 26, 2001

\section{Appendix (continued)}

\section{Exhibit A9. Statistical Analyses of the Mass Concentrations (micrograms per gram of slurry) with Potential Outliers Removed by Type of Composite and ICP Calibration Block (continued)}

Response Pu-239

\section{LIMS Number Removed From this Analysis:}

300166395.

Actual by Predicted Plot

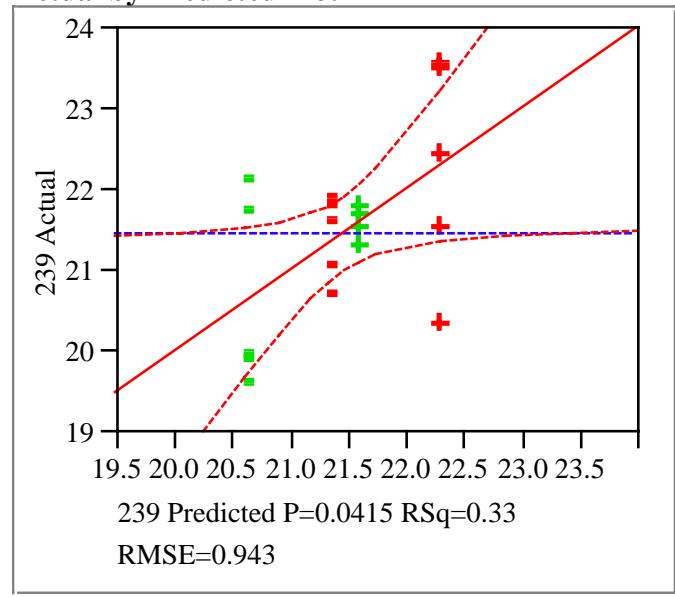

Summary of Fit

RSquare

0.328264

RSquare Adj

0.244298

0.942989

21.45615

Root Mquare Error

Observations (or Sum Wgts)

Analysis of Variance

Source DF Sum of Squares

Model $22 \quad 6.952780$

Error $\quad 16 \quad 14.227640$

$\begin{array}{lll}\text { C. Total } & 18 & 21.180420\end{array}$

19

Lack Of Fit

Source

Lack Of Fit

Pure Error

DF Sum of Squares

$\begin{array}{rr}1 & 0.004248 \\ 15 & 14.223392 \\ 16 & 14.227640\end{array}$

14.227640

Mean Square 3.47639 0.88923

F Ratio

3.9094
Prob $>$ F

0.0415

Mean Square 0.004248 0.948226

F Ratio 0.0045 Prob > F 0.9475 Max RSq 0.3285

Parameter Estimates

Term

Intercept

Composite Type[32]

Calibration[1]

Effect Tests

Source

Composite Type

Calibration

$\begin{array}{rrrrr}\text { Nparm } & \text { DF } & \text { Sum of Squares } & \text { F Ratio } & \text { Prob > F } \\ 1 & 1 & 2.4426288 & 2.7469 & 0.1169 \\ 1 & 1 & 4.1355471 & 4.6507 & 0.0466\end{array}$

Residual by Predicted Plot

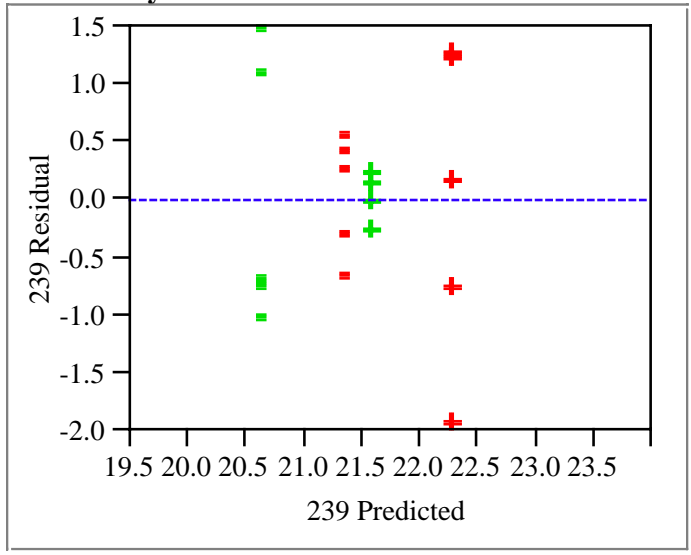

Composite Type

Least Squares Means Table

Level Least Sq Mean Std Error Mean

$\begin{array}{llll}32 & 21.821460 & 0.29819918 & 21.8215\end{array}$

$\begin{array}{llll}60 & 21.102250 & 0.31525267 & 21.0503\end{array}$ 
WSRC-TR-2001-00565

Revision 0

Page 70 of 83

November 26, 2001

\section{Appendix (continued)}

\section{Exhibit A10. Plots of the Cobalt and Cesium Radioactivity} (dpm per gram of slurry) by Type of Composite and ICP Calibration Block

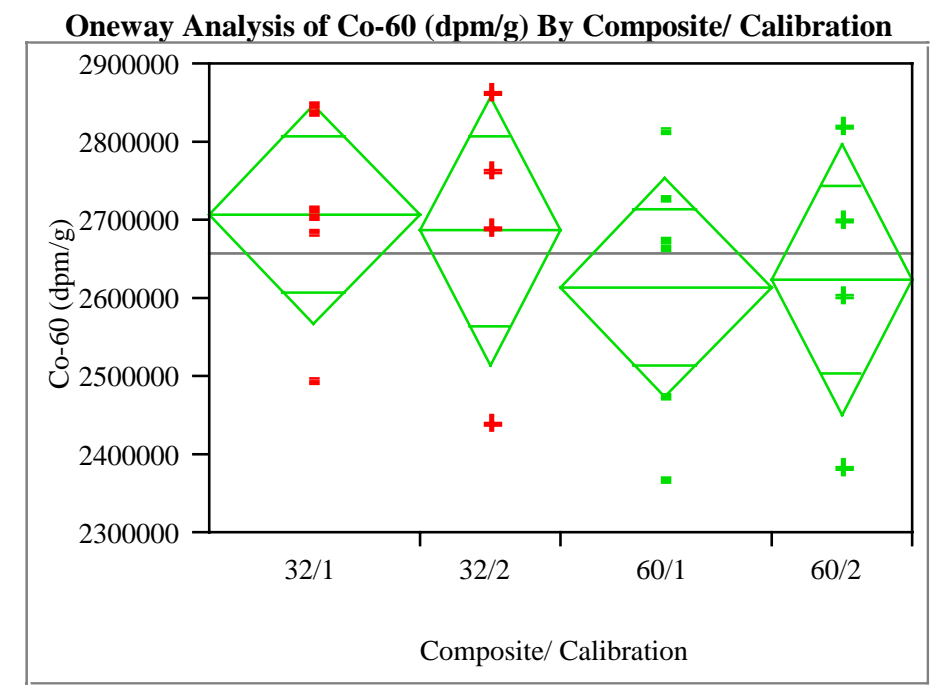

Oneway Analysis of Cs-137 (dpm/g) By Composite/ Calibration




WSRC-TR-2001-00565

Revision 0

Page 71 of 83

November 26, 2001

\section{Appendix (continued)}

\section{Exhibit A11. Statistical Analyses of the Cobalt and Cesium Radioactivity (dpm per gram of slurry) by Type of Composite and ICP Calibration Block}

Response Co-60 (dpm/g)

Actual by Predicted Plot

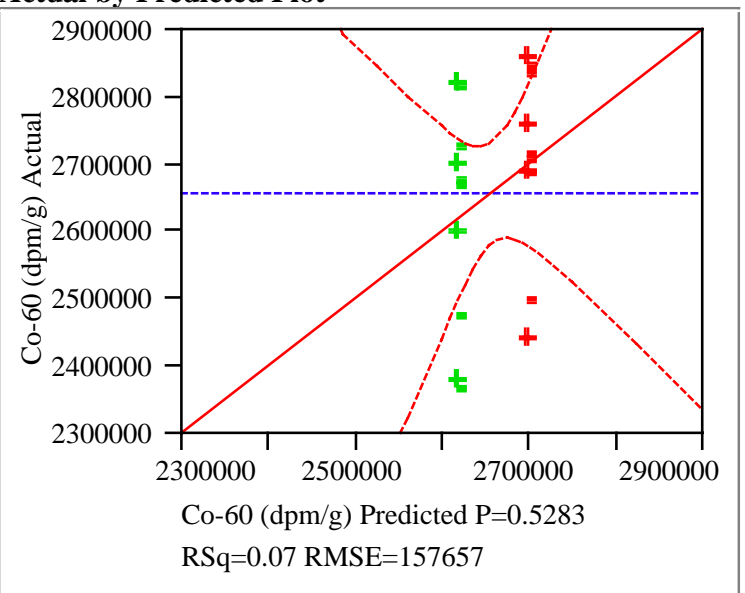

Residual by Predicted Plot



Summary of Fit

R-square is the portion of variation attributed to the model, between 0 and 1. Root Mean Squared Error "RMSE" estimates the standard deviation of the residual.

RSquare

0.07233

RSquare Adj $-0.03681$

Root Mean Square Error $\quad 157657.3$

Mean of Response 2659500

Observations (or Sum Wgts) 20

Analysis of Variance

The test that the whole model fits better than a simple mean, i.e. testing that all the parameters are zero except the intercept

Source DF Sum of Squares Mean Square F Ratio

Model $\quad 2 \quad 3.29458 \mathrm{e} 10 \quad 1.6473 \mathrm{e} 10 \quad 0.6627$

$\begin{array}{lllll}\text { Error } & 17 & 4.22549 \mathrm{e} 11 & 2.4856 \mathrm{e} 10 \quad \text { Prob }>\text { F }\end{array}$

$\begin{array}{llll}\text { C. Total } & 19 & 4.55495 \mathrm{e} 11 & 0.5283\end{array}$

Lack Of Fit

Using replicated points as the part of residual error that does not depend on the form of the model so that you can test for the adequacy of the form of the model.

Source

$$
\text { DF }
$$

Sum of Squares 1140833333

Mean Square

ean Square
$1.14083 \mathrm{e} 9$
$2.6338 \mathrm{e} 10$

\section{F Ratio}

0.0433

Pure Error

$4.21408 \mathrm{e} 11$

Prob > F 0.8378

Max RSq

Parameter Estimates

Term

Intercept

Composite Type[32]

Calibration[1]

Effect Tests

Source

Composite Type

Calibration

Nparm

$\begin{array}{rrrr}\text { Estimate } & \text { Std Error } & \text { t Ratio } & \text { Prob }>|\mathbf{t}| \\ 2658958.3 & 35980.2 & 73.90 & <.0001 \\ 40500 & 35253.25 & 1.15 & 0.2665 \\ 2708.3333 & 35980.2 & 0.08 & 0.9409\end{array}$

$\begin{array}{llll}2708.3333 & 35980.2 & 0.08 & 0.9409\end{array}$ 
WSRC-TR-2001-00565

Revision 0

Page 72 of 83

November 26, 2001

\section{Appendix (continued)}

\section{Exhibit A11. Statistical Analyses of the Cobalt and Cesium Radioactivity (dpm per gram of slurry) by Type of Composite and ICP Calibration Block (continued)}
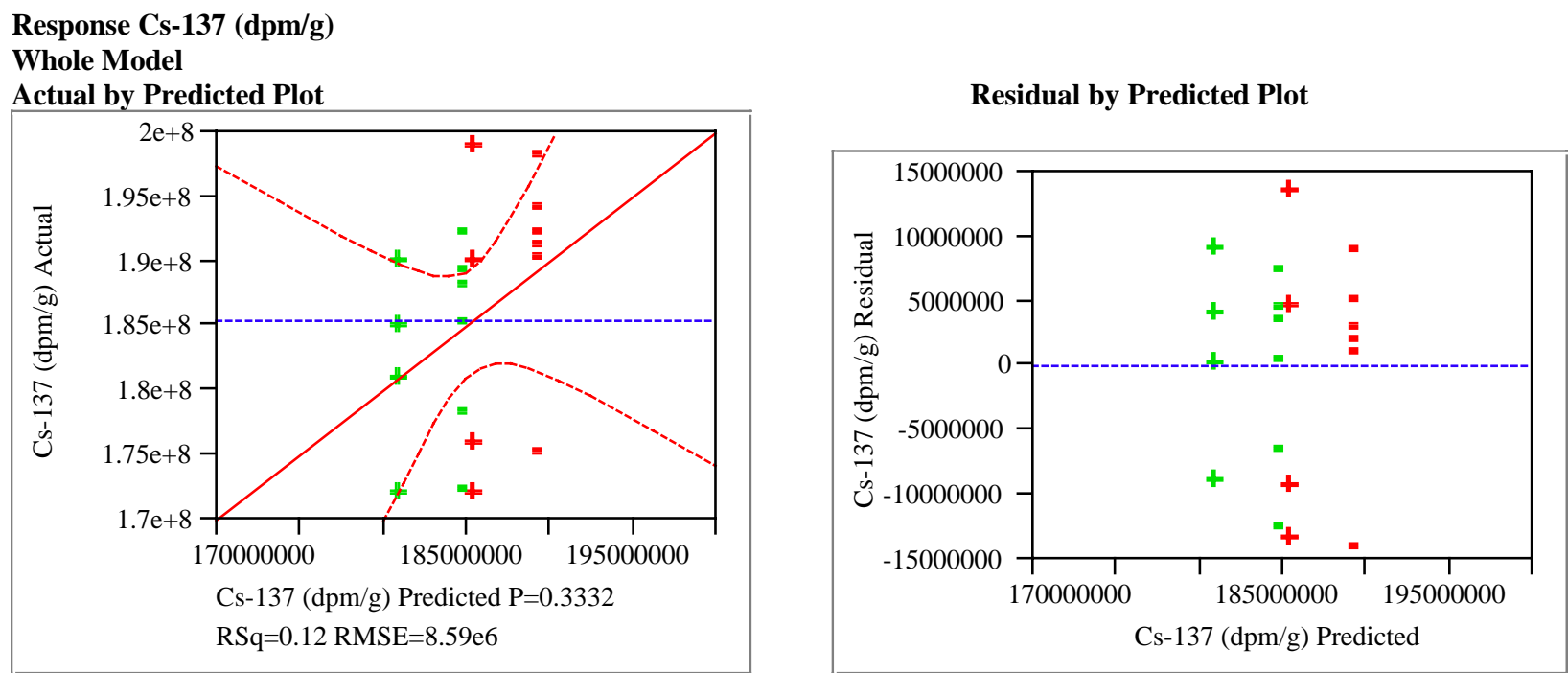

\section{Summary of Fit}

R-square is the portion of variation attributed to the model, between 0 and 1. Root Mean Squared Error "RMSE" estimates the standard deviation of the residual.
RSquare
0.121295
RSquare Adj
0.017918
Root Mean Square Error 8594201
Mean of Response $1.8545 \mathrm{e} 8$
Observations (or Sum Wgts)

Analysis of Variance

The test that the whole model fits better than a simple mean, i.e. testing that all the parameters are zero except the intercept

Source DF Sum of Squares Mean Square F Ratio

$\begin{array}{lllll}\text { Model } & 2 & 1.73325 \mathrm{e} 14 & 8.6663 \mathrm{e} 13 & 1.1733\end{array}$

$\begin{array}{lllll}\text { Error } & 17 & 1.25563 \mathrm{e} 15 & 7.386 \mathrm{e}+13 & \text { Prob }>\text { F }\end{array}$

$\begin{array}{llll}\text { C. Total } & 19 & 1.42895 \mathrm{e} 15 & 0.3332\end{array}$

\section{Lack Of Fit}

Using replicated points as the part of residual error that does not depend on the form of the model so that you can test for the adequacy of the form of the model.

Source

Lack Of Fit

Pure Error

DF Sum of Squares

$$
\begin{aligned}
& 1.6875 \mathrm{e}+13 \\
& 1.23875 \mathrm{e} 15
\end{aligned}
$$

Mean Square $1.6875 \mathrm{e} 13$
$7.7422 \mathrm{e} 13$

$7.7422 \mathrm{e} 13$

F Ratio

0.2180

Prob > F

0.6469

Max RSq

0.1331

Parameter Estimates

Term

Intercept

Composite Type[32]

Calibration[1]

Effect Tests

Source

Composite Type

Calibration

$\begin{array}{rrrr}\text { Estimate } & \text { Std Error } & \text { t Ratio } & \text { Prob }>|\mathbf{t}| \\ 185062500 & 1961349 & 94.35 & <.0001 \\ 2250000 & 1921722 & 1.17 & 0.2578 \\ 1937500 & 1961349 & 0.99 & 0.3371\end{array}$

$\begin{array}{rrrrr}\text { Nparm } & \text { DF } & \text { Sum of Squares } & \text { F Ratio } & \text { Prob > F } \\ 1 & 1 & 1.0125 \mathrm{e}+14 & 1.3708 & 0.2578 \\ 1 & 1 & 7.2075 \mathrm{e}+13 & 0.9758 & 0.3371\end{array}$


WSRC-TR-2001-00565

Revision 0

Page 73 of 83

November 26, 2001

\section{Appendix (continued)}

Exhibit A12. Sensitivity of Statistical Analyses of the Cobalt and Cesium Radioactivity (in dpm per gram of slurry) to 5\% Differences Due to Type of Composite

\begin{tabular}{|c|c|c|c|c|}
\hline \multicolumn{5}{|c|}{ Response Co-60 (dpm/g) } \\
\hline Level & Least Sq Mean & & rror & Mean \\
\hline 32 & 2699458.3 & & .277 & 2700000 \\
\hline 60 & 2618458.3 & & .277 & 2619000 \\
\hline \multicolumn{5}{|c|}{ Power Details } \\
\hline 0.0500 & 157657.3 & 65461.46 & 20 & 0.4177 \\
\hline \multicolumn{5}{|c|}{ Response Cs-137 (dpm/g) } \\
\hline Level & Least Sq Mean & & ror & Mean \\
\hline 32 & 187312500 & & 38.7 & 187700000 \\
\hline 60 & 182812500 & & 38.7 & 183200000 \\
\hline \multicolumn{5}{|c|}{ Power Details } \\
\hline Alpha & Sigma & Delta & Number & Power \\
\hline 0.0500 & 8594201 & 4570313 & 20 & 0.6113 \\
\hline
\end{tabular}


WSRC-TR-2001-00565

Revision 0

Page 74 of 83

November 26, 2001

\section{Appendix (continued)}

\section{Exhibit A13. Statistical Analyses of the Screened Cobalt and Cesium Radioactivity (dpm per gram of slurry) by Type of Composite and ICP Calibration Block}

Response Co-60 (dpm/g)

\section{LIMS Numbers Removed From this Analysis:}

$300166378,300166381,300166388,300166394$, and

300166397.

Actual by Predicted Plot

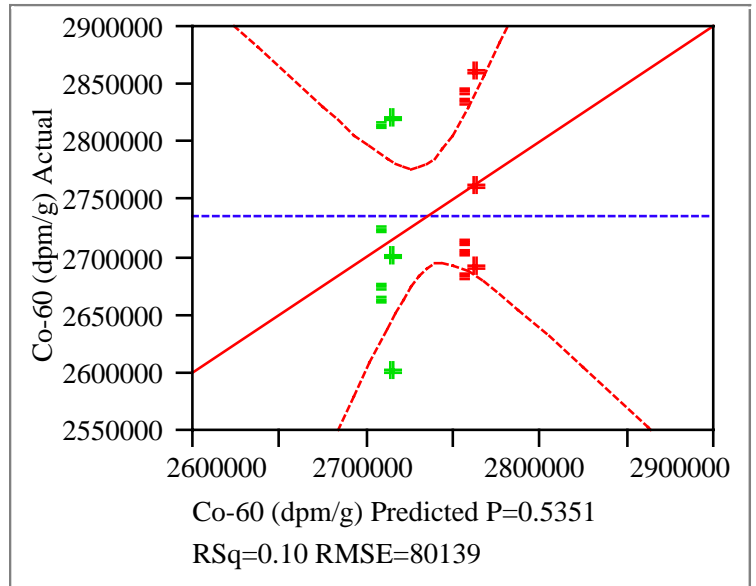

Summary of Fit

RSquare $\quad 0.098975$

RSquare Adj $\quad-0.0512$

Root Mean Square Error $\quad 80139.29$

Mean of Response 2736667

Observations (or Sum Wgts) 15

Analysis of Variance

Source DF $\quad$ Sum of Squares Mean Square $\quad$ F Ratio

$\begin{array}{lllll}\text { Model } & 2 & 8465671642 & 4.23284 \mathrm{e} 9 & 0.6591\end{array}$

$\begin{array}{lllll}\text { Error } & 12 & 7.70677 \mathrm{e} 10 & 6.42231 \mathrm{e} 9 & \text { Prob }>\text { F }\end{array}$

$\begin{array}{llrr}\text { C. Total } & 14 & 8.55333 \mathrm{e} 10 & 0.5351\end{array}$

Lack Of Fit

Source DF Sum of Squares

Lack Of Fit $\quad 1 \quad 620995025$

Pure Error $\quad 11 \quad 7.64467 \mathrm{e} 10$

Total Error $\quad 12 \quad 7.70677 \mathrm{e} 10$

Mean Square 0.0894

Prob > F 0.7706

Max RSq

0.1062

Parameter Estimates

Term

Intercept

Composite Type[32]

Calibration[1]

Effect Tests

Source

Composite Type

Calibration

N



Dparm Sum of Squares F Ratio Prob > F

$\begin{array}{rrrr}1 & 8437893864 & 1.3138 & 0.2740\end{array}$

$\begin{array}{llrrr}1 & 1 & 8437893864 & 1.3138 & 0.2740 \\ 1 & 1 & 105552594 & 0.0164 & 0.9001\end{array}$



\begin{tabular}{lrrr}
\hline \multicolumn{3}{l}{ Composite Type-Least Squares Means Table } \\
Level & Least Sq Mean & Std Error & Mean \\
32 & 2759427.9 & 28822.664 & 2758750 \\
60 & 2711815.9 & 30440.125 & 2711429
\end{tabular}


WSRC-TR-2001-00565

Revision 0

Page 75 of 83

November 26, 2001

\section{Appendix (continued)}

\section{Exhibit A13. Statistical Analyses of the Screened Cobalt and Cesium Radioactivity (dpm per gram of slurry) by Type of Composite and ICP Calibration Block (continued)}

Response Cs-137 (dpm/g)

\section{LIMS Numbers Removed From this Analysis:}

300166376 and 300166397.

Actual by Predicted Plot

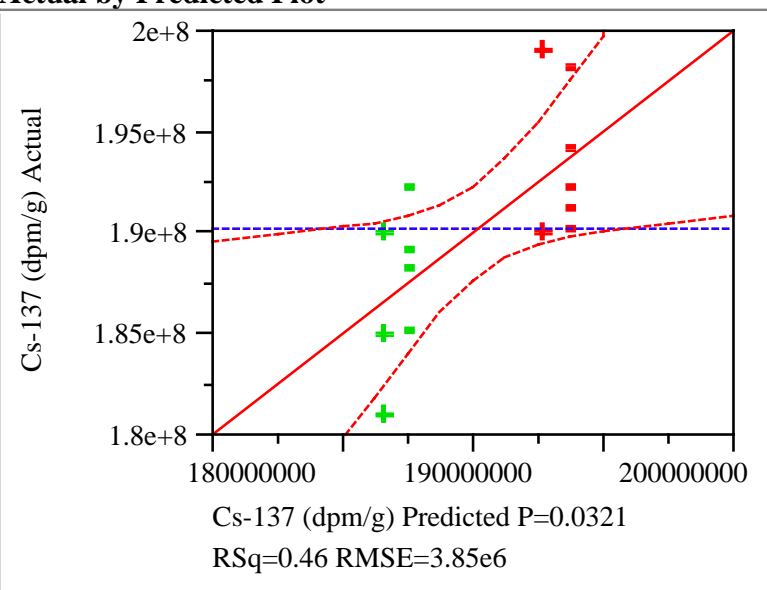

\section{Summary of Fit}

RSquare

RSquare Adj

0.464876

Root Mean Square Error

0.367581

3851049

Mean of Response

$1.9029 \mathrm{e} 8$

Observations (or Sum Wgts)

Analysis of Variance

$\begin{array}{lrrrr}\text { Model } & 2 & 1.41721 \mathrm{e} 14 & 7.086 \mathrm{e}+13 & 4.7780 \\ \text { Error } & 11 & 1.63136 \mathrm{e} 14 & 1.4831 \mathrm{e} 13 & \text { Prob > F } \\ \text { C. Total } & 13 & 3.04857 \mathrm{e} 14 & & 0.0321\end{array}$

Lack Of Fit

\section{Source}

Lack Of Fit

DF

Sum of Squares

$1.69697 \mathrm{e} 13$

$1.46167 \mathrm{e} 14$

Mean Square

$1.697 \mathrm{e}+13$

$1.4617 \mathrm{e} 13$

$1.63136 \mathrm{e} 14$

Total Error

11

$$
1.63136 \mathrm{e}
$$

Parameter Estimates

Term

Intercept

Composite Type[32]

Calibration[1]

Effect Tests

Source Nparm DF Sum of Squares F Ratio Prob > F

$\begin{array}{lllllll}\text { Composite Type } & 1 & 1 & 1.28864 \mathrm{e} 14 & 8.6890 & 0.0133\end{array}$

Calibration

$$
\begin{array}{rrrr}
\text { Estimate } & \text { Std Error } & \text { t Ratio } & \text { Prob }>|\mathbf{t}| \\
190136364 & 1075002 & 176.87 & <.0001 \\
3068181.8 & 1040866 & 2.95 & 0.0133 \\
522727.27 & 1086142 & 0.48 & 0.6398
\end{array}
$$

Residual by Predicted Plot

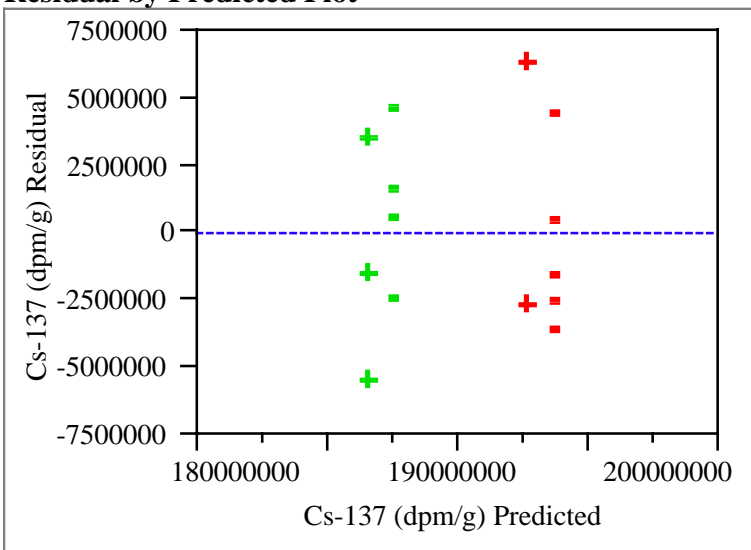

Composite Type-Least Squares Means Table Level Least Sq Mean Std Error Mean $\begin{array}{llrrr}32 & 193204545 & 1528180.2 & 193428571\end{array}$

$\begin{array}{llll}60 & 187068182 & 1463806.6 & 187142857\end{array}$


WSRC-TR-2001-00565

Revision 0

Page 76 of 83

November 26, 2001

\section{Appendix (continued)}

\section{Exhibit A14. Statistical Analyses of the Weight Percent Solids of the Supernate by Type of Composite}

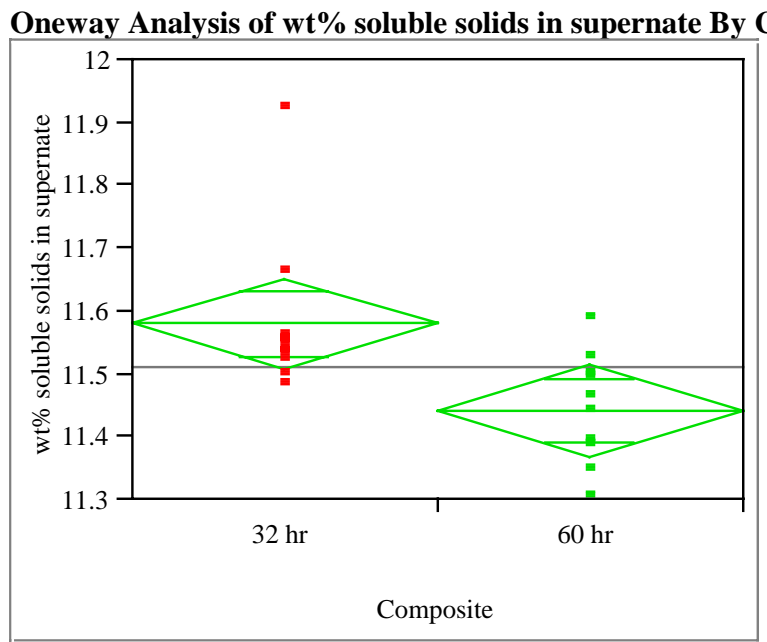



\begin{tabular}{lrrrrr}
\multicolumn{6}{l}{ Means for Oneway Anova } \\
Level & Number & $\begin{array}{r}\text { Mean } \\
\text { Sum Error }\end{array}$ & Lower 95\% & Upper 95\% \\
$32 \mathrm{hr}$ & 10 & 11.5802 & 0.03489 & 11.507 & 11.653 \\
$60 \mathrm{hr}$ & 10 & 11.4419 & 0.03489 & 11.369 & 11.515
\end{tabular}

Std Error uses a pooled estimate of error variance

Tests that the Variances are Equal

\begin{tabular}{|c|c|c|c|c|c|}
\hline Level & Count & Std Dev & $\begin{array}{r}\text { Mea } \\
\text { Dif to I }\end{array}$ & $\begin{array}{l}\text { Abs } \\
\text { lean }\end{array}$ & $\begin{array}{l}\text { MeanAbs } \\
\text { Dif to Median }\end{array}$ \\
\hline $32 \mathrm{hr}$ & 10 & 0.1292661 & 0.084 & 200 & 0.067400 \\
\hline $60 \mathrm{hr}$ & 10 & 0.0873797 & 0.070 & 000 & 0.07010 \\
\hline Test & & F Ratio & DFNum & DFDen & Prob $>$ F \\
\hline O’Brien & {$[.5]$} & 0.4387 & 1 & 18 & 0.5161 \\
\hline Brown- & Forsythe & 0.0047 & 1 & 18 & 0.9462 \\
\hline Leven & & 0.1783 & 1 & 18 & 0.6778 \\
\hline Bartlett & & 1.2755 & 1 & & 0.2587 \\
\hline
\end{tabular}

Welch Anova testing Means Equal, allowing Std Devs Not Equal

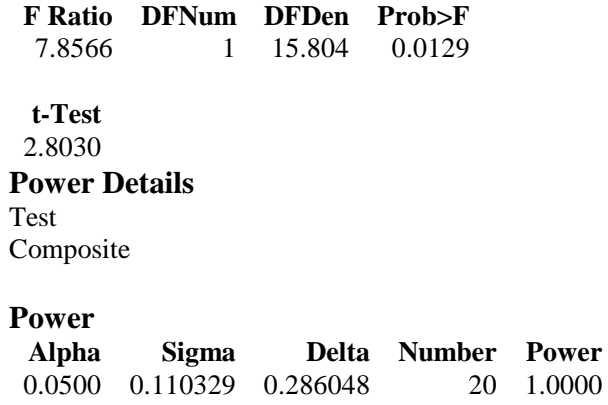


WSRC-TR-2001-00565

Revision 0

Page 77 of 83

November 26, 2001

\section{Appendix (continued)}

\section{Exhibit A15. Statistical Analyses of the Screened Weight Percent Solids of the Supernate by Type of Composite}

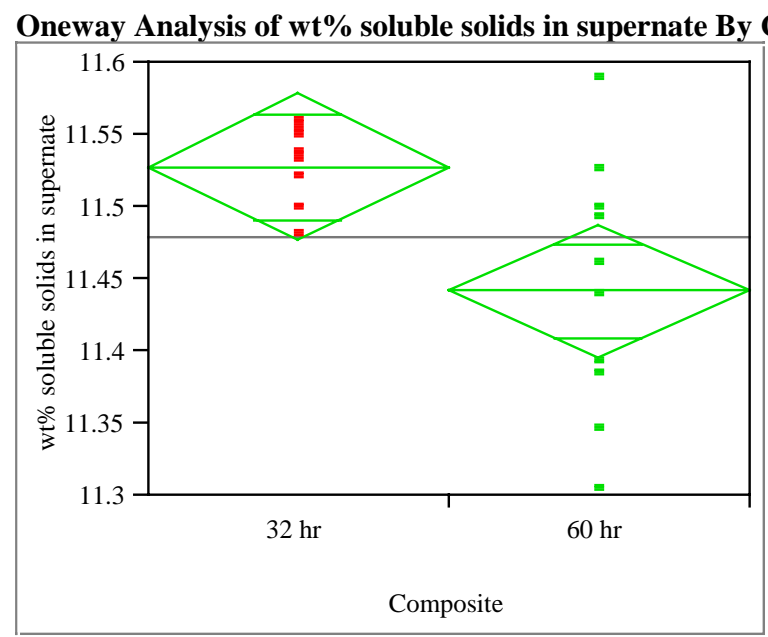

Oneway Anova

Summary of Fit

Rsquare

Adj Rsquare

Root Mean Square Error

0.306344

0.262991

0.067987

11.48

Observations (or Sum Wgts)

18

\section{t-Test}

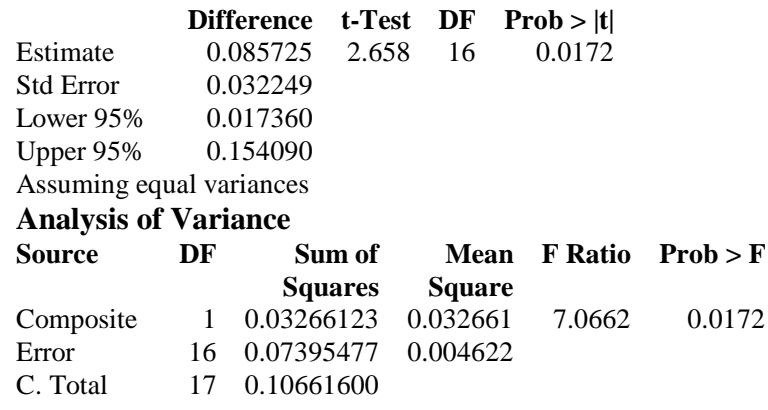

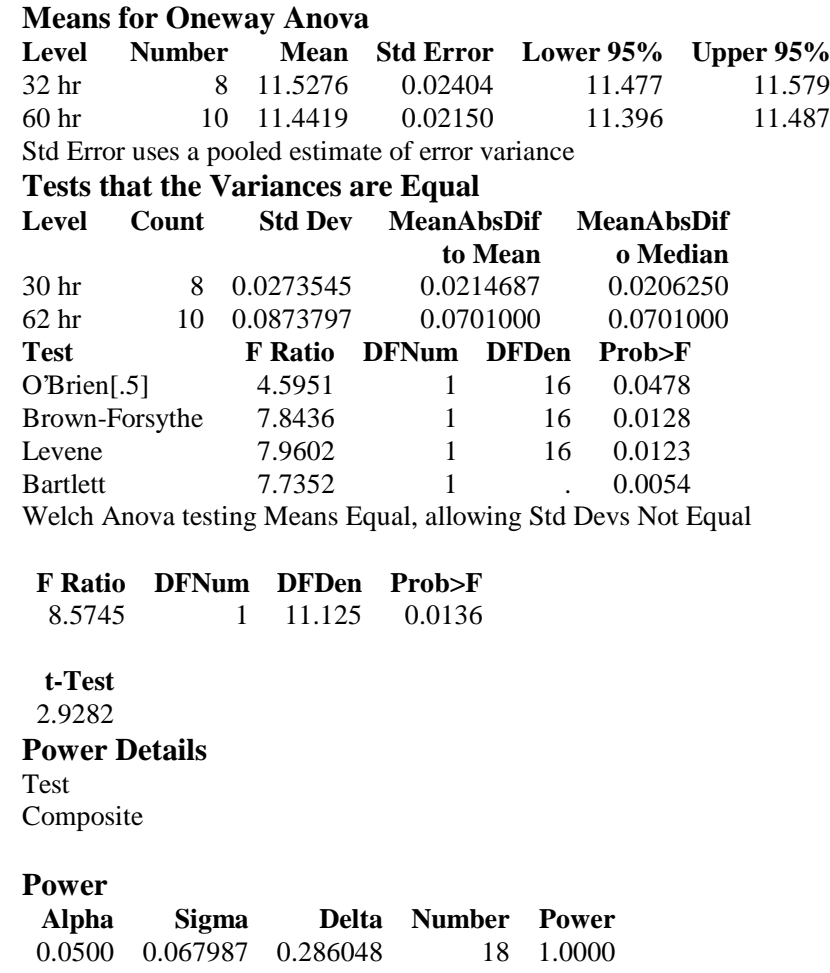


WSRC-TR-2001-00565

Revision 0

Page 78 of 83

November 26, 2001

\section{Appendix (continued)}

Exhibit A16. Plots of the Al and Na Concentrations (as grams/gram of supernate) in the Supernate by Type of Composite and ICP Calibration

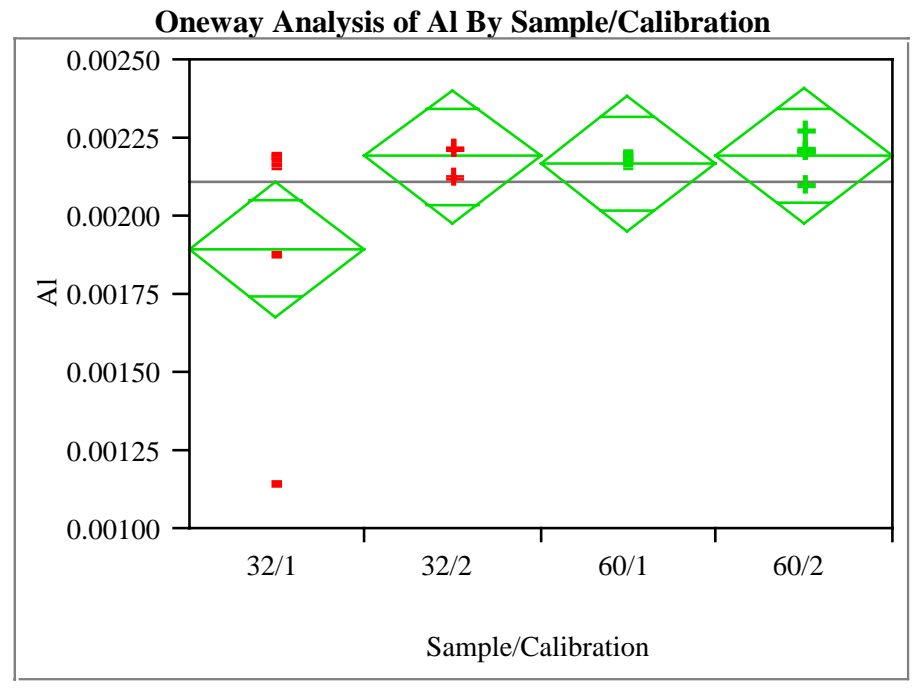

Oneway Analysis of Na By Sample/Calibration




WSRC-TR-2001-00565

Revision 0

Page 79 of 83

November 26, 2001

\section{Appendix (continued)}

\section{Exhibit A17. Statistical Analyses of the Al and Na Concentrations (as grams/gram of supernate) in the Supernate by Type of Composite}

Response Al

Whole Model

Actual by Predicted Plot

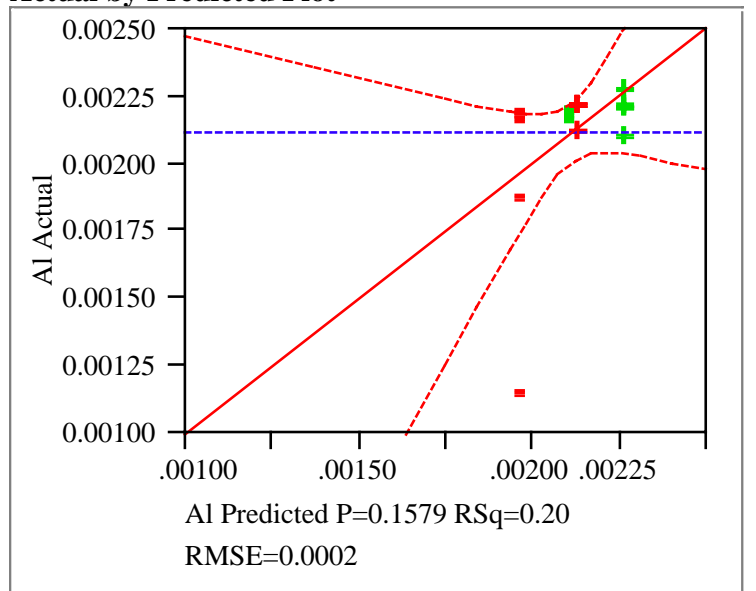

Residual by Predicted Plot

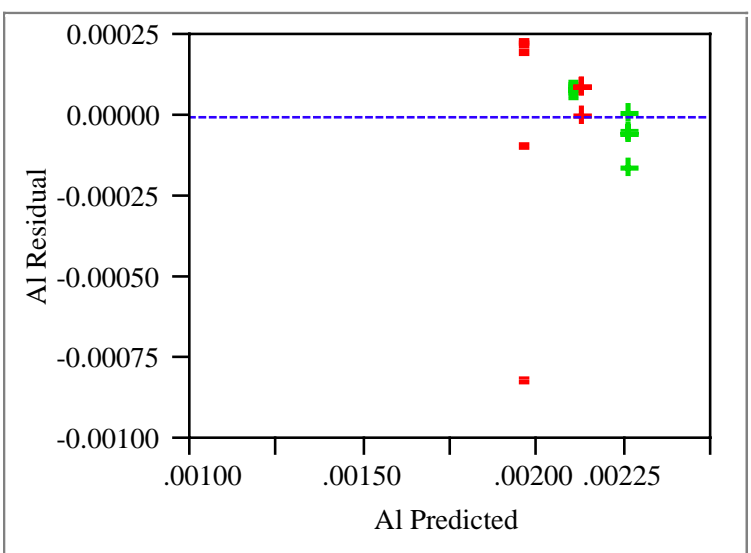

Summary of Fit

R-square is the portion of variation attributed to the model, between 0 and 1. Root Mean Squared Error "RMSE" estimates the standard deviation of the residual.

RSquare

0.195177

RSquare Adj

0.100492

Root Mean Square Error $\quad 0.000233$

Mean of Response $\quad 0.002114$

Observations (or Sum Wgts) 20

Analysis of Variance

The test that the whole model fits better than a simple mean, i.e. testing that all the parameters are zero except the intercept

Source DF Sum of Squares Mean Square F Ratio

$\begin{array}{lllll}\text { Model } & 2 & 0.00000022 & 0.0000001 & 2.0613\end{array}$

$\begin{array}{lllll}\text { Error } & 17 & 0.00000092 & 5.4145 \mathrm{e}-8 \quad \text { Prob }>\text { F }\end{array}$

$\begin{array}{llll}\text { C. Total } & 19 & 0.00000114 & 0.1579\end{array}$

Lack Of Fit

Using replicated points as the part of residual error that does not depend on the form of the model so that you can test for the adequacy of the form of the model.

$\begin{array}{lrrrr}\text { Source } & \text { DF } & \text { Sum of Squares } & \text { Mean Square } & \text { F Ratio } \\ \text { Lack Of Fit } & 1 & 0.00000009 & 8.978 \mathrm{e}-8 & 1.7293 \\ \text { Pure Error } & 16 & 0.00000083 & 5.1917 \mathrm{e}-8 & \text { Prob > F } \\ \text { Total Error } & 17 & 0.00000092 & & 0.2070 \\ & & & & \text { Max RSq } \\ & & & & 0.2737\end{array}$

Parameter Estimates

Term

Intercept

Estimate Std Error t Ratio Prob $>|\mathbf{t}|$

Sample Type[32] $\quad-0.000069 \quad 0.000052 \quad-1.33 \quad 0.2023$

Calibration[1] $\quad-0.00008 \quad 0.000052 \quad-1.54 \quad 0.1426$

Effect Tests

Source

Sample Type

Npa

Calibration

$\begin{array}{rrrrr}\text { Darm } & \text { DF } & \text { Sum of Squares } & \text { F Ratio } & \text { Prob > F } \\ 1 & 1 & 0.0000001 & 1.7586 & 0.2023 \\ 1 & 1 & 0.00000013 & 2.3640 & 0.1426\end{array}$


WSRC-TR-2001-00565

Revision 0

Page 80 of 83

November 26, 2001

\section{Appendix (continued)}

\section{Exhibit A17. Statistical Analyses of the Al and Na Concentrations (as grams/gram of supernate) in the Supernate by Type of Composite (continued)}

Response Na

Whole Model

Actual by Predicted Plot

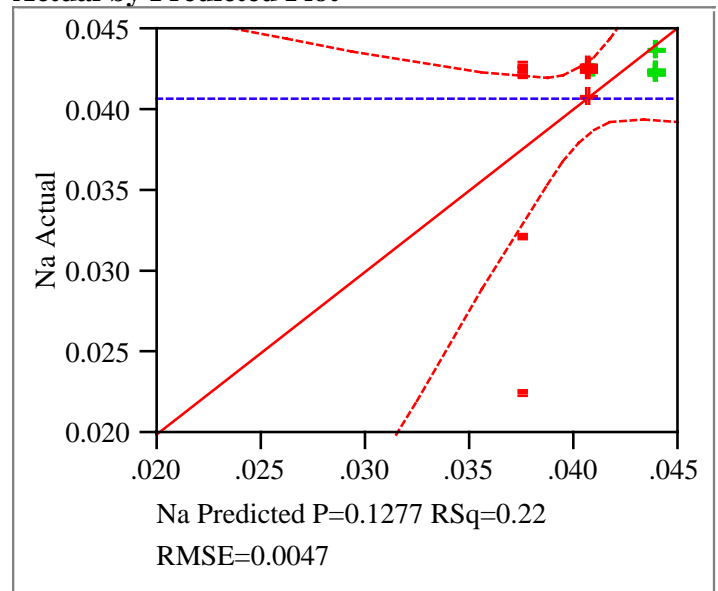

Residual by Predicted Plot

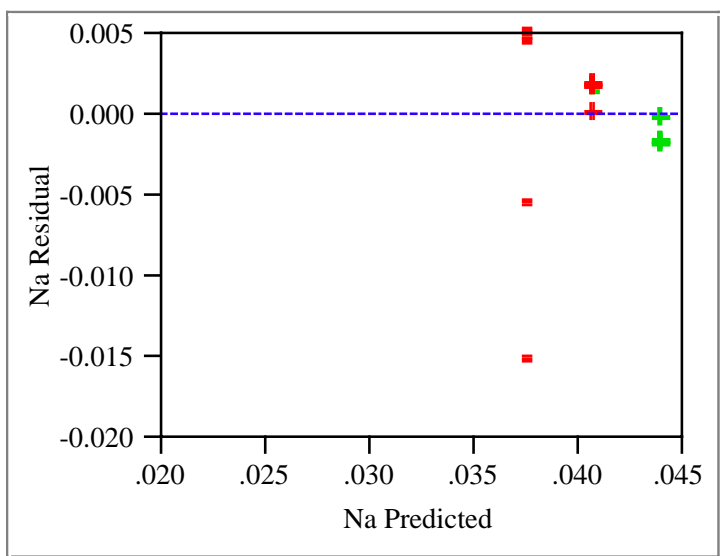

Summary of Fit

R-square is the portion of variation attributed to the model, between 0 and 1. Root Mean Squared Error "RMSE" estimates the standard deviation of the residual.

RSquare

0.215016

RSquare Adj

0.122665

Root Mean Square Error $\quad 0.004676$

Mean of Response $\quad 0.040755$

Observations (or Sum Wgts) 20

Analysis of Variance

The test that the whole model fits better than a simple mean, i.e. testing that all the parameters are zero except the intercept

Source DF Sum of Squares Mean Square F Ratio

$\begin{array}{lllll}\text { Model } & 2 & 0.00010183 & 0.000051 & 2.3282\end{array}$

$\begin{array}{lllll}\text { Error } & 17 & 0.00037174 & 0.000022 & \text { Prob }>\text { F }\end{array}$

$\begin{array}{llll}\text { C. Total } & 19 & 0.00047357 & 0.1277\end{array}$

Lack Of Fit

Using replicated points as the part of residual error that does not depend on the form of the model so that you can test for the adequacy of the form of the model.

$\begin{array}{lrrrr}\text { Source } & \text { DF } & \text { Sum of Squares } & \text { Mean Square } & \text { F Ratio } \\ \text { Lack Of Fit } & 1 & 0.00004176 & 0.000042 & 2.0248 \\ \text { Pure Error } & 16 & 0.00032998 & 0.000021 & \text { Prob > F } \\ \text { Total Error } & 17 & 0.00037174 & & 0.1739 \\ & & & & \text { Max RSq } \\ & & & & 0.3032\end{array}$

Parameter Estimates

Term

Estimate Std Error t Ratio Prob $>|\mathbf{t}|$

Intercept 0.040755

0.001046

$\begin{array}{ll}-0.001635 & 0.001046\end{array}$

38.98

$<.0001$

Sample Type[32]

Calibration[1]

Effect Tests

Source

$-0.001555$

0.001046

$-1.49$

0.1553

Sample Type

Npar

DF Sum of Squares

Calibration

$\begin{array}{rrrrr}\text { Darm } & \text { DF } & \text { Sum of Squares } & \text { F Ratio } & \text { Prob > F } \\ 1 & 1 & 0.00005346 & 2.4449 & 0.1363 \\ 1 & 1 & 0.00004836 & 2.2115 & 0.1553\end{array}$


WSRC-TR-2001-00565

Revision 0

Page 81 of 83

November 26, 2001

\section{Appendix (continued)}

Exhibit A18. Sensitivity of Statistical Analyses of the Al and Na Concentrations (as grams/gram of supernate) in the Supernate to

$5 \%$ Differences Due to Type of Composite

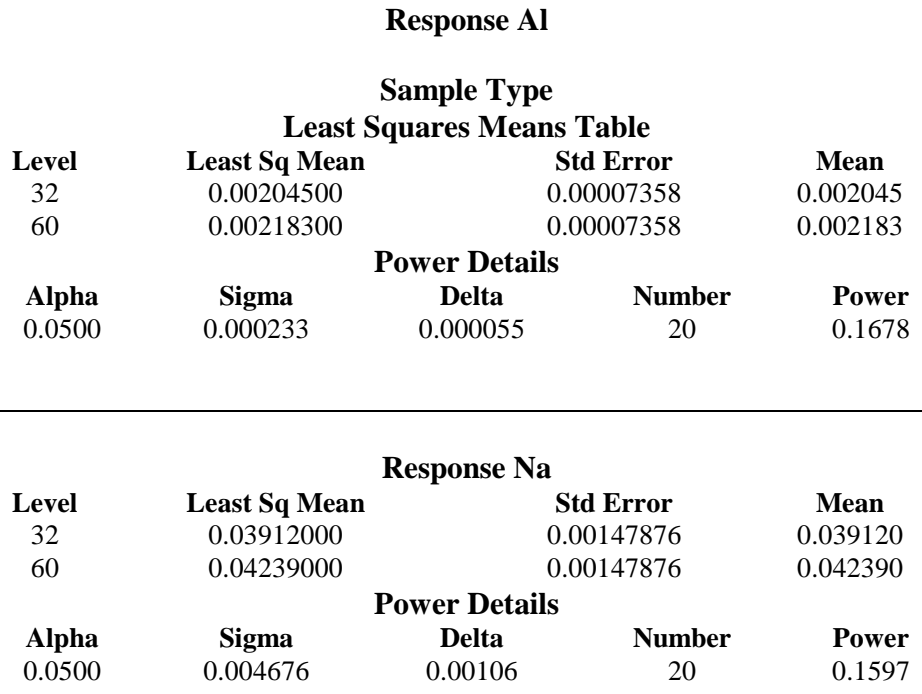


WSRC-TR-2001-00565

Revision 0

Page 82 of 83

November 26, 2001

\section{Appendix (continued)}

\section{Exhibit A19. Statistical Analyses of the Screened Al and Na Concentrations (as grams/gram of supernate) in the Supernate by Type of Composite}

Response Al

\section{LIMS Number Removed From this Analysis: 300166351 and 300166354.}

Actual by Predicted Plot

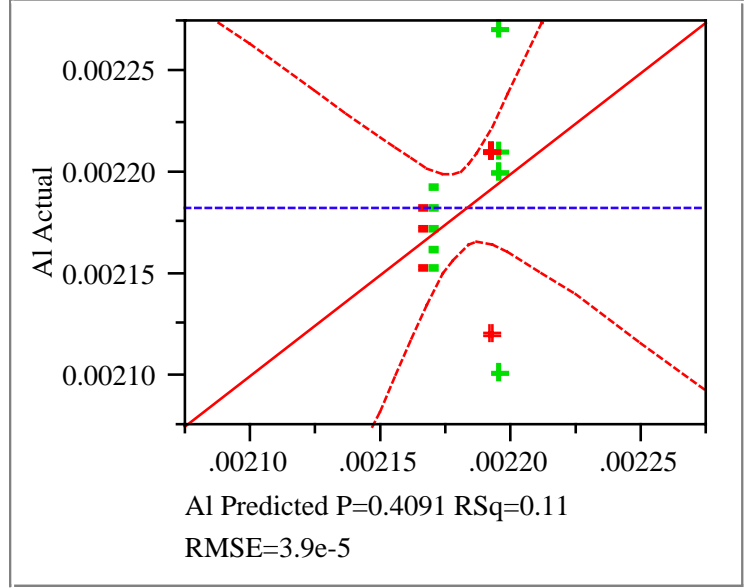

\section{Summary of Fit}

RSquare

RSquare Adj

Root Mean Square Error

Mean of Response

Observations (or Sum Wgts)

0.112339

$-0.00602$

0.000039

0.002183

Analysis of Variance

The test that the whole model fits better than a simple mean, i.e. testing that all the parameters are zero except the intercept

Source DF Sum of Squares Mean Square F Ratio

$\begin{array}{lllll}\text { Error } & 15 & 0.00000002 & 1.5245 \mathrm{e}-9 & \text { Prob > F }\end{array}$

$\begin{array}{llll}\text { C. Total } & 17 & 0.00000003 & 0.4091\end{array}$

Lack Of Fit

Source

Lack Of Fit

DF Sum of Squares

$4.7619 \mathrm{e}-13$

0.00000002

0.00000002

$$
\begin{array}{rr}
\text { Mean Square } & \text { F Ratio } \\
4.762 \mathrm{e}-13 & 0.0003 \\
1.6333 \mathrm{e}-9 & \text { Prob }>\text { F } \\
& 0.9866 \\
& \text { Max RSq } \\
& 0.1124
\end{array}
$$

Parameter Estimates

Term

Intercept

Sample Type[32]

Calibration[1]

Effect Tests

Source

Nparm DF

Estimate Std Error t Ratio Prob $>|t|$ $\begin{array}{lllll}0.0021811 & 0.000009 & 233.69 & <.0001\end{array}$ $\begin{array}{llll}-0.000002 & 0.000009 & -0.20 & 0.8450\end{array}$ $\begin{array}{llll}-0.000013 & 0.000009 & -1.38 & 0.1886\end{array}$

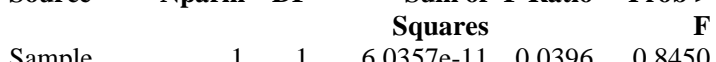

Type

Calibration
Residual by Predicted Plot

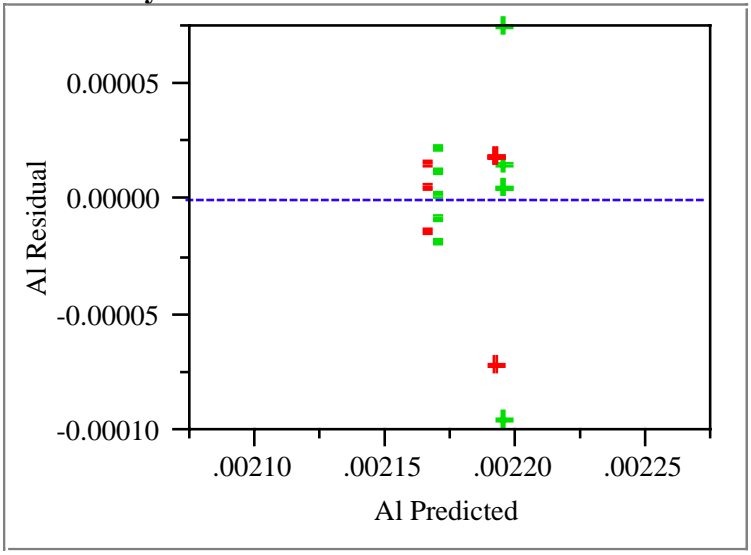

Sample Type

Least Squares Means Table

Level Least Sq Mean Std Error Mean

$\begin{array}{llll}32 & 0.00217929 & 0.00001400 & 0.002182\end{array}$

$\begin{array}{lllll}60 & 0.00218300 & 0.00001235 & 0.002183\end{array}$ 
WSRC-TR-2001-00565

Revision 0

Page 83 of 83

November 26, 2001

\section{Appendix (continued)}

\section{Exhibit A19. Statistical Analyses of the Screened Al and Na Concentrations (as grams/gram of supernate) in the Supernate by Type of Composite (continued)}

Response: Na

\section{LIMS Number Removed From this Analysis: 300166351 and 300166354.}

Whole Model

Actual by Predicted Plot

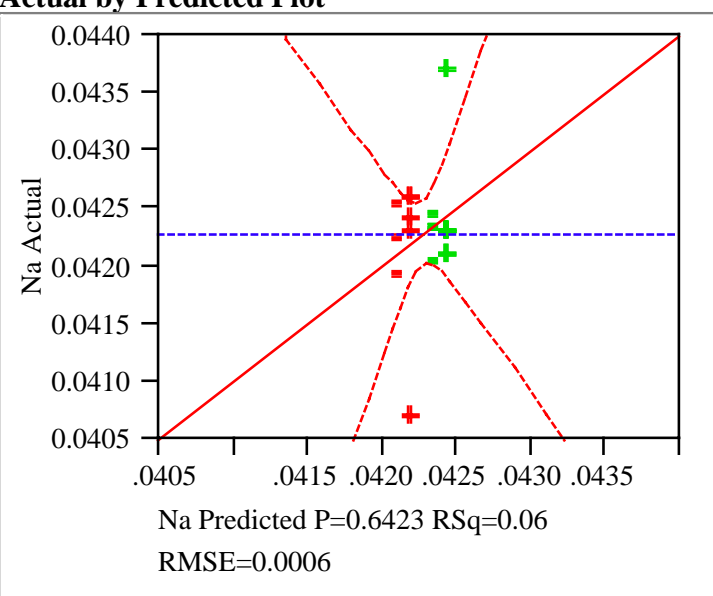

Summary of Fit

RSquare $\quad 0.057311$

RSquare Adj $\quad-0.06838$

Root Mean Square Error $\quad 0.000566$

Mean of Response $\quad 0.042283$

Observations (or Sum Wgts) 18

Analysis of Variance

$\begin{array}{lrrrr}\text { Source } & \text { DF } & \text { Sum of Squares } & \text { Mean Square } & \text { F Ratio } \\ \text { Model } & 2 & 0.00000029 & 0.0000001 & 0.4560\end{array}$

$\begin{array}{lrrrr}\text { Error } & 15 & 0.00000481 & 0.0000003 & \text { Prob }>\text { F }\end{array}$

$\begin{array}{llll}\text { C. Total } & 17 & 0.0000051 & 0.6423\end{array}$

Lack Of Fit

Source DF Sum of Squares

$\begin{array}{lrr}\text { Lack Of Fit } & 1 & 0.0000001\end{array}$

Pure Error $\quad 14 \quad 0.00000472$

Total Error $\quad 15 \quad 0.00000481$

$$
\begin{array}{rr}
\text { Mean Square } & \text { F Ratio } \\
9.6429 \mathrm{e}-8 & 0.2863 \\
0.0000003 & \text { Prob > F } \\
& 0.6010 \\
\text { Max RSq } \\
0.0762
\end{array}
$$

Parameter Estimates

Term

Intercept

Sample Type[32]

Calibration[1]

Effect Tests

Source

Sample Type

Calibration
Npar

$$
\begin{array}{rrrr}
\text { Estimate } & \text { Std Error } & \text { t Ratio } & \text { Prob }>|\mathbf{t}| \\
0.0422643 & 0.000135 & 312.14 & <.0001 \\
-0.000126 & 0.000135 & -0.93 & 0.3679 \\
-0.000046 & 0.000135 & -0.34 & 0.7403
\end{array}
$$

Residual by Predicted Plot



Sample Type

Least Squares Means Table

$\begin{array}{lrrr}\text { Level } & \text { Least Sq Mean } & \text { Std Error } & \text { Mean } \\ 32 & 0.04213857 & 0.00020310 & 0.042150 \\ 60 & 0.04239000 & 0.00017912 & 0.042390\end{array}$

